\title{
Crane-operated warehouses: Integrating location assignment and crane scheduling
}

\author{
Sam Heshmati ${ }^{\mathrm{a}, \mathrm{b}}$, Túlio A. M. Toffolo ${ }^{\mathrm{a}, \mathrm{c}}$, Wim Vancroonenburg ${ }^{\mathrm{a}, \mathrm{d}, *}$, Greet Vanden Berghe ${ }^{\mathrm{a}}$ \\ ${ }^{a} K U$ Leuven, Department of Computer Science, CODeS - Belgium \\ ${ }^{b}$ University of Porto, Faculty of Engineering, INESC TEC - Portugal \\ ${ }^{c}$ Federal University of Ouro Preto, Department of Computing - Brazil \\ ${ }^{d}$ Research Foundation Flanders - FWO Vlaanderen
}

\begin{abstract}
*Corresponding author:
Wim Vancroonenburg, Ph.D. - wim.vancroonenburg@cs.kuleuven.be Ghent Technology Campus, Gebroeders De Smetstraat 1, 9000 Gent, Belgium.
\end{abstract}

\begin{abstract}
Author notes
Wim Vancroonenburg is a postdoctoral researcher funded by Research Foundation Flanders FWO Vlaanderen. Editorial consultation provided by Luke Connolly (KU Leuven).

Data instances + validator available at : https://bitbucket.org/Sam-Hes/cwsp/
\end{abstract}




\title{
Crane-operated warehouses: Integrating location assignment and crane scheduling
}

\begin{abstract}
Crane-operated warehouses constitute an essential asset for the many industries which must temporarily store products on their way from manufacturers to consumers. Such warehouses are a practical necessity rather than an explicitly desired service and they introduce significant operational costs which should be minimized. The problem addressed by the current paper, the Crane-operated Warehouse Scheduling Problem (CWSP), concerns the location assignment of input products and the scheduling of cranes for product movement in such warehouses. Several constraints are associated with the problem, for example certain products should not be stored close to each other (due perhaps to a difference in temperature or aroma) and cranes must respect operational safety distances between each other in order to prevent dangerous collisions. The present paper explores a novel methodology which combines these two decisions - location assignment and crane scheduling - instead of solving them sequentially. In addition to mathematical formulations for location assignment and crane scheduling, both an integrated mathematical formulation and a fast heuristic are presented for the CWSP. The quality of the mathematical formulation and the heuristic are compared against the conventional sequential approaches. Experimentation upon an extensive range of instances show significantly improved results are attainable when integrating location assignment and crane scheduling, despite some (expected) increase in computational time.
\end{abstract}

Keywords: Crane-operated warehouse scheduling, Crane scheduling, Crane interference, Location assignment

\section{Introduction}

Warehouses constitute a form of infrastructure commonly employed by manufacturers, wholesalers and retailers to store goods not only during the production process but also during their distribution. Warehouse efficiency therefore plays a crucial role in global economy. Their efficiency enhances the capacity of supply chains, providing significant economic and service benefits to both businesses and end users. Reducing storage and handling costs, increasing warehouse capacity and improving the timeliness of deliveries are essential to further sustaining and strengthening supply chains. The present study focuses on the first aspect, in particular in Crane-operated warehouses.

The term 'Craned-operated warehouse' refers to a type of warehouse or storage area which employs any type of overhead crane such as rubber-tired gantry cranes (RTGCs) or rail mounted gantry cranes (RMGCs). Overhead cranes are commonly used in industrial warehouses where the stored products are rather heavy and large-sized in nature (examples of which include steel coils, large pallets of goods, ...) or in container terminals where containers are temporarily stored in stacks before being transferred to their next destinations. The Crane-operated Warehouse Scheduling Problem (CWSP) studied throughout this paper concerns the optimization of both the products' storage location and the crane operations which are necessary to do so in warehouses 
which employ such overhead cranes. A warehouse typically consists of a set of input and output points which are located in the periphery of a storage area. Products are stored subject to a range of operational constraints and a set of cranes are employed for handling operations. In many cases, cranes cannot overtake each other (such as when they are operating on the same pair of rails), thereby necessitating proper safety measures to avoid collisions. The CWSP as such is composed of two constituent optimization problems, namely:

i) The Location Assignment Problem (LAP): assigning the storage locations to incoming products and those which must be relocated within the storage area.

ii) The Crane Scheduling Problem (CSP): scheduling the cranes' operations.

The objective is to minimize both total storage cost and tardiness of crane operations.

The CWSP is conventionally split into the two aforementioned sub-problems - the LAP and CSP - which are solved sequentially. First, the LAP is solved and the resulting storage locations for incoming/relocated products are fixed. Next, the CSP is solved to determine the best schedule for the handling operations. To date, there has been a considerable lack of research which assesses the impact of integrating these two sub-problems.

Container terminals represent one specific real-world application where the CWSP is encountered. Given the continuously increasing volume of containers being handled in terminals worldwide, which places significant pressure on terminals' infrastructure and operations, it is unsurprising that there exists a vast body of container terminal literature relevant to the problem.

The majority of studies related to the LAP involve optimization problems in container terminals such as the re-handling problem (Jovanovic and Voß, 2014, $\mathrm{Ku}$ and Arthanari, 2016) and the container stacking problem (Zhang et al. $\mid 2014$; Gharehgozli et al., 2014). The re-handling problem concerns removing containers from stacks to enable a given set of container retrievals where the objective is to minimize the number of moves. Studies addressing the container stacking problem mostly focus on minimizing reshuffling, namely those unproductive moves required to gain access to a desired container which is blocked (Chen and Lu, 2012; Boysen and Emde, 2016). Other objectives include minimizing travelling distance, wasted space, or estimated retrieval cost (Park et al., 2011).

The objective function of the LAP in the present study derives itself directly from operational practices found in production industries and differs from objective functions found in references related to container terminals. It includes cost terms related to storing a product in a specific location and others related to storing certain products adjacent to one another. The former cost terms are used to model the retrieval costs (specified as a distance from an output point), while the latter model operational constraints of production industries which seek to avoid storing certain products in close proximity. For instance, companies may wish to avoid storing aromatic products next to each other or may require hot products to be stored away from those which have already cooled down. While companies may often disallow such neighbouring location assignments altogether, in situations of high storage occupancy it may not always be feasible to do so. Addressing such situations as soft constraints, penalized as costs in the objective function, enables the necessary modelling flexibility and avoids infeasibility.

Many studies address the CSP independent from the LAP, considering the LAP's solution as a fixed input. The most relevant references to the present study are those focused on scheduling multiple cranes. For single crane scheduling, interested readers are referred to the survey by Boysen 
and Stephan (2016). Due to the increasing necessity to accelerate handling operations in warehouses, many recent papers have focused on scheduling multiple cranes operating simultaneously within the same storage area. Dorndorf and Schneider (2010) studied a container yard in which a pair of cranes operates on the same rails with another larger crane operating above them (cross-over crane) on its own pair of rails. Each of the two smaller cranes has its own distinct working area to avoid collisions. Given independent and mutually-exclusive working areas and the presence of a separate cross-over crane, crane interference does not pose a problem in such yards. By contrast, Li et al. (2009) considered a container terminal which employs multiple cranes that may interfere with one another. They proposed a discrete-time MIP model for the problem and a heuristic to solve it. Li et al. (2012) extended Li et al. (2009)'s work by proposing a continuous time MIP model capable of handling instances with a higher number of storage and retrieval requests. $\mathrm{Wu}$ et al. (2015) also considered a container terminal with multiple cranes, as Li et al. (2009, 2012), and proposed a polynomial time heuristic to solve their optimization problem. These studies are particularly interesting with regard to how they model the scheduling of multiple cranes operating in storage areas with inter-crane interference. However a noteworthy and significant difference with respect to the present work lies in how within all the aforementioned studies containers are delivered. This means cranes remain static at the stacking piles and do not move during handling operations. Consequently, the duration of all operations can be assumed to be equal. This simplifies the problem modeling by enforcing equal time durations for all operations. In a general setting, however, input and output may occur anywhere around the storage area and cranes move over that storage area while handling products. Gharehgozli et al. (2017) investigated a set of rules and their influence on the effect of temporary locations in a so-called handshake area which facilitates container handover between cranes. The paper presented some managerial insights on the size, location, and number of such handshake areas.

Gharehgozli et al. (2015) attempted to integrate location assignment and crane scheduling problems in a container terminal, wherein the water-side crane performs all requests which must be stacked or retrieved from the water-side, and land-side operations are carried out similarly by a land-side crane. However a significant limitation to their model is that storage and retrieval requests are already assigned to cranes in advance. Moreover, the model was designed for only one land-side and one water-side crane, and thus cannot accommodate cases with more than two cranes, or cases where both cranes may handle requests from anywhere throughout the storage area.

In practice, warehouse managers are becoming increasingly aware that warehouse efficiency may be bolstered by exploiting an integrated optimization approach, where location assignment and crane scheduling decisions are simultaneously taken into consideration and jointly optimized (Darvish and Coelho, 2018). The literature is however lacking studies that investigate this. The present research, therefore, focuses on this integrated approach of handling the CWSP. It provides a general setting which may be easily adapted to other warehouses, land-side container terminals or any other industry employing multiple gantry cranes for product handling.

Mathematical formulations and heuristics are developed and tested upon a set of instances which are randomly-generated using probability distributions and insights extracted from a relevant industrial case. Results are compared against those obtained with a heuristic based on the dispatching rules and manual strategies employed in practice. The findings from this computational study reveal the significant benefits of combining the LAP and CSP when solving the CWSP.

The remainder of the paper is structured as follows. Section 2 provides a detailed problem 
definition of the CWSP. Section 3 presents mathematical formulations for the LAP and the CSP, and also formulates the CWSP by means of a continuous-time mixed integer programming model which considers realistic constraints. Section 4 presents a heuristic algorithm for solving the LAP, CSP and CWSP. Computational experiments and a comparative algorithmic performance analysis are detailed throughout Section 5. Finally, Section 6 summarizes the paper's primary findings and discusses possible future research directions.

\section{Problem definition}

Throughout this study, a crane-operated warehouse is considered which consists of a storage area within which products are placed. The storage area is composed of locations, with each location storing at most one product. A set of special locations representing input/output (I/O) points around the storage area is defined where input requests originate and output requests must be delivered. Each I/O point either originates input or collects output requests which must be processed by their due time.

Each request consists of a product that must be moved. Requests are divided into two sets:

- Input requests $\left(\mathcal{R}^{I}\right)$ : requests which require location assignment. $\mathcal{R}^{I}$ consists of requests for products at an input point requiring transfer to the yard or products that must be moved within the yard to enable cranes to access locations, located beneath them, associated with output requests;

- Output requests $\left(\mathcal{R}^{O}\right)$ : requests consisting of products within the yard requiring transfer to an output point.

Set $\mathcal{R}$ represents the union of the two sets: $\mathcal{R}=\mathcal{R}^{I} \cup \mathcal{R}^{O}$. A release time and due time are associated with each request, defining when the product is available for transfer and when it is due to be transferred.

The set of available locations $L$ consists of locations that are already free or will become free during the scheduling horizon when their stored product has been moved. This includes the origin location of the output requests and those requests which move products inside the yard. $L$ excludes locations which store products that will not be moved during the scheduling horizon. Following convention, the storage area length is mapped to a horizontal coordinate axis. A horizontal coordinate $h_{l}$ is associated with each individual location $l \in L$. The horizontal coordinates in the yard are ordered from left to right. A product may be stored in a location above ground level, stacked on another product. Therefore, in addition to its horizontal and lateral coordinates, a location $l$ is also defined by its level above the ground. To be able to store a product in a location above ground level, all locations beneath the product must be occupied by other products. Cranes are employed to execute input and output requests. A set of available, identical cranes $\mathcal{C}$ is defined, each being capable of handling one request at a time. Cranes are mounted on a pair of rails along the horizontal axis, and are ordered and indexed from left to right in the storage area. Additionally, cranes have no predefined working areas, the only restriction being that they cannot cross and that a safety distance must be respected between neighbouring cranes while moving throughout the storage area. This study assumes that cranes can reach all locations.

Figure 1 illustrates a top-view of a crane-operated storage area in which the gray border represents the input/output points. Three cranes are ordered from left to right and operate across the 
storage area. Note that the safety distance must be respected and therefore, cranes cannot pass over each other.

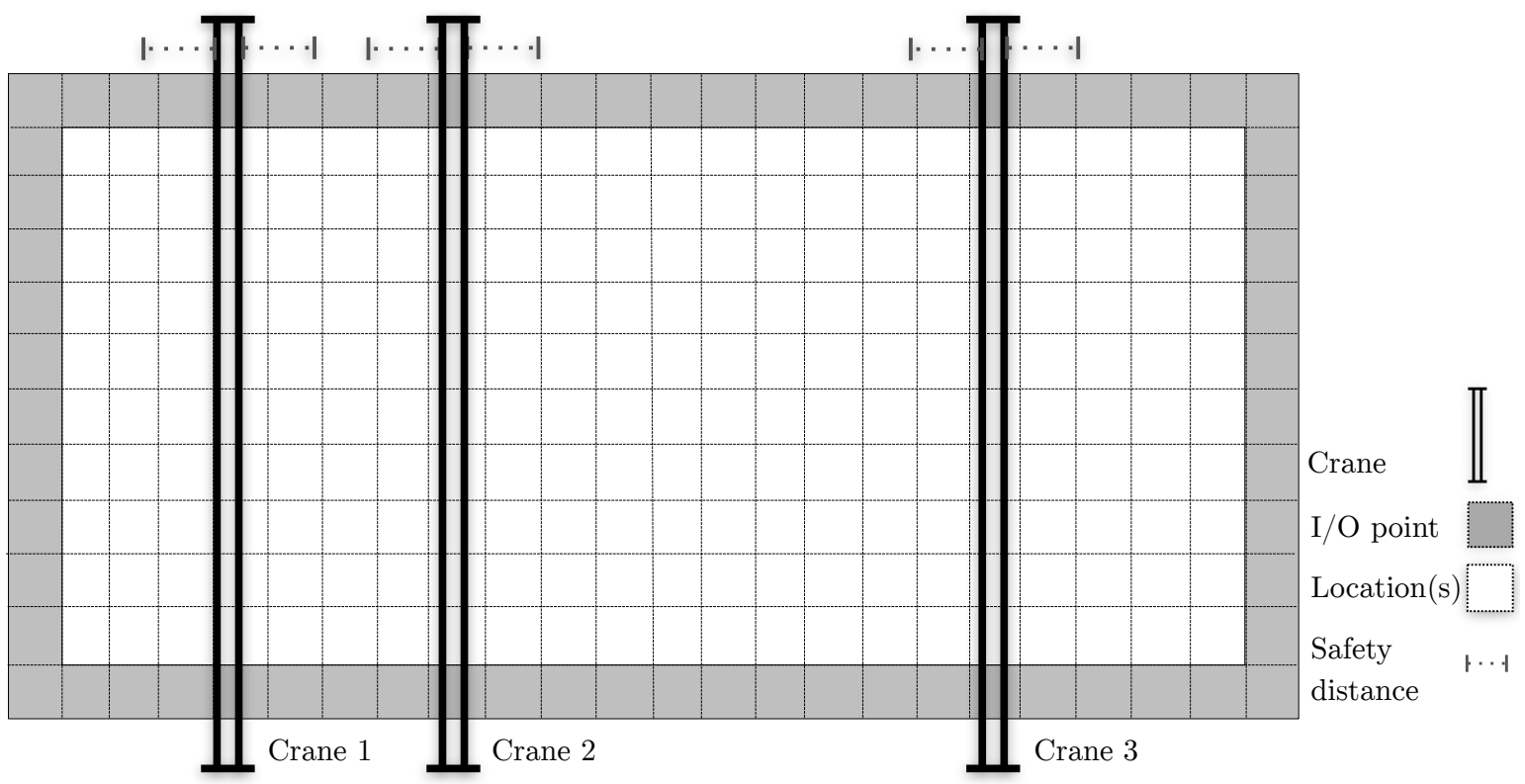

Figure 1: Top view of a warehouse employing three cranes.

The CWSP consists of two optimization problems, the LAP and the CSP. The LAP's objective is to minimize the total storage cost of input requests. The storage cost for a product is defined in terms of an assignment in the neighbourhood of other products in the storage area. The total storage cost includes the cost, summed over all input requests, of assigning an input request to a location in the storage area (pre-calculated and corresponding with the distance to neighbouring products which will not move during the scheduling horizon) and the cost of assigning two input requests in neighbouring locations.

The CSP consists of deciding when and by which crane each request will be executed, while respecting precedence constraints and constraints concerning safety distances. Precedence constraints may be predetermined or introduced during location assignment. Predetermined precedence constraints follow from when a product is stacked on top of a product associated with an output request, the top product must be removed first after which the output request may be executed. Precedence constraints introduced during location assignment follow from assigning an input request to the location of a request originated within the yard (either input or output) or assigning two input requests on top of each other. The objective is to minimize total tardiness of all requests. A request's tardiness equals the difference between its completion time and due time if positive, or zero otherwise.

The combined problem of solving both the LAP and CSP simultaneously is referred to as the CWSP. The objective of the CWSP is to minimize the weighted linear expression presented in Equation (1), where $\alpha$ and $\beta$ are weights defining the relative importance of the terms, while $E_{L A}$ and $E_{C S}$ correspond to total storage cost and total tardiness, respectively.

$$
\text { Total cost }=\alpha \cdot E_{L A}+\beta \cdot E_{C S}
$$




\section{Mathematical formulation}

This section presents mathematical formulations for the LAP (Section 3.1) and CSP (Section 3.2), followed by a formulation for the CWSP (Section 3.3) which considers the LAP and CSP simultaneously.

\subsection{Location assignment problem}

Formulation $\mathcal{F}_{L A}$ concerns the assignment of destination locations to input requests. Table 1 summarizes the notation employed for the LAP formulation.

$$
\mathcal{F}_{L A} \begin{cases}\min \sum_{i \in \mathcal{R}^{I}} \sum_{l \in L} \gamma_{i l} x_{i l}+\sum_{i \in \mathcal{R}^{I}} \sum_{j \in \mathcal{R}^{I}} \omega_{i j} z_{i j} & \\ \text { s.t. } \sum_{l \in L: l \neq b_{i}} x_{i l}=1 & \forall i \in \mathcal{R}^{I} \\ \sum_{i \in \mathcal{R}^{I}} x_{i l} \leq 1 & \forall l \in L \\ x_{i l} \leq \sum_{j \in \mathcal{R}^{I}} x_{j k} & \forall i \in \mathcal{R}^{I}, l \in L, k \in U_{l} \\ x_{i l}+x_{j k} \leq 1+z_{i j} & \forall i, j \in \mathcal{R}^{I}, l \in L, k \in N_{l} \\ x_{i l} \in\{0,1\} & \forall i \in \mathcal{R}^{I}, l \in L \\ z_{i j} \in\{0,1\} & \forall i, j \in \mathcal{R}^{I},\end{cases}
$$

Objective function (2) minimizes the total storage cost. The storage cost is divided into two parts: the cost of assigning request $i$ to location $l$ and the cost of assigning requests $i$ and $j \in \mathcal{R}^{I}$ in each other's neighbourhood, denoted by $\omega_{i j}$ and $\gamma_{i l}$ respectively. Constraints (3) and (4) are classic assignment constraints ensuring exactly one location is assigned to each input request and that each location receives, at most, a single request, respectively. Constraints (3) also prevent assigning input requests to their origin locations. If request $i$ represents a product that must be moved within the yard to access a product below, other input requests may use $b_{i}$ as their destination, after the product below $b_{i}$ has been moved. Constraints (5) force all available locations underneath location $l \in L$ to have an input request assigned, thus ensuring that no product is stacked atop an empty location. It is sufficient to assure there is an input request assigned to each available location $k \in U_{l}$, since the products not associated with any requests will not be moved during the scheduling horizon. Constraints (6) set the value of $z_{i j}$ to 1 if requests $i$ and $j$ are placed in neighbouring locations and 0 otherwise. Constraints (7) and (8) state variables $x_{i l}$ and $z_{i j}$ are binary.

Following the LAP, precedence constraints may be implied when input requests are assigned to the origin locations of output requests or when two input requests are assigned to locations where one is on top of the other. The outcome of the LAP (destination location for input requests) along with the set of precedence requests is the input for the CSP.

\subsection{Crane scheduling problem}

Formulation $\mathcal{F}_{C S}$ models the CSP which considers the crane assignment for requests and the sequencing of requests per crane. $\mathcal{F}_{C S}$ implements various realistic operational constraints such as 
Table 1: Notations for the LAP formulation

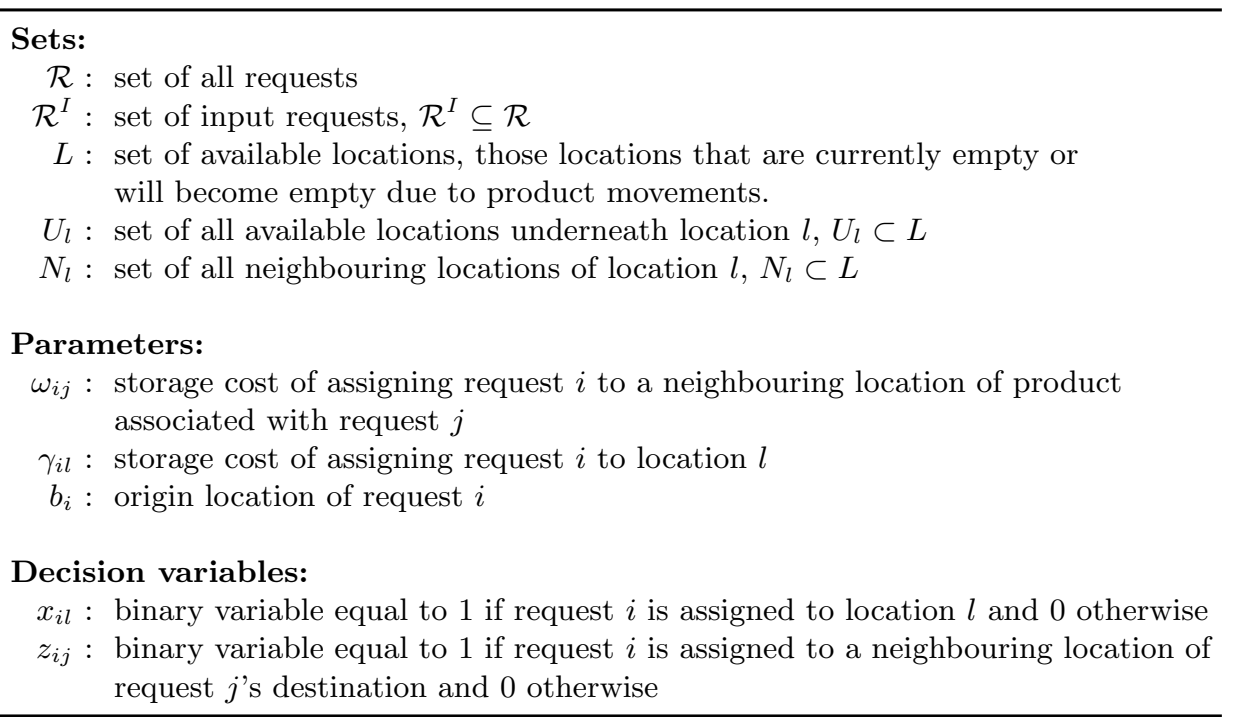

multiple cranes working simultaneously in the storage area and precedence constraints. Note that each crane can traverse the entire storage area provided safety distances between all cranes are respected. This means that a crane may move beyond the storage area boundary to provide space for another crane to access storage location at or close to the area's perimeter.

The continuous-time formulation for the CSP presented in this paper was inspired by Li et al. (2012), who showed that for the CSP with multiple cranes this formulation significantly reduced the model's size and enabled larger instances to be solved compared to a discrete-time formulation for the same problem. Recall from Section 1 that Li et al. (2012) considered the CSP in a container terminal where containers were brought directly in front of the stacking pile. As a consequence, cranes do not move along the rails when moving a product. They instead move products laterally (along the crane beam). However, in a general setting of crane-operated warehouses the cranes move along the storage area to reach the respective input/output point during their operations. Conflicting requests and variable operation durations are consequently inevitable. The model presented in the following section accounts for this additional complexity. Table 2 summarizes the notation employed to formulate the CSP.

The constraints of $\mathcal{F}_{C S}$ are organised into three categories: $(i)$ request assignments for cranes, (ii) handling conflicting requests and, finally, (iii) setting the requests' starting times.

\section{(i) Request assignments for cranes}

Constraints (9) ensure that exactly one crane is assigned to each request. Constraints (10) and (11) determine the value of variable $n_{i j}$ which must be 1 if request $i$ finishes before the starting time of request $j$ and 0 otherwise. 
Table 2: Notations for the CSP formulation

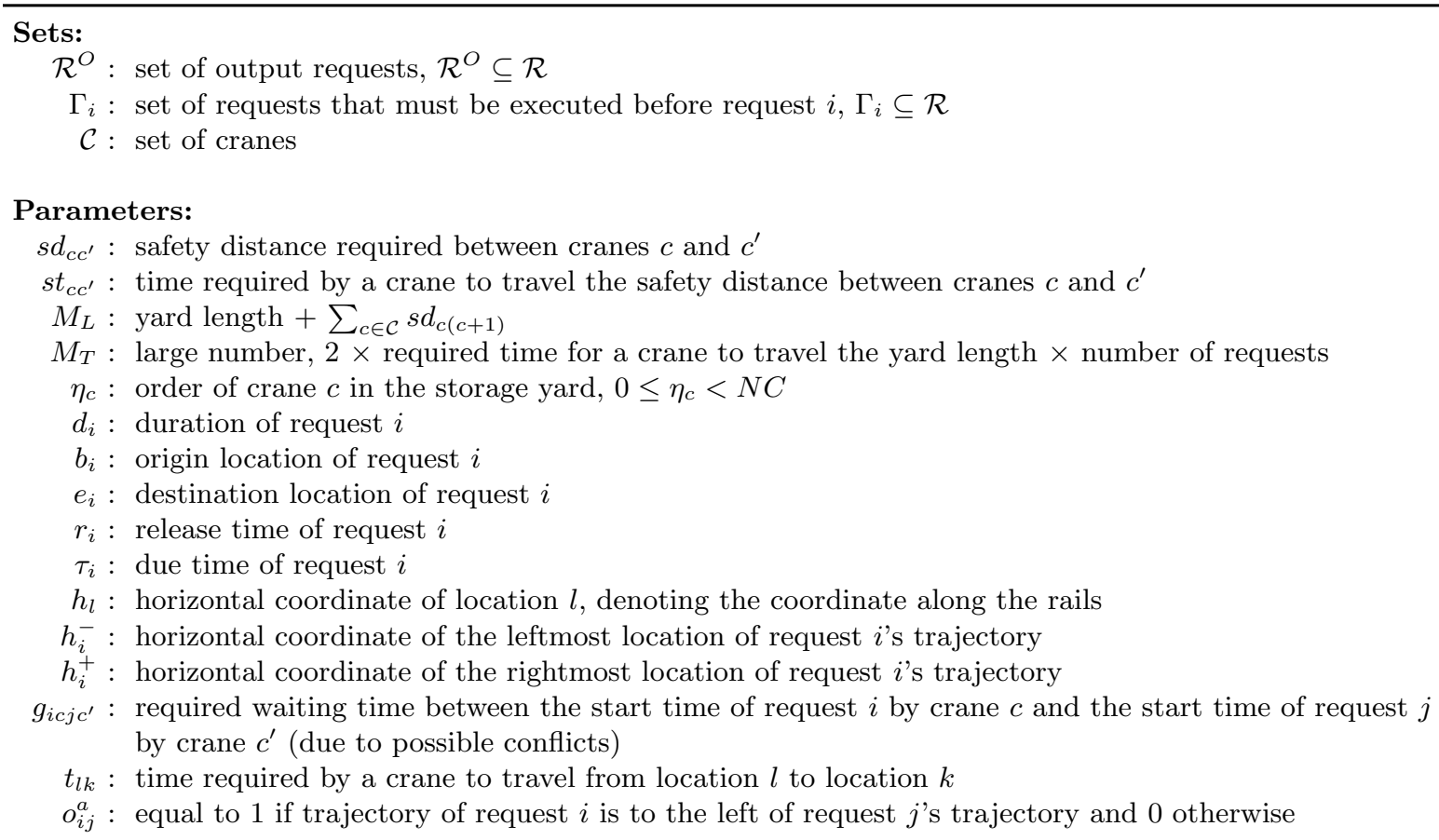

\section{Decision variables:}

$y_{i c}$ : binary variable equal to 1 if request $i$ is handled by crane $c$ and 0 otherwise

$s_{i}$ : continuous variable indicating the start time of request $i$

$\delta_{i}$ : continuous variable indicating the tardiness of request $i$

\section{Auxiliary variables:}

$n_{i j}$ : binary variable equal to 1 if request $i$ finishes before the start time of request $j$ and 0 otherwise

$q_{i j}$ : binary variable equal to 1 if request $i$ begins before the start time of request $j$ and 0 otherwise

$o_{i j}$ : binary variable equal to 1 if requests $i$ and $j$ are conflicting and 0 otherwise

$o_{i j}^{b}$ : binary variable equal to 1 if the crane assigned to request $i$ is to the right of the crane assigned to request $j$ and 0 otherwise.

$o_{i j}^{c}$ : binary variable equal to 1 if the distance between $h_{i}^{-}$and $h_{j}^{+}$is less than the safety distance required between cranes handling them and 0 otherwise 


$$
\begin{array}{lll}
\sum_{c \in \mathcal{C}} y_{i c}=1 & & \forall i \in \mathcal{R} \\
s_{i}+d_{i} \geq s_{j}-M_{T} n_{i j} & & \forall i, j \in \mathcal{R}: i \neq j \\
s_{i}+d_{i} \leq s_{j}+\left(1-n_{i j}\right) M_{T} & & \forall i, j \in \mathcal{R}: i \neq j
\end{array}
$$

Each crane may move only one product at a time. When two requests are scheduled within overlapping times $\left(n_{i j}=n_{j i}=0\right)$, they must be assigned to different cranes. Figure 2 presents two cases involving requests $i$ and $j$ where the horizontal axis represents time $(t)$. In the first case, $s_{j}$ (starting time of $j$ ) is larger than $s_{i}$ and smaller than $s_{i}+d_{i}$ (finishing time of $i$ ), and thus $n_{i j}=n_{j i}=0$ (time overlapping requests). In the second case, request $j$ is executed after request $i$ is finished and, therefore, $n_{i j}=1$ and $n_{j i}=0$ (non-overlapping moves).

$$
n_{i j}=n_{j i}=0
$$

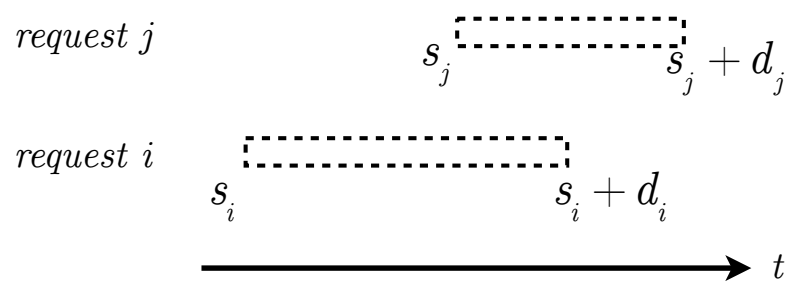

$$
n_{i j}=1, n_{j i}=0
$$

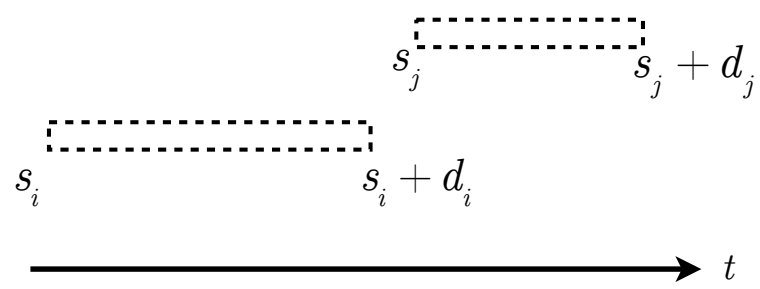

Figure 2: An example of overlapping and non-overlapping requests with respect to time.

Constraints 12 prevent the assignment of time-overlapping requests to the same crane.

$$
y_{i c}+y_{j c} \leq 1+n_{i j}+n_{j i} \quad \forall i, j \in \mathcal{R}: i \neq j, \forall c \in \mathcal{C}
$$

\section{(ii) Handling conflicting requests}

Cranes cannot pass each other and must respect a safety distance to avoid collision. The physical constraints due to non-crossing and safety requirements of cranes pose a significant challenge. If simultaneously executing requests $i$ and $j$ violates the safety distance, then these requests are conflicting and must be scheduled at different times. When this situation occurs, binary auxiliary variable $o_{i j}$ is set to 1 , indicating requests $i$ and $j$ are conflicting.

To assist in identifying conflicting requests, the minimum horizontal coordinate $\left(h_{i}^{-}\right)$and maximum horizontal coordinate $\left(h_{i}^{+}\right)$of a request $i$ are employed and are independent of the requests movement direction. Since the origin and destination locations of requests are given by the location assignment, values $h_{i}^{-}=\min \left(h_{b_{i}}, h_{e_{i}}\right)$ and $h_{i}^{+}=\max \left(h_{b_{i}}, h_{e_{i}}\right)$ are easy to pre-compute.

Requests assigned to different cranes may be conflicting depending on their minimum and maximum horizontal coordinates and on the position of the assigned cranes. Given two requests $i$ and $j$ handled by cranes $c$ and $c^{\prime}$ respectively and a required safety distance $s d_{c c^{\prime}}$, two different situations are possible. The first situation arises when the cranes must pass each other to handle the requests whereas the second situation occurs when there is insufficient space for them to respect the safety distance and handle the requests. 
Figure 3 illustrates trajectories of two requests $i$ and $j$ as well as of cranes $c$ and $c^{\prime}$. Two cases may be considered for these two requests. In the first case where crane $c$ is assigned to request $i$ and crane $c^{\prime}$ to request $j,\left(y_{i c}=y_{j c^{\prime}}=1\right)$, the allocation is such that no conflict occurs. In the second scenario, however, inverting the crane assignments $\left(y_{i c^{\prime}}=y_{j c}=1\right)$ renders the simultaneous handling of requests impossible, given that cranes cannot pass each other therefore, requests $i$ and $j$ are conflicting, and starting times $s_{i}$ and $s_{j}$ must be different.

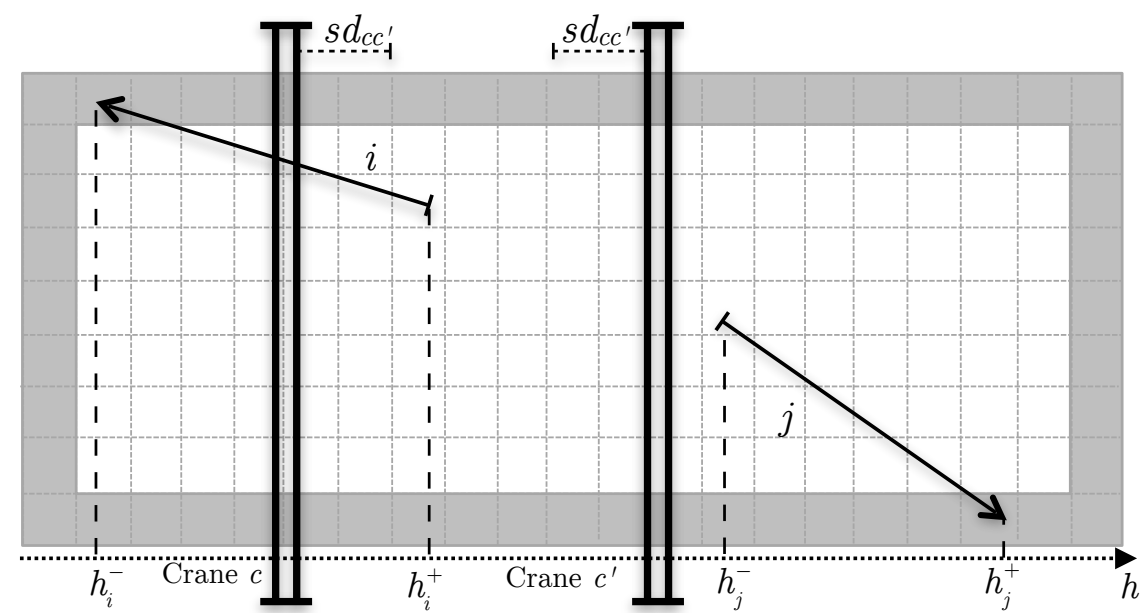

Figure 3: Conflicting requests due to crane assignments.

Parameter $o_{i j}^{a}$ and auxiliary variable $o_{i j}^{b}$ identify conflicting requests due to cranes requiring to pass each other. $o_{i j}^{a}$ indicates whether the trajectory of request $i$ is completely to the left of $j$ 's trajectory, such that $h_{i}^{+}<h_{j}^{-} \Rightarrow o_{i j}^{a}=1$. Constraints (13) are employed to define the values of $o_{i j}^{b}$, which equals 1 if the crane assigned to request $i$ is to the right of the crane assigned to request $j, o_{i j}^{b}=1$.

$$
\sum_{c \in \mathcal{C}} \eta_{c} y_{j c} \geq \sum_{c \in \mathcal{C}} \eta_{c} y_{i c}-|\mathcal{C}| \cdot o_{i j}^{b} \quad \forall i, j \in \mathcal{R}: i \neq j
$$

Whenever both $o_{i j}^{a}$ and $o_{i j}^{b}$ equal one, $i$ 's trajectory is to the left of $j$ 's trajectory while $i$ 's crane is to the right of $j$ 's crane, resulting in a conflict. Constraints (14) force $o_{i j}$ to take a value of 1 whenever $o_{i j}^{a}=o_{i j}^{b}=1$. Likewise, Constraints (15) force $o_{i j}$ to take value 1 whenever $o_{j i}^{a}=o_{j i}^{b}=1$. When request $i$ is conflicting with request $j$, then request $j$ is conflicting with request $i$, implying $o_{i j}=o_{j i}$.

$$
\begin{array}{ll}
o_{i j} \geq o_{i j}^{a}+o_{i j}^{b}-1 & \forall i, j \in \mathcal{R} \\
o_{i j} \geq o_{j i}^{a}+o_{j i}^{b}-1 & \forall i, j \in \mathcal{R}
\end{array}
$$

Another cause of request conflict concerns the safety distance between cranes. Figure 4 illustrates the trajectory of two requests $i$ and $j$ and cranes $c$ and $c^{\prime}$. Requests $i$ and $j$ are conflicting as there is insufficient space for the cranes to begin handling the requests while respecting the safety distance $\left(h_{i}^{-}-h_{j}^{+}<s d_{c c^{\prime}}\right)$. Given the position of the requests' minimum and maximum horizontal 
coordinates, they are conflicting and the cranes cannot begin executing them simultaneously.

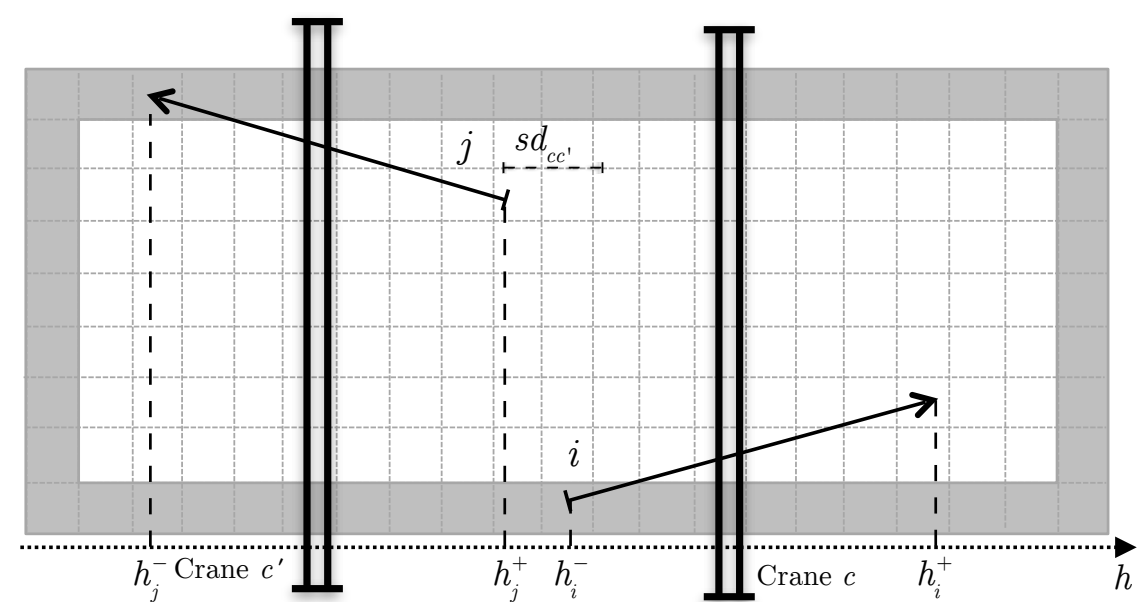

Figure 4: Conflicting requests with overlapping trajectories.

Auxiliary binary variables $o_{i j}^{c}$ are introduced to identify such conflict. Constraints (16) determine the value of $o_{i j}^{c}$. Whenever $i$ and $j$ are handled by cranes $c$ and $c^{\prime}\left(y_{i c}=y_{j c^{\prime}}=1\right.$ or $\left.y_{i c^{\prime}}=y_{j c}=1\right)$, the maximum value for $y_{i c}+y_{i c^{\prime}}+y_{j c}+y_{j c^{\prime}}$ equals two and therefore, Constraints (16) ensure $o_{i j}^{c}$ equals one whenever the minimum horizontal coordinate of $i, h_{i}^{-}$, conflicts with the maximum horizontal coordinate of $j, h_{j}^{+}$, and 0 otherwise $\left(h_{i}^{-}-h_{j}^{+}<s d_{c c^{\prime}} \Rightarrow o_{i j}^{c}=1\right)$.

$$
h_{i}^{-}-h_{j}^{+} \geq s d_{c c^{\prime}}-\left(2-y_{i c}-y_{i c^{\prime}}-y_{j c}-y_{j c^{\prime}}+o_{i j}^{c}\right) M_{L} \quad \forall i, j \in \mathcal{R}: i \neq j, c, c^{\prime} \in \mathcal{C}: c \neq c^{\prime}
$$

Whenever both $o_{i j}^{c}$ and $o_{j i}^{c}$ equal one, a conflict is detected. Constraints (17) force $o_{i j}$ (and $o_{j i}$ ) to take a value of 1 whenever $o_{i j}^{c}=o_{j i}^{c}=1$.

$$
o_{i j} \geq o_{i j}^{c}+o_{j i}^{c}-1 \quad \forall i, j \in \mathcal{R}: i \neq j
$$

(iii) Setting the requests' starting times

A crane can handle one request at a time, therefore, to execute two consecutive requests, a crane requires sufficient time to finish executing the first request and then travel from its destination to the second request's origin.

If two requests $i$ and $j$ are handled by a single crane $c,\left(y_{i c}=y_{j c}=1\right), i$ and $j$ must be handled one at a time $\left(n_{i j}=1\right.$ or $\left.n_{j i}=1\right)$. Assume request $j$ is executed after finishing $i\left(n_{i j}=1\right)$. Constraints (18) ensure starting time $s_{j}$ is greater than or equal to the sum of $i$ 's finishing time $\left(s_{i}+d_{i}\right)$ and the time required by the crane to travel from $i$ 's destination to $j$ 's origin $\left(t_{e_{i} b_{j}}\right)$. Constraints (18) hold when $y_{i c}+y_{j c}+n_{i j}=3$ (requests are handled by a single crane and $j$ is executed after finishing $i), \Rightarrow s_{j} \geq s_{i}+d_{i}+t_{e_{i} b_{j}}$.

$$
s_{j} \geq s_{i}+d_{i}+t_{e_{i} b_{j}}-\left(3-y_{i c}-y_{j c}-n_{i j}\right) M_{T} \quad \forall i, j \in \mathcal{R}, i \neq j, \forall c \in \mathcal{C}
$$

The starting time of two requests handled by different cranes depends on whether they are 
conflicting or not. When the cranes' trajectories while handling two requests are not conflicting, then the requests' starting times do not influence each other. However, if the two requests are conflicting, a waiting time must be applied between starting the requests. $g_{i c j c^{\prime}}$ denotes the required waiting time between request $i$ handled by crane $c$ and request $j$ handled by crane $c^{\prime}$. The value of $g_{i c j c^{\prime}}$ depends upon the position of the cranes involved and on their movement direction resulting in three different cases reflected by Equations (19), (20) and (21).

The first case (Figure $5(\mathrm{a})$ occurs when $y_{i c}=y_{j c^{\prime}}=1$, crane $c$ is to the left of $c^{\prime}, \eta_{c}<\eta_{c^{\prime}}$ and $i$ 's destination is to the right of $j$ 's origin, $h_{e_{i}}>h_{b_{j}}$. In such situation crane $c^{\prime}$ cannot immediately begin executing $j$ after the starting time of $i$, and must instead wait for $c$ to finish $i, d_{i}$, plus the time $c$ requires to travel from $i$ 's destination to $j$ 's origin, $t_{e_{i} b_{j}}$. Therefore the waiting time of crane $c^{\prime}$ to execute request $j$ is $d_{i}+t_{e_{i} b_{j}}$. Another situation which results in the same value for $g_{i c j c^{\prime}}$ occurs when crane $c$ is to the right of $c^{\prime}, \eta_{c}>\eta_{c^{\prime}}$ and $i$ 's destination is to the left of $j$ 's origin, $h_{e_{i}}<h_{b_{j}}$ (Figure 5(b)]). Figure 5 presents these situations both resulting in $g_{i c j c^{\prime}}=d_{i}+t_{e_{i} b_{j}}$.

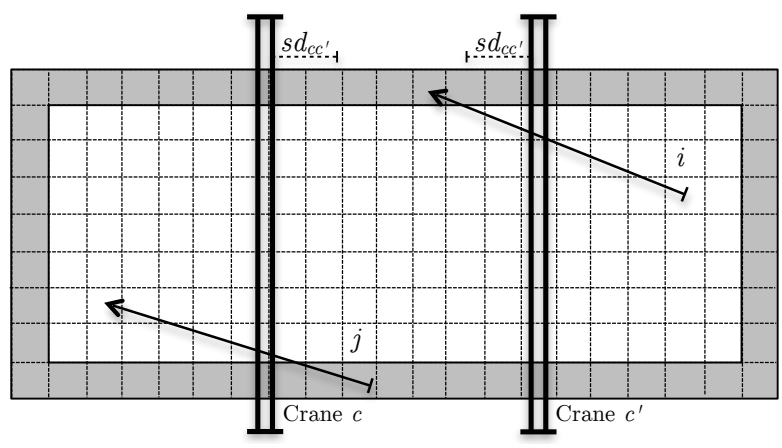

(a) $h_{e_{i}}>h_{b_{j}}, \eta_{c}<\eta_{c^{\prime}}$

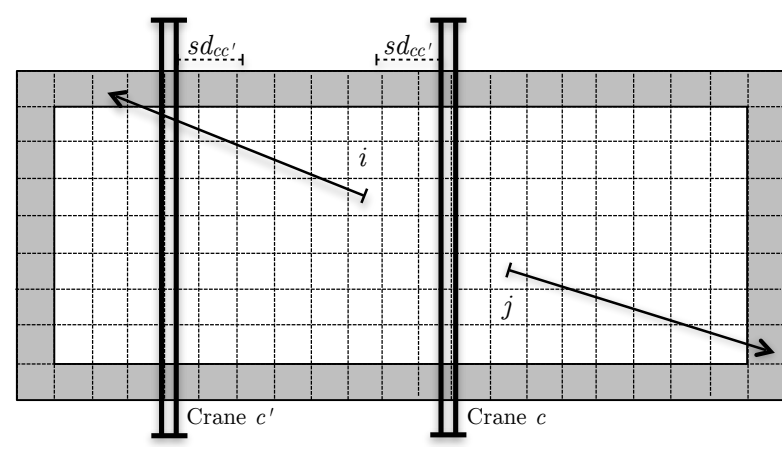

(b) $h_{e_{i}}<h_{b_{j}}, \eta_{c}>\eta_{c^{\prime}}$

Figure 5: Two conditions which result in $g_{i c j c^{\prime}}=d_{i}+t_{e_{i} b_{j}}$.

The value of $g_{i c j c^{\prime}}$ is determined by Equations (19).

$$
g_{i c j c^{\prime}}=d_{i}+t_{e_{i} b_{j}} \quad \forall i \in \mathcal{R},\left\{\begin{array}{l}
j \in \mathcal{R}: h_{e_{i}}>h_{b_{j}}, \forall c, c^{\prime} \in \mathcal{C}: \eta_{c}<\eta_{c^{\prime}} \\
j \in \mathcal{R}: h_{e_{i}}<h_{b_{j}}, \forall c, c^{\prime} \in \mathcal{C}: \eta_{c}>\eta_{c^{\prime}}
\end{array}\right.
$$

Conflicts may also occur when crane $c^{\prime}$ does not necessarily need to wait until the end of request $i$. Figure 6 illustrates examples where crane $c^{\prime}$ may begin executing request $j, t_{e_{i} b_{j}}$ time units before concluding request $i$. Such value for $g_{i c j c^{\prime}}$ guarantees that by the time crane $c$ finishes executing request $i$, crane $c^{\prime}$ 's distance to crane $c$ exceeds $s d_{c c^{\prime}}$. 


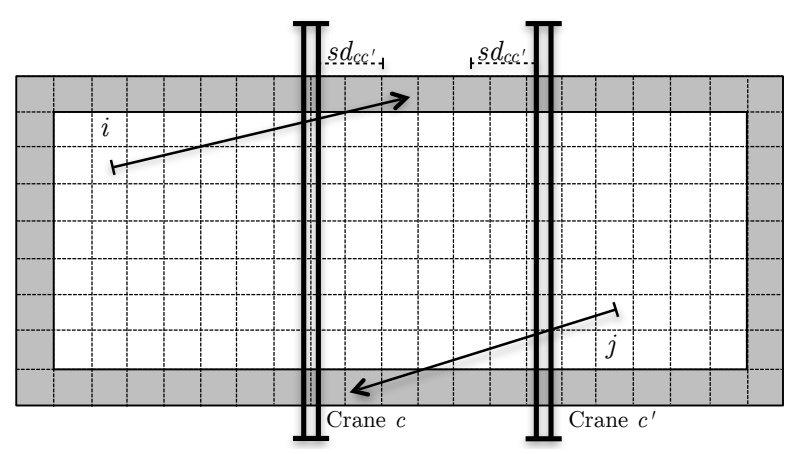

(a) $h_{b_{i}}<h_{e_{i}}<h_{b_{j}}, \eta_{c}<\eta_{c^{\prime}}$

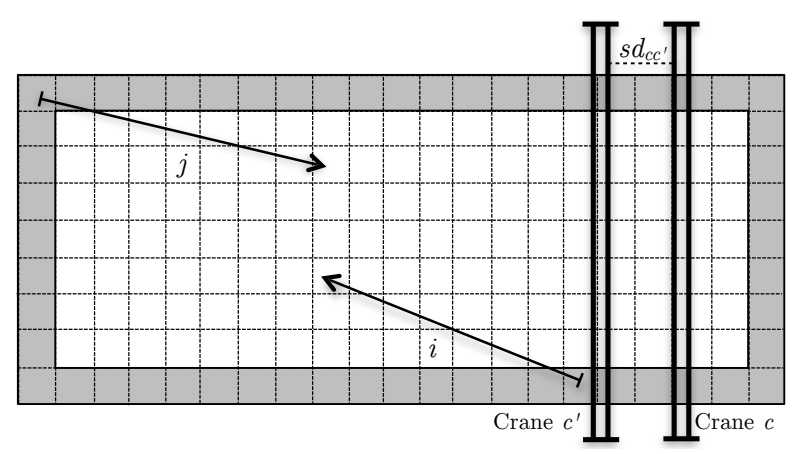

(b) $h_{b_{j}}<h_{e_{i}}<h_{b_{i}}, \eta_{c}>\eta_{c^{\prime}}$

Figure 6: Two conditions which result in $g_{i c j c^{\prime}}=d_{i}-t_{e_{i} b_{j}}$.

Equations 20 define the conditions for $g_{i c j c^{\prime}}=d_{i}-t_{e_{i} b_{j}}$.

$$
g_{i c j c^{\prime}}=d_{i}-t_{e_{i} b_{j}} \quad \forall i \in \mathcal{R},\left\{\begin{array}{l}
j \in \mathcal{R}: h_{b_{i}}<h_{e_{i}}<h_{b_{j}}, \forall c, c^{\prime} \in \mathcal{C}: \eta_{c}<\eta_{c^{\prime}} \\
j \in \mathcal{R}: h_{b_{j}}<h_{e_{i}}<h_{b_{i}}, \forall c, c^{\prime} \in \mathcal{C}: \eta_{c}>\eta_{c^{\prime}}
\end{array}\right.
$$

Figure 7 presents two other scenarios which influence the value of $g_{i c j c}$. The first scenario occurs when crane $c$ is to the left and $c^{\prime}$ is to the right, $\eta_{c}<\eta_{c^{\prime}}$, and the destination of request $i$ is to the left of $j$ 's origin, $h_{e_{i}}<h_{b_{j}}$ and $h_{e_{i}}<h_{b_{i}}$, or when crane $c^{\prime}$ is to the left of $c, \eta_{c}>\eta_{c^{\prime}}$, and the destination of $i$ is to the right of $j$ 's origin, $h_{e_{i}}>h_{b_{j}}$ and $h_{e_{i}}>h_{b_{i}}$. In these cases $g_{i c j c^{\prime}}$ is equal to the travel time from $i$ 's to $j$ 's origin, $t_{b_{i} b_{j}}$.

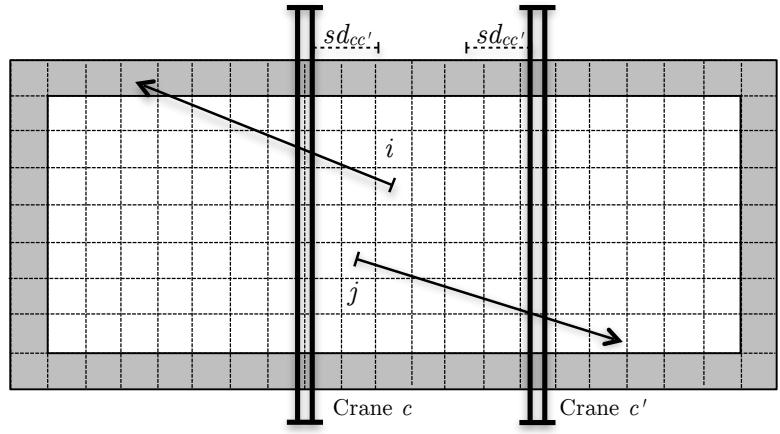

(a) $h_{e_{i}}<h_{b_{j}}, h_{e_{i}}<h_{b_{i}}, \eta_{c}<\eta_{c^{\prime}}$

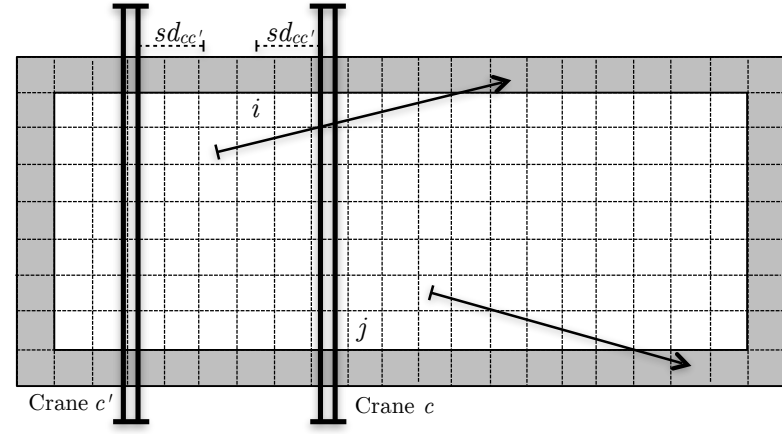

(b) $h_{e_{i}}>h_{b_{j}}, h_{e_{i}}>h_{b_{i}}, \eta_{c}>\eta_{c^{\prime}}$

Figure 7: Two conditions which result in $g_{i c j c^{\prime}}=t_{b_{i} b_{j}}$.

When $i$ is an output request and the conditions presented in Figure 7 are satisfied, Equations (21) determine the value of $g_{i c j c^{\prime}}$.

$$
g_{i c j c^{\prime}}=t_{b_{i} b_{j}} \quad \forall i \in \mathcal{R},\left\{\begin{array}{l}
j \in \mathcal{R}: h_{e_{i}}<h_{b_{j}}, h_{e_{i}}<h_{b_{i}}, \forall c, c^{\prime} \in \mathcal{C}: \eta_{c}<\eta_{c^{\prime}} \\
j \in \mathcal{R}: h_{e_{i}}>h_{b_{j}}, h_{e_{i}}>h_{b_{i}}, \forall c, c^{\prime} \in \mathcal{C}: \eta_{c}>\eta_{c^{\prime}}
\end{array}\right.
$$

Constraints (22) and (23) define the value of variable $q_{i j}$ which must equal 1 if request $i$ begins before $j$ 's starting time and 0 otherwise. 


$$
\begin{array}{ll}
s_{i} \geq s_{j}-M_{T} q_{i j} & \forall i, j \in \mathcal{R}: i \neq j \\
s_{i} \leq s_{j}+\left(1-q_{i j}\right) M_{T} & \forall i, j \in \mathcal{R}: i \neq j
\end{array}
$$

Assume requests $i$ and $j$ are handled by two separate cranes, $c$ and $c^{\prime}$. If the requests are not conflicting, $i$ 's starting time does not influence $j$ 's, since the requests are assigned to different cranes. If, however, the requests are conflicting, then the starting time of $j$ must consider $i$ 's starting time coupled with the position and direction of both cranes. Constraints (24) coordinate the starting times of consecutive requests if the following conditions are satisfied: $(i)$ two different cranes $\left(c\right.$ and $\left.c^{\prime}\right)$ are assigned to execute requests $i$ and $j, y_{i c}=y_{j c^{\prime}}=1,(i i)$ the requests are conflicting, $o_{i j}=1$, and (iii) request $j$ begins after request $i, q_{i j}=1$.

$$
s_{j} \geq s_{i}+g_{i c j c^{\prime}}+s t_{c c^{\prime}}-\left(4-y_{i c}-y_{j c^{\prime}}-o_{i j}-q_{i j}\right) M_{T} \quad \forall i, j \in \mathcal{R}, c, c^{\prime} \in \mathcal{C}, i \neq j, c \neq c^{\prime}
$$

When the three conditions are satisfied, then request $j$ must begin after the starting time of $i$ plus the waiting time required between requests $i$ and $j\left(g_{i c j c^{\prime}}\right)$ and the time required for a crane to travel the safety distance between $c$ and $c^{\prime}\left(s t_{c c^{\prime}}\right)$.

Constraints 25) ensure the starting time of request $i$ occurs after its release time $r_{i}$.

$$
s_{i} \geq r_{i} \quad \forall i \in \mathcal{R}
$$

When products are stacked on top of each other and the bottom one should be moved, the requests associated with the top products must precede those associated with products situated on the lower levels. A set of precedence constraints, $\Gamma_{i}$, indicates the set of requests which must precede $i$. Constraints (26) specify these precedence relations and state that request $i$ may only begin after all its preceding requests are finished.

$$
s_{i} \geq s_{j}+d_{j} \quad \forall i \in \mathcal{R}, j \in \Gamma_{i}
$$

The tardiness associated with request $i$ is denoted by $\delta_{i} \geq 0$. Constraints (27) set the delay of each request to be at least the request's finishing time $\left(s_{i}+d_{i}\right)$ minus its due time, $\tau_{i}$.

$$
\delta_{i} \geq s_{i}+d_{i}-\tau_{i} \quad \forall i \in \mathcal{R}
$$

All variables, except $s_{i}$ (starting time of request $i$ ) and $\delta_{i}$ (tardiness of request $i$ ), are binary:

$$
\begin{array}{ll}
y_{i c} \in\{0,1\} & \forall i \in \mathcal{R}, c \in \mathcal{C} \\
n_{i j}, q_{i j}, o_{i j}, o_{i j}^{a}, o_{i j}^{b}, o_{i j}^{c} \in\{0,1\} & \forall i, j \in \mathcal{R}: i \neq j \\
s_{i}, \delta_{i} \geq 0 & \forall i \in \mathcal{R}
\end{array}
$$

The CSP formulation is given by $\mathcal{F}_{C S}$, where the objective function (31) minimises the tardiness of all requests. 


$$
\mathcal{F}_{C S}\left\{\begin{array}{lc}
\text { min. } & \sum_{i \in \mathcal{R}} \delta_{i} \\
\text { s.t. } & (9)-30
\end{array}\right.
$$

\subsection{Integrated formulation for crane-operated warehouse scheduling problems}

This section introduces an integrated continuous-time formulation for the CWSP $\left(\mathcal{F}_{C W S}\right)$ which considers the location assignment for input requests, the crane assignment for all requests, and the sequencing of the requests per crane. $\mathcal{F}_{C W S}$ includes all constraints associated with the LAP and CSP plus some additional constraints. Since the input requests' destinations are undefined (whereas for the CSP, they are determined by first solving the LAP), the following parameters in $\mathcal{F}_{C S}$ become variables in $\mathcal{F}_{C W S}: h_{i}^{-}, h_{i}^{+}, d_{i}$ and $e_{i}$ for all requests $i \in \mathcal{R}^{I}$ and $o_{i j}^{a}$ when at least one of requests $i$ and $j$ is an input request. Note that since these variables are defined only for input requests, the definitions in Table 2 remain valid for output requests.

Additional constraints are required to assist in identifying the conflicting requests. Equations (32) and (33) are employed to obtain $h_{i}^{-}$and $h_{i}^{+}$for request $i \in \mathcal{R}^{I}$, respectively. Constraints (34) are employed to define the values of $o_{i j}^{a}$.

$$
\begin{array}{rlrl}
h_{i}^{-} & =\sum_{l \in L: h_{l} \leq h_{b_{i}}} h_{l} x_{i l}+\sum_{l \in L: h_{l}>h_{b_{i}}} h_{b_{i}} x_{i l} & & \forall i \in \mathcal{R}^{I} \\
h_{i}^{+} & =\sum_{l \in L: h_{l} \leq h_{b_{i}}} h_{b_{i}} x_{i l}+\sum_{l \in L: h_{l}>h_{b_{i}}} h_{l} x_{i l} & & \forall i \in \mathcal{R}^{I} \\
h_{i}^{+} \geq h_{j}^{-}-M_{L} \cdot o_{i j}^{a} & \forall i, j \in \mathcal{R}: i \neq j
\end{array}
$$

Output requests have a fixed duration, while input requests' durations depend on the chosen destination. Constraints (35) are employed to compute the duration of input requests.

$$
d_{i}=\sum_{l \in L} t_{b_{i} l} x_{i l} \quad \forall i \in \mathcal{R}^{I}
$$

As the destinations of input requests are decision variables, setting the value of $g_{i c j c^{\prime}}$ when request $i$ is an input request requires additional constraints. Equations (36) define the value of $g_{i c j c^{\prime}}$ when crane $c$ handling request $i$ is to the left of crane $c^{\prime}$ which handles request $j$. When $h_{e_{i}}<h_{b_{j}}$ and $h_{e_{i}}<h_{b_{i}}$ then $g_{i c j c^{\prime}}=t_{b_{i} b_{j}}$ as in Constraints (21), if $h_{e_{i}}<h_{b_{j}}$ and $h_{e_{i}}>h_{b_{i}}$ then $g_{i c j c^{\prime}}=d_{i}-t_{e_{i} b_{j}}$ as in Constraints (20), and finally $h_{e_{i}}>h_{b_{j}}$ then $g_{i c j c^{\prime}}=d_{i}+t_{e_{i} b_{j}}$ as in Constraints (19).

$$
\begin{gathered}
g_{i c j c^{\prime}}=\sum_{l \in L: h_{l}<h_{b_{j}}, h_{l}<h_{b_{i}}} t_{b_{i} b_{j}} x_{i l}+\sum_{l \in L: h_{l}<h_{b_{j}}, h_{l}>h_{b_{i}}}\left(d_{i}-t_{e_{i} b_{j}}\right) x_{i l}+\sum_{l \in L: h_{l}>h_{b_{j}}}\left(d_{i}+t_{e_{i} b_{j}}\right) x_{i l} \\
\forall i \in \mathcal{R}^{I}, j \in \mathcal{R}, \forall c, c^{\prime} \in \mathcal{C}: \eta_{c}<\eta_{c^{\prime}}
\end{gathered}
$$

Similarly, Equations (37) set the value of $g_{i c j c^{\prime}}$ for input requests in case crane $c$ handling request $i$ is to the right of crane $c^{\prime}$ which handles request $j$. 


$$
\begin{gathered}
g_{i c j c^{\prime}}=\sum_{l \in L: h_{l}<h_{b_{j}}}\left(d_{i}+t_{e_{i} b_{j}}\right) x_{i l}+\sum_{l \in L: h_{l}>h_{b_{j}}, h_{l}<h_{b_{i}}}\left(d_{i}-t_{e_{i} b_{j}}\right) x_{i l}+\sum_{l \in L: h_{l}>h_{b_{j}}, h_{l}>h_{b_{i}}} t_{b_{i} b_{j}} x_{i l} \\
\forall i \in \mathcal{R}^{I}, j \in \mathcal{R}, \forall c, c^{\prime} \in \mathcal{C}: \eta_{c}>\eta_{c^{\prime}}
\end{gathered}
$$

In case of output requests, as their destinations are given, the value of $g_{i c j c^{\prime}}$ is obtained in a similar way as in $\mathcal{F}_{\mathcal{C S}}$. Constraints (38), (39) and (40) are modified based on Constraints (19), (20) and (21) respectively to set $g_{i c j c^{\prime}}$ for request $i \in \mathcal{R}^{O}$ and request $j \in \mathcal{R}$.

$$
\begin{array}{ll}
g_{i c j c^{\prime}}=t_{b_{i} b_{j}} & \forall i \in \mathcal{R}^{O},\left\{\begin{array}{l}
j \in \mathcal{R}: h_{e_{i}}<h_{b_{i}}, h_{e_{i}}<h_{b_{j}}, \forall c, c^{\prime} \in \mathcal{C}: \eta_{c}<\eta_{c^{\prime}} \\
j \in \mathcal{R}: h_{b_{i}}<h_{e_{i}}, h_{b_{j}}<h_{e_{i}}, \forall c, c^{\prime} \in \mathcal{C}: \eta_{c}>\eta_{c^{\prime}}
\end{array}\right. \\
g_{i c j c^{\prime}}=d_{i}-t_{e_{i} b_{j}} & \forall i \in \mathcal{R}^{O},\left\{\begin{array}{l}
j \in \mathcal{R}: h_{b_{i}}<h_{e_{i}}<h_{b_{j}}, \forall c, c^{\prime} \in \mathcal{C}: \eta_{c}<\eta_{c^{\prime}} \\
j \in \mathcal{R}: h_{b_{i}}>h_{e_{i}}>h_{b_{j}}, \forall c, c^{\prime} \in \mathcal{C}: \eta_{c}>\eta_{c^{\prime}}
\end{array}\right. \\
g_{i c j c^{\prime}}=d_{i}+t_{e_{i} b_{j}} & \forall i \in \mathcal{R}^{O},\left\{\begin{array}{l}
j \in \mathcal{R}: h_{e_{i}}>h_{b_{j}}, \forall c, c^{\prime} \in \mathcal{C}: \eta_{c}<\eta_{c^{\prime}} \\
j \in \mathcal{R}: h_{e_{i}}<h_{b_{j}}, \forall c, c^{\prime} \in \mathcal{C}: \eta_{c}>\eta_{c^{\prime}}
\end{array}\right.
\end{array}
$$

Another set of additional constraints is required due to implied precedence constraints during the location assignment. Constraints (41) assert that if an input request is assigned atop another input request, the bottom request is placed first. Constraints (42) ensure $j$ is moved after $i$ if it has been assigned to $i$ 's origin location.

$$
\begin{array}{ll}
n_{i j} \geq x_{j l}+x_{i k}-1 & \forall i, j \in \mathcal{R}^{I}: i \neq j, l \in L, k \in U_{l} \\
n_{i j} \geq x_{j b_{i}} & \forall i \in \mathcal{R}, j \in \mathcal{R}^{I}
\end{array}
$$

The objective function is a weighted linear combination of the LAP's objectives (Equation (2)) and those of the CSP (Equation (31)). The CWSP formulation is given by $\mathcal{F}_{C W S}$ :

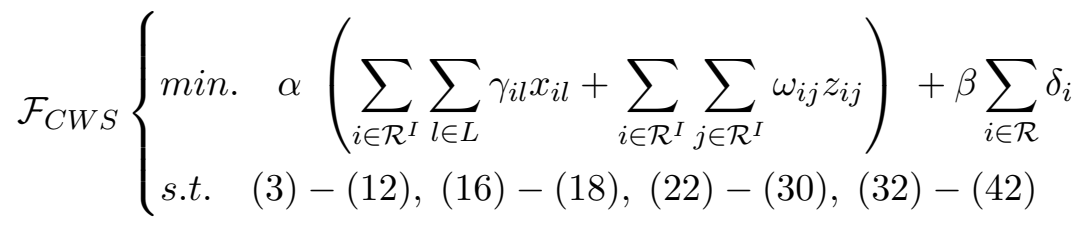

The CWSP is a complex problem that generalizes the Sequential Ordering Problem (SOP) (Escudero, 1988), which represents a special case of the CWSP scheduling component with a single crane and fixed request destinations. Consequently, the CWSP is considered at least as hard as the SOP. Given the SOP is known to be $\mathcal{N} \mathcal{P}$-hard (Montemanni et al., 2009), by consequence, CWSP is an $\mathcal{N} \mathcal{P}$-hard problem.

\section{Heuristic approach}

A local search based algorithm is proposed consisting of constructive and improvement phases for solving the CWSP. During both phases, an indirect solution representation capable of reducing 
the search space is employed (Section 4.1). All the algorithm's components are explained throughout the following sections. The constructive phase $(4.2)$ consists of a greedy constructive heuristic inspired by a set of dispatching rules. During the improvement phase (4.3) a Late Acceptance Hill Climbing (LAHC) meta-heuristic (Burke and Bykov, 2017) is employed which considers several neighbourhood structures (4.4). Figure 8 presents a general overview of the proposed algorithm.

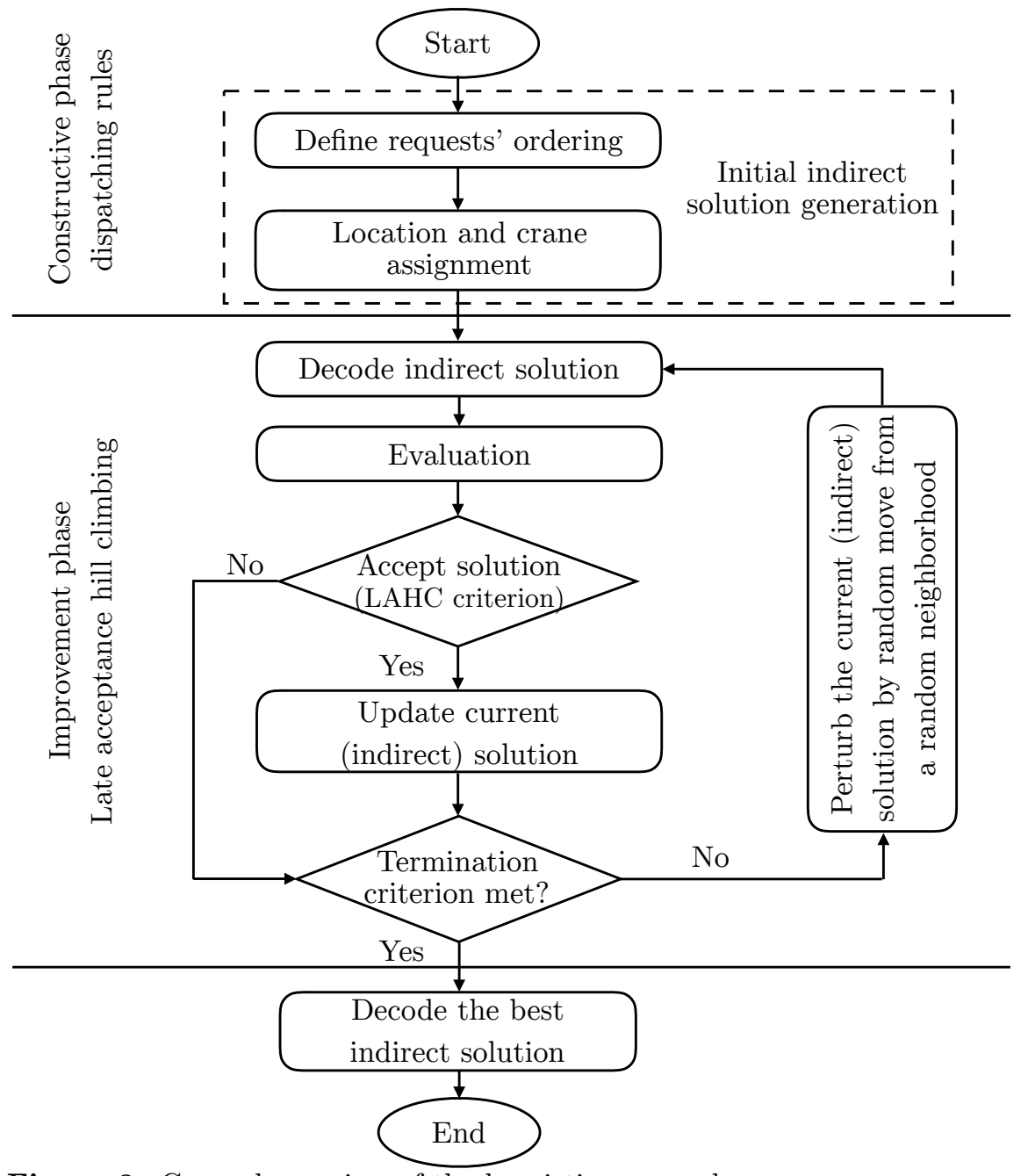

Figure 8: General overview of the heuristic approach

\subsection{Solution representation}

The local search-based algorithm considers an indirect solution representation. Each solution is represented by a list $\mathcal{L}$ of (request, location, crane)-tuples. The actual schedule is produced by a decoder which utilizes both the ordering of requests, the locations and crane assignments included in list $\mathcal{L}$.

The decoder is also employed to evaluate solutions by computing their storage cost and total tardiness. Whereas the total tardiness can be obtained by applying Equation (2), computing the total tardiness is less straightforward. It requires all requests' starting times, which determine on the requests' execution order in $\mathcal{L}$ in such a way that all conflict-related constraints are satisfied. 
The decoder is presented by Algorithm 1 . For each tuple $\left(i, e_{i}, c\right)$ in $\mathcal{L}$, request $i$ has its starting time $s_{i}$ initialized as its release time (lines 1-2). If crane $c$ was previously assigned to another request, the starting time $s_{i}$ is set to be after the execution of the crane's previous request plus the time required for the crane to begin executing request $i$ (lines 3-5). To handle conflicts with requests assigned to other cranes, the algorithm loops over the schedule of all other cranes from end to beginning (lines 6-8). In case request $i$ conflicts with a request $k$ of crane $c^{\prime}$ 's schedule, $s_{i}$ is set to be at least $s_{k}+g_{k c^{\prime} i c}+s t_{c c^{\prime}}$ (lines 9-10), where $g_{k c^{\prime} i c}$ is the waiting time defined in Section 3 . Once the algorithm finds a conflicting request $k$ in crane $c^{\prime \prime}$ s schedule, it is unnecessary to proceed checking, as all other requests assigned to $c^{\prime}$ are scheduled earlier than $k$. The algorithm then moves on to the next crane, if any remain. Therefore, the decoder will execute only $O(|\mathcal{R}| \times|\mathcal{C}|)$ operations in the best case. Since in most applications the number of cranes $|C|$ may be fixed as a constant, in the best case the decoder runs in linear time. In the worst case, however, $O\left(|\mathcal{R}|^{2}\right)$ operations may be required by the decoder. After the schedules of all cranes are checked, the minimum value for $s_{i}$ is calculated and the algorithm includes request $i$ into crane $c$ 's schedule (lines 12). Once all starting times are computed, the tardiness can be easily calculated employing Equations (27) and (31).

One of the advantages of the indirect solution representation proposed is that it prevents the generation of a class of unattractive solutions which include avoidable idle times between operations. Indeed, given that the indirect representation is no more than a sequence, the decoder will produce a left-active schedule which is always better than alternative schedules based on this sequence, but which include idle times.

Moreover, the indirect solution is simple to modify, requiring only a few verifications to guarantee feasibility. Its main disadvantage lies in the additional $O\left(|R|^{2}\right)$ operations required for decoding and evaluating solutions.

\subsection{Constructive heuristic}

An initial solution is constructed by a greedy algorithm which applies a set of dispatching rules. Algorithm 2 details this procedure. A directed acyclic graph is considered where nodes represent

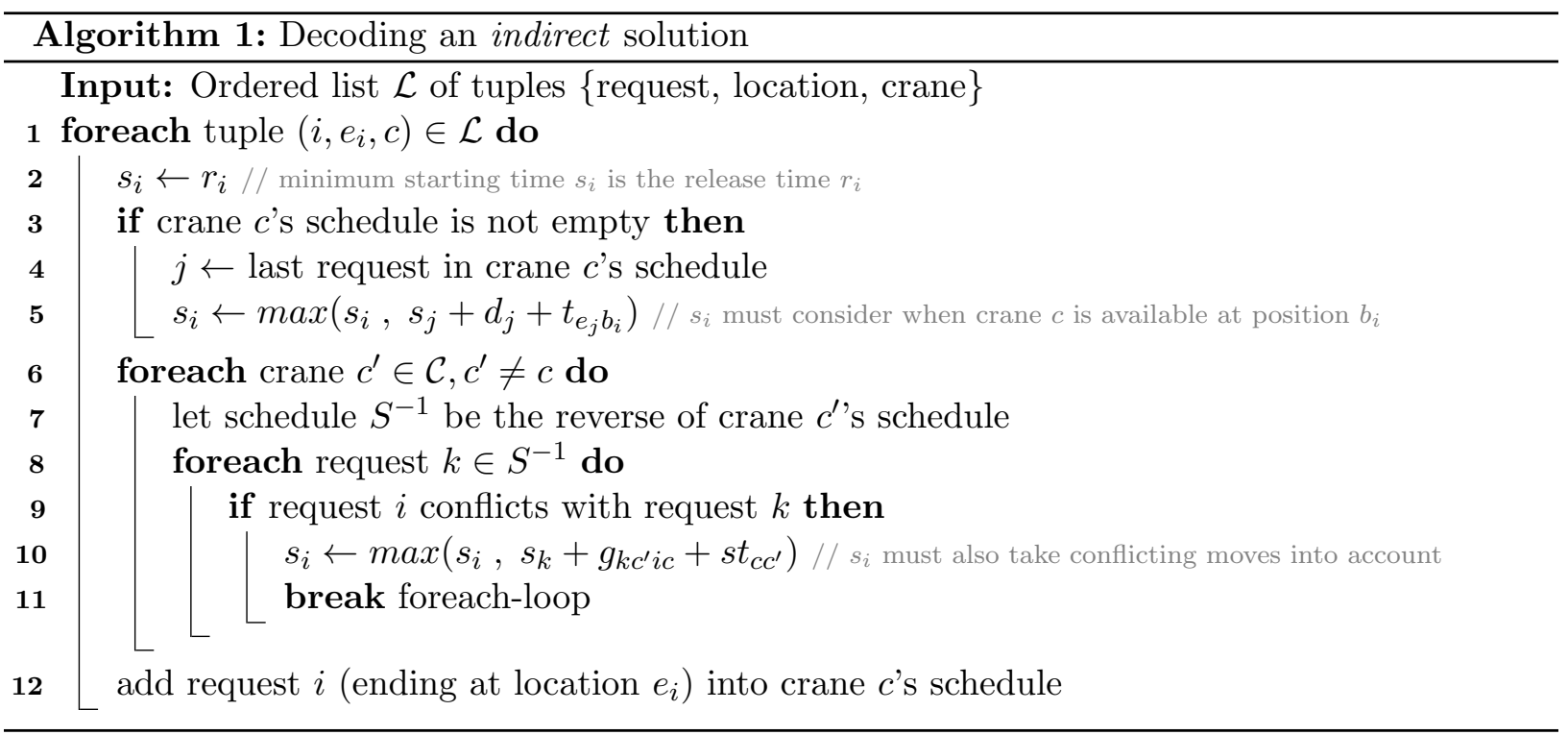


requests and arcs directing from $r_{i}$ to $r_{j}$ indicate precedence constraints $\left(r_{i}\right.$ must precede $\left.r_{j}\right)$. In this graph, requests are first sorted topologically. By sorting the directed acyclic graph topologically, for every directed $\operatorname{arc}\left(r_{i}, r_{j}\right), r_{i}$ precedes $r_{j}$ in the ordering ensuring that the precedence constraints are respected. Requests within the same topological level are sorted by their release times. This strategy defines the initial ordering of the requests (line 1). The solution is initialized as an empty list (line 2) and, afterwards, two steps are applied for each request (lines 3-6). First, the available location which has the lowest storage cost is assigned to input requests (lines 4-5). Note that output requests have preassigned locations and therefore do not require such an assignment. In the second step, requests are assigned to the nearest available crane (line 6). Both the requests' starting times and the cost of the solution corresponding with $\mathcal{L}$ are computed employing the decoder presented in Algorithm 1.

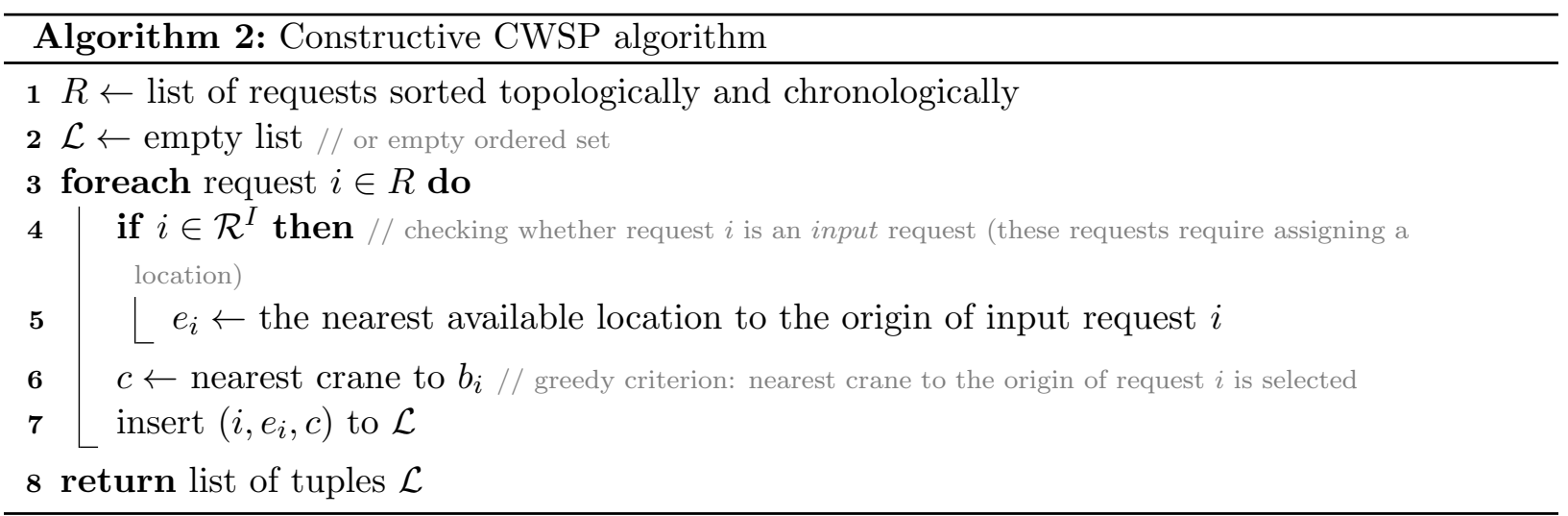

\subsection{Late Acceptance Hill Climbing}

The LAHC meta-heuristic represents an extension of the greedy hill-climbing algorithm which compares the candidate solution against the solution which was 'current' $l$ iterations before. Consequently, the meta-heuristic permits the acceptance of worsening solutions, thus avoiding local optima. This study employs the LAHC meta-heuristic presented in Algorithm 3 which requires the following arguments: (i) initial solution $s_{0},(i i)$ parameter $l,($ iii $)$ set of neighbourhoods $\mathcal{N}$, $(i v)$ maximum number of iterations without improvement $i t^{\max }$ and $(v)$ timeout, which indicates the runtime of LAHC.

The LAHC meta-heuristic maintains a fixed-length list $v$ containing objective function values of the solutions visited during the last $l$ iterations. Initially, all $v$ elements are set to the initial solution's objective value, given by $f\left(s_{0}\right)$ (line 1). Next, current solution $s$, best solution $s^{*}$ and index $i$ are initialized (lines 2-3). At each iteration a new candidate solution $s^{\prime}$ is generated by applying a randomly generated move from a randomly selected neighbourhood to the current solution $s$ (lines 5-6). The candidate solution's objective value, $f\left(s^{\prime}\right)$, is compared against $v_{i}$ and the current solution's value $f(s)$ (line 7 ). $s^{\prime}$ is accepted (line 12) to replace the current solution if its objective value is less than or equal to $v_{i}$ or $f(s)$. If the candidate solution $s^{\prime}$ has a better objective value than the best solution $s^{*}$ generated thus far, it replaces $s^{*}$ (lines 13-14). If the objective value of $s^{\prime}$ is greater than $v_{i}$ or $f(s)$, the number of iterations without improvement, it, increments by one (lines 15-16). Finally, $v_{i}$ and $i$ are updated: $v_{i} \leftarrow f(s)$ (replacing the oldest value), and $i$ is set to point to the next position of list $l$ (lines 17-18). Note that $i$ acts as a cyclic 


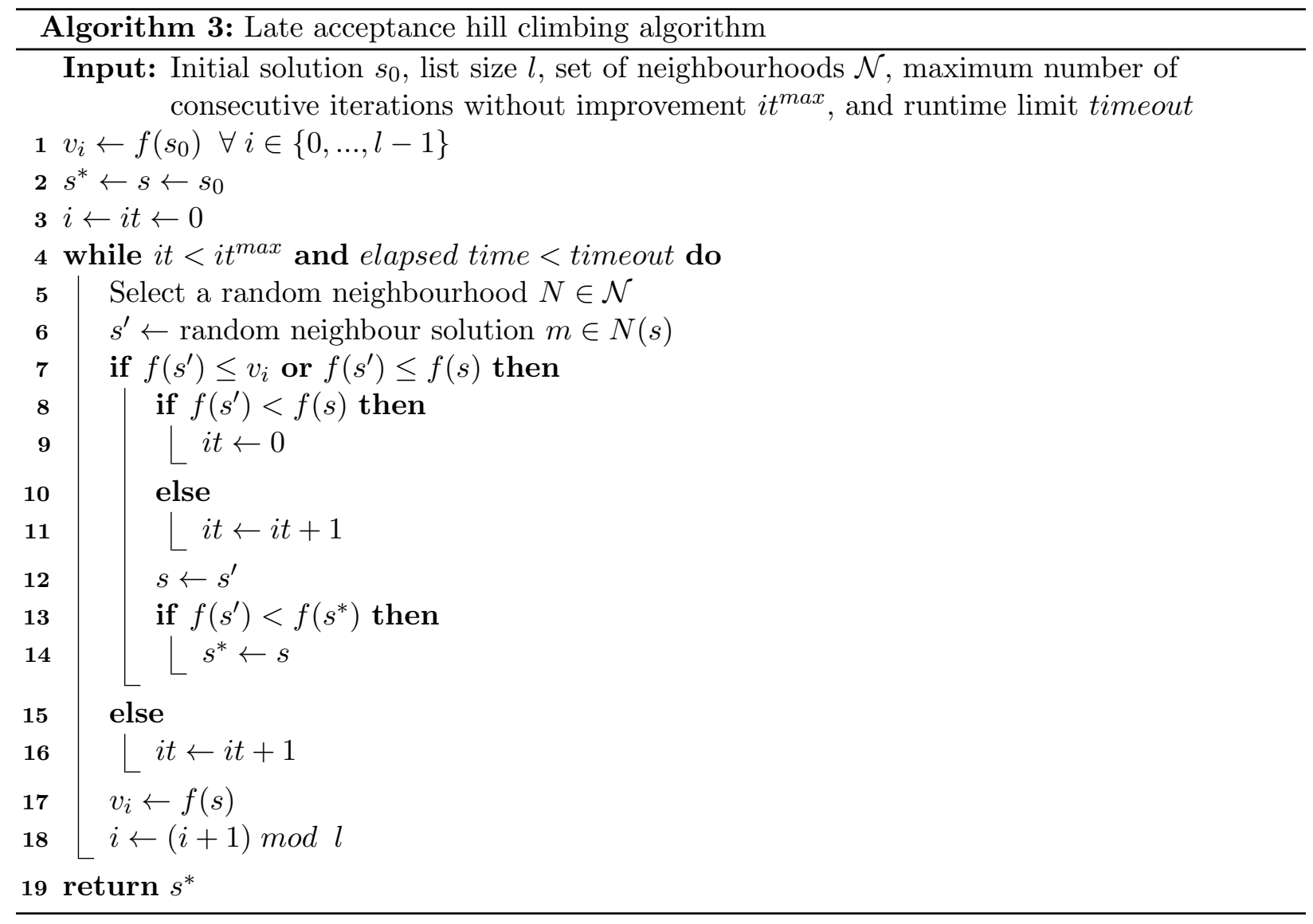

pointer. This iterative process repeats until the elapsed time reaches timeout or the number of iterations without improvement reaches $i t^{\max }$. The latter criterion prevents the heuristic from continuing the search in situations whre no more improvements are generated during a long period of time. Finally, the best solution obtained is then returned (line 19).

\section{$4.4 \quad$ Neighbourhood structures}

Ten neighbourhoods were developed to explore the CWSP's solution space. The neighbourhoods are grouped into two categories: $(i)$ Location assignment and (ii) Crane scheduling neighbourhoods. All ten neighbourhoods operate over the list of tuples $\mathcal{L}$ and therefore modify only the indirect solution. Infeasible solutions may be obtained by applying some of these neighbourhood operators to a solution. For instance, both assigning a product atop an empty location and ignoring the precedence constraints when changing the requests' order result in infeasibility. Such infeasible solutions are discarded during the search.

The ten neighbourhoods are detailed as follows.

\section{Location assignment neighbourhoods}

- Location Re-assignment (LR): a random input request is selected and its destination location is replaced with another randomly selected available location. 
- Location Swap (LS): two random input requests are selected and their destination locations are swapped.

- Greedy Location Assignment (GLA): a set of input requests is randomly selected and their location assignments are removed. Then, all possible locations are considered and the requests are greedily assigned to the lowest-cost location.

\section{Crane scheduling neighbourhoods}

- Crane Re-assignment (CR): a set of three requests is selected randomly and all crane assignment combinations are enumerated. The resulting solution is the one with the best quality among the enumerated solutions.

- Order Swap (OS): two requests are randomly selected and their tuples are swapped, changing their execution order.

- Random Insertion (RI): a random tuple is removed and re-inserted into a random position in the list.

- Random Best Insertion (RBI): a request is randomly selected and its tuple is inserted into the lowest-cost position within the assigned crane's schedule. Note that this neighbourhood requires $O(|R|)$ operations to identify the lowest-cost position.

- Nearest Location Assignment (NLA): a set of input requests is randomly selected and their location assignments are removed. Then, all possible locations are considered and each request is greedily assigned to the location nearest to its origin.

This neighbourhood is employed as a crane scheduling neighborhood since it reduces the duration of requests and, as a consequence, reduces the potential risk of generating conflicting requests.

- Best Order Permutation (BOP): a range of requests in a crane's schedule is randomly selected; their tuples are subsequently removed and the best permutation of the selected tuples, determined by enumeration, is inserted into the list.

This neighbourhood has exponential complexity and therefore the range must be limited to prevent prohibitive runtimes.

- Moving Best Order Permutation (MBOP): This neighbourhood begins from the first tuple in the list $\mathcal{L}$ and executes the BOP move within a range of three tuples. The procedure moves forward in $\mathcal{L}$ by one tuple and executes the BOP move for next three tuples. It ends after $|\mathcal{L}|-2$ iterations by executing the BOP move for the last three tuples in the list.

\section{Computational study}

This section investigates the impact of integrating the location assignment and crane scheduling problems. The performance of both the formulations and heuristic are assessed across different scenarios: sequential and integrated.

The sequential approach, on the one hand, solves two problems. It begins by solving the LAP, after which the assignments obtained are fixed when solving the CSP. The integrated approach, on the other hand, solves only one large problem: the CWSP. 
Table 3: Summary of sequential and integrated approaches

\begin{tabular}{lcll}
\hline & Formulation & Objective Function & LAHC Neighbourhoods \\
\hline \multirow{2}{*}{ Sequential approach } & $\mathcal{F}_{L A}$ & Equation (2) & LR, LS, GLA \\
& $\mathcal{F}_{C S}$ & Equation (31) & CR, OS, RI, RBI, BOP, MBOP \\
\multirow{2}{*}{ Integrated approach } & $\mathcal{F}_{C W S}$ & Equation (43) & $\begin{array}{l}\text { LR, LS, GLA, CR, OS, RI, } \\
\text { RBI, NLA, BOP, MBOP }\end{array}$ \\
\hline
\end{tabular}

Table 3 summarizes the formulations, objective functions and neighbourhood structures employed by each approach. The sequential approach employs formulations $\mathcal{F}_{L A}$ and $\mathcal{F}_{C S}$ to solve the LAP and CSP, respectively. It also utilizes Equation (2) and location assignment neighbourhoods to address the LAP heuristically. To solve the CSP by LAHC, the sequential approach employs Equation (31) and crane scheduling neighbourhoods. Whereas, the integrated approach solves formulation $\mathcal{F}_{C W S}$ and considers Equation (43) and all the neighbourhoods when employing LAHC. Table 3 summarizes the objective functions and neighbourhoods employed in the heuristic approaches to both the sequential and integrated problems.

The remainder of this section is organized as follows. First Section 5.1 presents the set of benchmark instances considered throughout the experiments. The comparison of the sequential and integrated approaches by employing the MIP formulations is presented in Section 5.2, while Section 5.3 presents the results of the sequential and integrated approaches employing the heuristic. Finally, Section 5.4 presents a discussion on the weights $\alpha$ and $\beta$ employed within the objective function (see Equation (1)) in addition to an analysis of the instances.

\subsection{Instances}

A set of benchmark instances was generated in correspondance with the data obtained from a real-world warehouse. These instances are available online ${ }^{1}$ to enable transparent comparison of the proposed formulations and algorithms. Four characteristics were considered for the instance generation:

Number of requests: the benchmark set includes instances with five levels denoting the number of requests: $10,20,30,50$ and 70 .

Storage size: the dataset considers three storage sizes. Small size storage areas have 250 $(10 \times 25)$ locations per level. Medium size storage areas have $525(15 \times 35)$ locations per level and large storage areas $1000(20 \times 50)$ locations per level. A large storage area may have up to 5000 locations. Based on the data obtained from the real-world problem, a realistic storage area contains approximately 3000 locations.

Maximum stacking level: this limit is imposed for each storage area depending on various criteria such as the product type and safety requirements. Three stacking levels are considered: one, three and five levels, where one denotes the ground level.

Storage load: this value is defined as a percentage indicating the initial occupancy level of the storage area. Three load factor values are considered: $30 \%, 50 \%$ and $70 \%$.

\footnotetext{
${ }^{1}$ CWSP data instances, solutions \& validator to be made publicly available in a data repository after paper acceptance due to incompatibility with double-blind review process. The data repository is available at a public url shared with the editor through the title-page.
} 

used in the instance generator.

Table 4: Instance generator distributions and parameters

\begin{tabular}{lll}
\multicolumn{2}{l}{ Table 4: Instance generator distributions and parameters } \\
\hline Instance Parameter & Distribution & Distribution Parameter \\
\hline Release time & Poisson & mean $=26.5$ \\
Time windows & Log normal & $\begin{array}{l}\text { mean }=0.0666^{*} \text { storage area's length } \\
\text { standard deviation }=0.1 * \text { storage area's width }\end{array}$ \\
Stored product location & Uniform & range $=$ locations in the storage area \\
Storage cost & Uniform & range $=[0,50)$ \\
\hline
\end{tabular}

The value of each attribute is reflected in the instance name, which indicates, from left to right: (i) number of requests, (ii) storage size, represented by the letters $s, m$ and $l$ signifying small, medium and large, respectively, (iii) maximum stacking level, and (iv) storage load. Instance name 50s_1_30, for example, indicates an instance with 50 requests, a small yard, maximum stacking level 1 , and storage load equal to $30 \%$.

Release times were generated according to a Poisson distribution with a fixed "average frequency" within the scheduling horizon. The scheduling horizon is computed by multiplying "Number of requests" by "average frequency". Due times were calculated as the sum of a request's "release time" and its "time window", wherein the "time window" was generated according to a log-normal distribution.

The number of yard products is calculated based on the yard size and yard load. For each stored product a location in the yard is randomly selected.

The storage cost of assigning an input request to a neighbouring location of any product is generated according to a uniform distribution. The cranes' travel times are measured by the Chebychev distance (the maximum of the lateral and longitudinal distance). It is assumed that the lateral and longitudinal speed of the cranes is identical, and thus the travel time may be substituted by the Chebychev distance. Table 4 summarizes the parameters of the distributions

The cranes are initially lined-up according to the required safety distance. The crane's travel speed is set to one time unit per horizontal coordinate.

\subsection{Comparison of sequential and integrated approaches by MIP formulations}

In the sequential approach, the LAP completely disregards the possible impact of attaining good solutions for the CSP. The integrated approach, however, enables a trade-off between the LAP and the CSP by setting different values for their corresponding weights $\alpha$ and $\beta$ in the objective function, Equation (1). Two settings are considered: $(i) \alpha>>\beta$, making the integrated approach put the highest priority to the LAP. This enables determining the benefit of an integrated approach over the sequential approach where both primarily focus on optimizing the storage cost. (ii) $\alpha=\beta$, which considers equal importance for storage cost and delays.

The mathematical formulations were solved by Gurobi Optimizer 7.5 and run on an Intel@ Xeon $\AA$ CPU E5-2650 v2 @ 2.6GHz with one hour runtime limit. Table 5 reports the results obtained by solving the sequential $\left(\mathcal{F}_{L A}+\mathcal{F}_{C S}\right)$ and integrated $\left(\mathcal{F}_{C W S}\right)$ formulations. The results of unsolved instances (no integer solution found within the runtime limit) are excluded from the table for brevity. Location assignment evaluation $\left(E_{L A}\right)$, crane scheduling evaluation $\left(E_{C S}\right)$ and total 
weighted $\operatorname{cost}\left(E_{\tau}\right)$, both when $\alpha=\beta$ and $\alpha>>\beta$, are compared. Emboldened numbers indicate that optimal solutions were obtained. When both the LAP and CSP are solved to optimality only $E_{\tau}$ is emboldened.

Table 5: Computational results obtained by solving the mathematical formulations (considered scenarios: $\alpha=100000, \beta=1(\alpha>>\beta)$ and $\alpha=\beta=0.50)$.

\begin{tabular}{|c|c|c|c|c|c|c|c|c|c|c|}
\hline \multirow[b]{2}{*}{ Instance } & \multicolumn{4}{|c|}{$\mathcal{F}_{L A}+\mathcal{F}_{C S}$} & \multicolumn{3}{|c|}{$\mathcal{F}_{C W S}(\alpha>>\beta)$} & \multicolumn{3}{|c|}{$\mathcal{F}_{C W S}(\alpha=\beta)$} \\
\hline & $E_{L A}$ & $E_{C S}$ & $E_{\tau}(\alpha>>\beta)$ & $E_{\tau}(\alpha=\beta)$ & $E_{L A}$ & $E_{C S}$ & $E_{\tau}$ & $E_{L A}$ & $E_{C S}$ & $E_{\tau}$ \\
\hline 10s_1_30 & 0 & 267.03 & $2.670 \mathrm{E}+02$ & 133.51 & 0 & 55.13 & $5.513 \mathrm{E}+01$ & 1 & 51.53 & 26.26 \\
\hline 10m_1_30 & 0 & 478.41 & $4.784 \mathrm{E}+02$ & 239.20 & 0 & 37.62 & $3.762 \mathrm{E}+01$ & 0 & 37.62 & 18.81 \\
\hline 101_1_30 & 0 & 515.69 & $5.157 \mathrm{E}+02$ & 257.84 & 0 & 76.82 & $7.682 \mathrm{E}+01$ & 0 & 76.82 & 38.41 \\
\hline 10s_1_50 & 0 & 229.25 & $2.293 \mathrm{E}+02$ & 114.63 & 0 & 123.93 & $1.239 \mathrm{E}+02$ & 11 & 78.92 & 44.96 \\
\hline 10m_1_50 & 0 & 219.18 & $2.192 \mathrm{E}+02$ & 109.59 & 0 & 56.45 & $5.645 \mathrm{E}+01$ & 7 & 40.07 & 23.53 \\
\hline 101_1_50 & 0 & 447.85 & $4.479 \mathrm{E}+02$ & 223.92 & 0 & 44.49 & $4.449 \mathrm{E}+01$ & 4 & 32.49 & 18.24 \\
\hline 10s_1_70 & 132 & 186.97 & $1.320 \mathrm{E}+07$ & 159.49 & 132 & 135.97 & $1.320 \mathrm{E}+07$ & 156 & 59.25 & 107.62 \\
\hline 10m_1_70 & 48 & 348.86 & $4.800 \mathrm{E}+06$ & 198.43 & 48 & 449.55 & $4.800 \mathrm{E}+06$ & 86 & 185.92 & 135.96 \\
\hline 101_1_70 & 11 & 271.44 & $1.100 \mathrm{E}+06$ & 141.22 & 11 & 148.17 & $1.100 \mathrm{E}+06$ & 25 & 73.78 & 49.39 \\
\hline 10s_3_30 & 13 & 352.10 & $1.300 \mathrm{E}+06$ & 182.55 & 13 & 179.72 & $1.300 \mathrm{E}+06$ & 49 & 76.07 & 62.53 \\
\hline 10m_3_30 & 1 & 354.66 & $1.004 \mathrm{E}+05$ & 177.83 & 1 & 107.51 & $1.001 \mathrm{E}+05$ & 9 & 70.37 & 39.68 \\
\hline 101_3_30 & 0 & 295.28 & $2.953 \mathrm{E}+02$ & 147.64 & 0 & 79.51 & $7.951 \mathrm{E}+01$ & - & - & - \\
\hline 10s_3_50 & 53 & 317.77 & $5.300 \mathrm{E}+06$ & 185.39 & 53 & 201.77 & $5.300 \mathrm{E}+06$ & 64 & 109.83 & 86.91 \\
\hline 10m_3_50 & 31 & 409.77 & $3.100 \mathrm{E}+06$ & 220.39 & 31 & 332.35 & $3.100 \mathrm{E}+06$ & 41 & 126.77 & 83.88 \\
\hline 101_3_50 & 9 & 454.43 & $9.005 \mathrm{E}+05$ & 231.72 & 9 & 145.71 & $9.001 \mathrm{E}+05$ & 12 & 85.91 & 48.95 \\
\hline 10s_3_70 & 130 & 392.07 & $1.300 \mathrm{E}+07$ & 187.73 & 130 & 279.13 & $1.300 \mathrm{E}+07$ & 190 & 102.40 & 146.20 \\
\hline 10m_3_70 & 64 & 433.66 & $6.400 \mathrm{E}+06$ & 248.83 & 64 & 492.57 & $6.400 \mathrm{E}+06$ & 93 & 77.90 & 85.45 \\
\hline 101_3_70 & 98 & 441.02 & $9.800 \mathrm{E}+06$ & 269.51 & 98 & 566.37 & $9.800 \mathrm{E}+06$ & 134 & 118.11 & 126.05 \\
\hline 10s_5_30 & 18 & 322.97 & $1.800 \mathrm{E}+06$ & 170.48 & 18 & 182.53 & $1.800 \mathrm{E}+06$ & 35 & 93.23 & 64.11 \\
\hline 10m_5_30 & 653 & 477.18 & $6.530 \mathrm{E}+07$ & 565.09 & 9 & 275.46 & $9.002 \mathrm{E}+05$ & 24 & 76.36 & 50.18 \\
\hline 101_5_30 & 464 & 457.64 & $4.640 \mathrm{E}+07$ & 460.82 & 6 & 244.62 & $6.002 \mathrm{E}+05$ & 16 & 206.88 & 111.44 \\
\hline 10s_5_50 & 66 & 435.57 & $6.600 \mathrm{E}+06$ & 250.79 & 66 & 292.55 & $6.600 \mathrm{E}+06$ & - & - & - \\
\hline 10m_5_50 & 25 & - & - & - & 25 & 294.46 & $2.500 \mathrm{E}+06$ & 42 & 196.59 & 119.30 \\
\hline 101_5_50 & 13 & 602.07 & $1.301 \mathrm{E}+06$ & 307.54 & 13 & 159.11 & $1.300 \mathrm{E}+06$ & 21 & 82.93 & 51.96 \\
\hline 10s_5_70 & 83 & 247.93 & $8.300 E+06$ & 165.64 & 83 & 247.93 & $8.300 \mathrm{E}+06$ & 100 & 91.85 & 95.92 \\
\hline 10m_5_70 & 59 & 419.26 & $5.900 \mathrm{E}+06$ & 239.13 & 59 & 263.06 & $5.900 \mathrm{E}+06$ & 75 & 211.27 & 143.13 \\
\hline 101_5_70 & 43 & 541.38 & $4.301 \mathrm{E}+06$ & 292.19 & 43 & 418.37 & $4.300 \mathrm{E}+06$ & 51 & 87.02 & 69.01 \\
\hline 20s_1_30 & 0 & 1221.04 & $1.221 \mathrm{E}+03$ & 610.52 & 0 & 160.38 & $1.603 \mathrm{E}+02$ & - & - & - \\
\hline 20m_1_30 & 0 & 1821.80 & $1.822 \mathrm{E}+03$ & 910.90 & 0 & 225.29 & $2.252 \mathrm{E}+02$ & 1 & 235.90 & 118.45 \\
\hline 201_1_30 & 0 & 2171.42 & $2.171 \mathrm{E}+03$ & 1085.71 & 0 & 198.40 & $1.984 \mathrm{E}+02$ & 0 & 261.18 & 130.59 \\
\hline $20 s \_1 \_50$ & 4 & 1412.59 & $4.014 \mathrm{E}+05$ & 708.30 & 4 & 425.09 & $4.004 \mathrm{E}+02$ & - & - & - \\
\hline 20m_1_50 & 0 & 1373.24 & $1.373 \mathrm{E}+03$ & 686.21 & 0 & 445.37 & $4.453 \mathrm{E}+02$ & 9 & 351.42 & 180.21 \\
\hline 201_1_50 & 0 & 1851.12 & $1.851 \mathrm{E}+03$ & 925.56 & 0 & 155.85 & $1.558 \mathrm{E}+02$ & 8 & 118.23 & 63.11 \\
\hline $20 s \_1 \_70$ & 448 & 801.34 & $4.480 \mathrm{E}+07$ & 624.67 & 448 & 692.26 & $4.480 \mathrm{E}+07$ & 480 & 196.16 & 338.08 \\
\hline 20m_1_70 & 241 & 2396.80 & $2.410 \mathrm{E}+07$ & 1318.90 & 241 & 1287.71 & $2.410 \mathrm{E}+07$ & 333 & 640.11 & 486.55 \\
\hline 201_1_70 & 77 & 1210.96 & $7.701 \mathrm{E}+06$ & 643.98 & 77 & 758.37 & $7.700 \mathrm{E}+06$ & 88 & 337.18 & 212.59 \\
\hline 20s_3_30 & 54 & 1328.04 & $5.401 \mathrm{E}+06$ & 691.02 & - & - & - & - & - & - \\
\hline 20m_3_30 & 995 & 2031.35 & $9.950 \mathrm{E}+07$ & 1513.18 & 15 & 740.32 & $1.500 \mathrm{E}+06$ & 64 & 676.63 & 370.31 \\
\hline 201_3_30 & 648 & - & - & - & 0 & 277.50 & $2.775 \mathrm{E}+02$ & 37 & 417.42 & 227.21 \\
\hline 20s_3_50 & 168 & 1434.26 & $1.680 \mathrm{E}+07$ & 799.13 & - & - & - & - & - & - \\
\hline 20m_3_50 & 99 & 2203.80 & $9.902 \mathrm{E}+06$ & 1151.40 & - & - & - & 181 & 366.17 & 273.58 \\
\hline 201_3_50 & 1344 & 1852.22 & $1.344 \mathrm{E}+08$ & 1598.11 & 45 & 1123.48 & $4.501 \mathrm{E}+06$ & 49 & 578.48 & 313.74 \\
\hline 20s_3_70 & 423 & 1552.35 & $4.230 \mathrm{E}+07$ & 987.67 & 424 & 2902.26 & $4.240 \mathrm{E}+07$ & 528 & 390.82 & 459.41 \\
\hline 20m_3_70 & 168 & 1491.69 & $1.680 \mathrm{E}+07$ & 829.84 & 168 & 1407.24 & $1.680 \mathrm{E}+07$ & - & - & - \\
\hline 201_3_70 & 274 & 2989.96 & $2.740 \mathrm{E}+07$ & 1631.98 & - & - & - & - & - & - \\
\hline 20s_5_30 & 923 & 939.96 & $9.230 \mathrm{E}+07$ & 931.48 & 68 & 1630.26 & $6.801 \mathrm{E}+06$ & - & - & - \\
\hline 20m_5_30 & 886 & - & - & - & 43 & 2017.09 & $4.302 \mathrm{E}+06$ & - & - & - \\
\hline 20s_5_50 & 1527 & 1051.42 & $1.527 \mathrm{E}+08$ & 1289.21 & - & - & - & - & - & - \\
\hline 20m_5_50 & 1450 & - & - & - & 87 & 2062.80 & $8.702 \mathrm{E}+06$ & - & - & - \\
\hline 20s_5_70 & 330 & 1499.62 & $3.300 \mathrm{E}+07$ & 914.81 & - & - & - & - & - & - \\
\hline 20m_5_70 & 249 & 1435.31 & $2.490 \mathrm{E}+07$ & 842.15 & 385 & 1738.69 & $3.850 \mathrm{E}+07$ & - & - & - \\
\hline 201_5_70 & 129 & - & - & - & - & - & - & 158 & 812.23 & 485.11 \\
\hline
\end{tabular}


Table 5: Computational results obtained by solving the mathematical formulations (continued).

\begin{tabular}{|c|c|c|c|c|c|c|c|c|c|c|}
\hline \multirow{2}{*}{ Instance } & \multicolumn{4}{|c|}{$\mathcal{F}_{L A}+\mathcal{F}_{C S}$} & \multicolumn{3}{|c|}{$\mathcal{F}_{C W S}(\alpha>>\beta)$} & \multicolumn{3}{|c|}{$\mathcal{F}_{C W S}(\alpha=\beta)$} \\
\hline & $E_{L A}$ & $E_{C S}$ & $E_{\tau}(\alpha>>\beta)$ & $E_{\tau}(\alpha=\beta)$ & $E_{L A}$ & $E_{C S}$ & $E_{\tau}$ & $E_{L A}$ & $E_{C S}$ & $E_{\tau}$ \\
\hline $30 s_{-} 1 \_30$ & 0 & 3495.86 & $3.496 \mathrm{E}+03$ & 1747.93 & 0 & 668.80 & $6.688 \mathrm{E}+02$ & - & - & - \\
\hline 30m_1_30 & 0 & 4501.77 & $4.502 \mathrm{E}+03$ & 2250.88 & 0 & 921.33 & $9.213 \mathrm{E}+02$ & - & - & - \\
\hline 301_1_30 & $\mathbf{0}$ & 4911.02 & $4.911 \mathrm{E}+03$ & 2455.51 & 0 & 634.49 & $6.344 \mathrm{E}+02$ & - & - & - \\
\hline 30s_1_50 & 98 & 3299.06 & $9.803 \mathrm{E}+06$ & 1698.53 & 98 & 1303.66 & $9.801 \mathrm{E}+06$ & 210 & 729.63 & 469.81 \\
\hline 30m_1_50 & 19 & 3742.17 & $1.904 \mathrm{E}+06$ & 1880.58 & 19 & 2616.36 & $1.902 \mathrm{E}+06$ & 75 & 1880.72 & 977.86 \\
\hline 301_1_50 & 0 & 4683.93 & $4.684 \mathrm{E}+03$ & 2737.80 & 0 & 724.51 & $7.245 \mathrm{E}+02$ & - & - & - \\
\hline $30 s_{-} 1 \_70$ & 934 & 2416.98 & $9.340 \mathrm{E}+07$ & 1675.49 & 937 & 2667.16 & $9.370 \mathrm{E}+07$ & - & - & - \\
\hline 30m_1_70 & 544 & 5919.16 & $5.441 \mathrm{E}+07$ & 3231.58 & 554 & 3078.50 & $5.540 \mathrm{E}+07$ & 631 & 2046.16 & 1338.58 \\
\hline 301_1_70 & 249 & 3310.89 & $2.490 \mathrm{E}+07$ & 1779.94 & - & - & - & 268 & 1623.10 & 945.55 \\
\hline 30s_3_30 & 1585 & 2858.33 & $1.585 \mathrm{E}+08$ & 2221.66 & - & - & - & - & - & - \\
\hline 30s_3_50 & 330 & 3643.15 & $3.300 \mathrm{E}+07$ & 1986.57 & - & - & - & - & - & - \\
\hline 30s_3_70 & 821 & 3058.59 & $8.210 \mathrm{E}+07$ & 1939.79 & 840 & 3613.93 & $8.400 \mathrm{E}+07$ & - & - & - \\
\hline 30m_3_70 & 371 & 3394.37 & $3.710 \mathrm{E}+07$ & 1882.68 & - & - & - & - & - & - \\
\hline 301_3_70 & 2589 & - & - & - & - & - & - & 684 & 9339.08 & 5019.04 \\
\hline 30s_5_70 & 756 & 4492.94 & $7.560 \mathrm{E}+07$ & 2624.47 & - & - & - & - & - & - \\
\hline 50s_1_30 & 0 & 16686.92 & $1.669 \mathrm{E}+04$ & 8343.46 & - & - & - & - & - & - \\
\hline 50m_1_30 & 0 & 17645.35 & $1.765 \mathrm{E}+04$ & 8822.67 & 0 & 8264.20 & $8.264 \mathrm{E}+03$ & - & - & - \\
\hline 50s_1_50 & 401 & 12290.55 & $4.011 \mathrm{E}+07$ & 6345.77 & - & - & - & - & - & - \\
\hline 50m_1_50 & 250 & 13869.28 & $2.501 \mathrm{E}+07$ & 7059.64 & - & - & - & - & - & - \\
\hline 501_1_50 & 11 & 15907.70 & $1.116 \mathrm{E}+06$ & 7959.35 & - & - & - & 146 & 9867.24 & 5006.62 \\
\hline 50s_1_70 & 1743 & 11544.01 & $1.743 \mathrm{E}+08$ & 6643.50 & 1772 & 6898.15 & $1.772 \mathrm{E}+08$ & 2370 & 4006.59 & 3188.29 \\
\hline 50m_1_70 & 1017 & 19637.64 & $1.017 \mathrm{E}+08$ & 10327.32 & - & - & - & - & - & - \\
\hline 501_1_70 & 599 & 20988.08 & $5.992 \mathrm{E}+07$ & 10793.54 & - & - & - & - & - & - \\
\hline 50s_3_70 & 3904 & 12233.50 & $3.904 \mathrm{E}+08$ & 8068.75 & - & - & - & - & - & - \\
\hline 70s_1_30 & 67 & 19600.96 & $6.720 \mathrm{E}+06$ & 9833.98 & - & - & - & - & - & - \\
\hline 70s_1_50 & 1436 & 20060.26 & $1.436 \mathrm{E}+08$ & 10748.13 & - & - & - & - & - & - \\
\hline 70s_1_70 & 3225 & 16797.21 & $3.225 \mathrm{E}+08$ & 10011.10 & - & - & - & - & - & - \\
\hline 70m_1_70 & 2023 & 29211.91 & $2.023 \mathrm{E}+08$ & 15617.45 & - & - & - & - & - & - \\
\hline 701_1_70 & 1332 & 41511.74 & $1.332 \mathrm{E}+08$ & 21421.87 & - & - & - & - & - & - \\
\hline
\end{tabular}

The results indicate that the sequential approach yields better LAP solutions for only five instances, 20m_5_70, 30s_1_70, 30m_1_70, 30s_3_70 and 50s_1_70. This can be explained by the observation that in these two cases, none of the alternative approaches finds an optimal solution. For the remaining instances, the value of $E_{L A}$ obtained by $\mathcal{F}_{C W S}(\alpha>>\beta)$ is better than or equal to the value of $E_{L A}$ obtained by $\mathcal{F}_{L A}+\mathcal{F}_{C S}$, while $\mathcal{F}_{C W S}(\alpha>>\beta)$ always finds a better crane scheduling solution. However, $\mathcal{F}_{C W S}(\alpha>>\beta)$ was only able to find feasible solutions for two large instances (number of requests greater than 50). $\mathcal{F}_{C W S}(\alpha>>\beta)$ may change location assignments and is therefore able to find better crane scheduling while still achieving the same quality of location assignments. This shows how by integrating the LAP and the CSP, better location assignments and crane schedules are achievable. Generating better crane schedules by $\mathcal{F}_{C W S}(\alpha>>\beta)$ may require compromises with respect to computational runtimes. The results show that $\mathcal{F}_{C W S}(\alpha>>\beta)$ tends to take more time to achieve optima for small instances. However for larger instances both approaches take the entire runtime.

When $\alpha$ is equal to $\beta$, it is evident that the $E_{L A}$ values obtained by $\mathcal{F}_{L A}+\mathcal{F}_{C S}$ and $\mathcal{F}_{C W S}(\alpha>>$ $\beta)$ are lower than those obtained by $\mathcal{F}_{C W S}(\alpha=\beta)$. However, $E_{\tau}$ is significantly lower in $\mathcal{F}_{C W S}(\alpha=$ $\beta)$ than in $\mathcal{F}_{L A}+\mathcal{F}_{C S}(\alpha=\beta)$. Although $\mathcal{F}_{C W S}(\alpha>>\beta)$ is capable of finding good crane schedules while achieving high quality location assignments, the $E_{C S}$ obtained by $\mathcal{F}_{C W S}(\alpha=\beta)$ is lower. There are only two instances, 201_3_30 and 201_1_30, where $\mathcal{F}_{C W S}(\alpha>>\beta)$ achieved both better location assignments and crane scheduling within the available runtime. For the remaining instances, $\mathcal{F}_{C S}(\alpha=\beta)$ compromises as regards location assignment to achieve better 
Table 6: Tuning parameters by irace package

\begin{tabular}{|c|c|c|c|c|c|}
\hline \multirow{2}{*}{ Parameter } & \multirow{2}{*}{ Role } & \multirow{2}{*}{ Type } & \multirow{2}{*}{ Range } & \multicolumn{2}{|c|}{ irace results } \\
\hline & & & & integrated & sequential \\
\hline$l_{c w s}$ & $\frac{\text { Size of the late acceptance list for CWSP LAHC }}{\text { number of requests }}$ & integer & $(10,250)$ & 56 & - \\
\hline$l_{l a p}$ & $\frac{\text { Size of the late acceptance list for LAP LAHC }}{\text { number of requests }}$ & integer & $(10,250)$ & - & 174 \\
\hline$l_{c s p}$ & $\frac{\text { Size of the late acceptance list for CSP LAHC }}{\text { number of requests }}$ & integer & $(10,250)$ & - & 104 \\
\hline$p_{l r}$ & Weight of using $L R$ neighbourhood & real & $(0.0,1.0)$ & 0.47 & 0.13 \\
\hline$p_{l s}$ & Weight of using $L S$ neighbourhood & real & $(0.0,1.0)$ & 0.18 & 0.07 \\
\hline$p_{g l a}$ & Weight of using GLA neighbourhood & real & $(0.0,1.0)$ & 0.40 & 0.87 \\
\hline$p_{c r}$ & Weight of using $C R$ neighbourhood & real & $(0.0,1.0)$ & 0.18 & 0.59 \\
\hline$p_{o s}$ & Weight of using $O S$ neighbourhood & real & $(0.0,1.0)$ & 0.34 & 0.36 \\
\hline$p_{r i}$ & Weight of using $R I$ neighbourhood & real & $(0.0,1.0)$ & 0.08 & 0.22 \\
\hline$p_{r b i}$ & Weight of using $R B I$ neighbourhood & real & $(0.0,1.0)$ & 0.47 & 0.64 \\
\hline$p_{n l a}$ & Weight of using $N L A$ neighbourhood & real & $(0.0,1.0)$ & 0.70 & - \\
\hline$p_{\text {bop }}$ & Weight of using $B O P$ neighbourhood & real & $(0.0,1.0)$ & 0.52 & 0.38 \\
\hline$p_{m b o}$ & Weight of using $M B O P$ neighbourhood & real & $(0.0,1.0)$ & 0.07 & 0.18 \\
\hline
\end{tabular}

crane schedules. The number of instances solved to optimality decreases as the number of requests grows.

The detailed table of results including the optimality gap and computation time $(c p u)$ of both approaches is presented in the Appendix (Table A.8).

\subsection{Comparison of sequential and integrated approaches by the proposed heuristics}

The heuristic was implemented in $\mathrm{C}++11$. All experiments were executed for maximum 300 seconds or maximum 10,000 consecutive iterations without improvement for all 135 instances. The heuristic's parameters were tuned using the irace R-package which implements the iterated racing procedure for automatic algorithm configuration (López-Ibáñez et al., 2016) with a budget of 4,000 runs. The purpose of irace is to automate the task of configuring an optimization algorithm's parameters. It generates and tests a sample of parameter configurations for a given optimization algorithm on a set of instances. When sufficient statistical evidence is collected (by means of a Friedman test) that a certain parameter configuration is outperformed by others, it is discarded so as to focus on the remaining configurations. The best-performing configurations are reported.

Table 6 summarizes the parameters by their role, type, range and tuned values for both approaches (sequential and integrated).

Table 7 reports the results of the three heuristics when the values of $\alpha$ and $\beta$ are equal to 0.5 ( $\alpha=\beta=0.5$ ). The location assignment evaluation $\left(E_{L A}\right)$, crane scheduling evaluation $\left(E_{C S}\right)$ and total cost $\left(E_{\tau}\right)$ obtained by the three heuristics are compared. The heuristic was run five times with different random seeds, and the average of these results is reported. The improvement of the integrated and sequential heuristics over the constructive heuristic $(\mathrm{G})$, the upper bound (UB) defined as the best feasible solution obtained by either the sequential or integrated formulation, and the relative optimality gap (gap) and the computational times $(c p u)$ are reported. The optimality gap is measured by comparing $E_{\tau}$ obtained by the integrated heuristic and the lower bound generated by the mathematical formulations. The gaps may be relatively large as the lower bounds 
are weak due to employing large numbers $\left(M_{L}\right.$ and $\left.M_{T}\right)$ in the mathematical formulations. When $\mathcal{F}_{C W S}(\alpha=\beta)$ was unable to find a lower bound due to an out-of-memory error, OOM is reported. Emboldened numbers indicate that optimum solutions were obtained.

The results show how the integrated heuristics find high quality solutions and are even able to find optimum solutions for the instances with ten requests and stacking level equal to one, in a short amount of time. There are only two instances $10 \mathrm{~s}_{-} 1 \_70$ and $10 \mathrm{~m}_{-} 1 \_70$ where the integrated heuristic was unable to find the optimum solution. Nevertheless, the optimality gaps are only $1.1 \%$ and $4.8 \%$, respectively. There are instances for which the sequential heuristic finds better solutions than the optimum obtained by $\mathcal{F}_{L A}+\mathcal{F}_{C S}$. The explanation for these interesting results lies in how the sequential heuristic finds location assignments which enable better crane scheduling. 
Table 7: Computational results of Constructive, Sequential and Integrated heuristics $(\alpha=\beta=0.5)$

\begin{tabular}{|c|c|c|c|c|c|c|c|c|c|c|c|c|c|c|c|c|}
\hline \multirow{2}{*}{ istance } & \multicolumn{3}{|c|}{ Dispatching rules } & \multicolumn{5}{|c|}{ Sequential } & \multicolumn{6}{|c|}{ Integrated } & \multicolumn{2}{|c|}{ Best MIP bounds } \\
\hline & $E_{L A}$ & $E_{C S}$ & $E_{\tau}$ & $E_{L A}$ & $E_{C S}$ & $E_{\tau}$ & cpu & $\mathrm{G} \%$ & $E_{L A}$ & $E_{C S}$ & $E_{\tau}$ & сри & $\mathrm{G} \%$ & gap & UB & LB \\
\hline $10 s \_1 \_30$ & 0 & 108.0 & 54.0 & 0 & 59.9 & 30.0 & 3.7 & 44.5 & 1 & 51.5 & 26.3 & 4.9 & 51.4 & 0.0 & 26.3 & 26.3 \\
\hline 10m_1_30 & 0 & 234.1 & 117.1 & 0 & 37.6 & 18.8 & 6.1 & 83.9 & 0 & 37.6 & 18.8 & 3.5 & 83.9 & 0.0 & 18.8 & 18.8 \\
\hline 101_1_30 & 0 & 384.0 & 192.0 & 0 & 79.8 & 39.9 & 10.5 & 79.2 & 0 & 76.8 & 38.4 & 6.5 & 80.0 & 0.0 & 38.4 & 38.4 \\
\hline 10 s_1_50 & 0 & 235.8 & 117.9 & 0 & 137.9 & 69.0 & 4.2 & 41.5 & 11 & 79.0 & 45.0 & 9.2 & 61.8 & 0.0 & 45.0 & 45.0 \\
\hline 10m_1_50 & 0 & 366.8 & 183.4 & 0 & 96.3 & 48.1 & 7.7 & 73.8 & 7 & 40.1 & 23.5 & 7.8 & 87.2 & 0.0 & 23.5 & 23.5 \\
\hline 101_1_50 & 0 & 350.4 & 175.2 & 0 & 45.4 & 22.7 & 13.0 & 87.0 & 1 & 35.5 & 18.2 & 8.1 & 89.6 & 0.0 & 18.2 & 18.2 \\
\hline $10 \mathrm{~s} \_1 \_70$ & 180 & 767.9 & 474.0 & 132 & 158.4 & 145.2 & 4.4 & 69.4 & 150 & 67.7 & 108.8 & 4.8 & 77.0 & 1.1 & 107.6 & 107.6 \\
\hline 10m_1_70 & 48 & 1031.6 & 539.8 & 48 & 405.8 & 226.9 & 9.1 & 58.0 & 86 & 199.8 & 142.9 & 10.2 & 73.5 & 4.8 & 136.0 & 136.0 \\
\hline 101_1_70 & 11 & 843.8 & 427.4 & 11 & 166.1 & 88.5 & 14.9 & 79.3 & 25 & 73.8 & 49.4 & 29.3 & 88.4 & 0.0 & 49.4 & 49.4 \\
\hline 10 s_3_30 & 13 & 324.8 & 168.9 & 13 & 230.8 & 121.9 & 9.0 & 27.8 & 50 & 86.1 & 68.0 & 13.4 & 59.7 & 40.8 & 62.5 & 40.3 \\
\hline 10m_3_30 & 1 & 400.8 & 200.9 & 1 & 185.9 & 93.5 & 17.8 & 53.5 & 6 & 76.4 & 41.2 & 24.8 & 79.5 & 81.1 & 39.7 & 7.8 \\
\hline 101_3_30 & 0 & 700.8 & 350.4 & 0 & 98.5 & 49.3 & 32.2 & 85.9 & 3 & 60.3 & 31.7 & 22.1 & 91.0 & 78.2 & 147.6 & 6.9 \\
\hline $10 s_{3} 3 \_50$ & 58 & 538.0 & 298.0 & 53 & 275.2 & 164.1 & 11.1 & 44.9 & 60 & 111.5 & 85.8 & 12.9 & 71.2 & 48.8 & 86.9 & 43.9 \\
\hline 10m_3_50 & 68 & 686.9 & 377.4 & 31 & 333.3 & 182.2 & 24.8 & 51.7 & 42 & 90.6 & 66.3 & 54.1 & 82.4 & 75.6 & 83.9 & 16.2 \\
\hline 101_3_50 & 9 & 1181.3 & 595.2 & 9 & 258.0 & 133.5 & 48.4 & 77.6 & 12 & 85.9 & 49.0 & 82.9 & 91.8 & 0.0 & 49.0 & 49.0 \\
\hline 10 s_3_70 & 150 & 1309.0 & 729.5 & 130 & 304.4 & 217.2 & 12.0 & 70.2 & 168 & 117.4 & 142.7 & 8.1 & 80.4 & 36.9 & 146.2 & 90.0 \\
\hline 10m_3_70 & 64 & 1210.5 & 637.2 & 64 & 273.8 & 168.9 & 25.5 & 73.5 & 79 & 104.8 & 91.9 & 69.0 & 85.6 & 26.3 & 85.5 & 67.7 \\
\hline 101_3_70 & 106 & 697.3 & 401.7 & 98 & 321.4 & 209.7 & 57.2 & 47.8 & 115 & 112.8 & 113.9 & 102.3 & 71.6 & 50.4 & 126.1 & 56.5 \\
\hline 10 s_5_30 & 18 & 509.4 & 263.7 & 18 & 201.5 & 109.8 & 36.6 & 58.4 & 47 & 84.7 & 65.9 & 25.9 & 75.0 & 67.2 & 64.1 & 21.6 \\
\hline 10m_5_30 & 9 & 972.4 & 490.7 & 9 & 372.8 & 190.9 & 32.2 & 61.1 & 29 & 66.8 & 47.9 & 47.2 & 90.2 & 77.5 & 50.2 & 10.8 \\
\hline 101_5_30 & 6 & 988.0 & 497.0 & 6 & 417.5 & 211.7 & 85.0 & 57.4 & 18 & 95.1 & 56.6 & 137.1 & 88.6 & 70.6 & 111.4 & 16.6 \\
\hline $10 s \_5 \_50$ & 66 & 717.0 & 391.5 & 66 & 341.7 & 203.8 & 23.1 & 47.9 & 71 & 123.9 & 97.5 & 22.4 & 75.1 & 56.6 & 250.8 & 42.3 \\
\hline 10m_5_50 & 91 & 619.3 & 355.1 & 26 & 405.8 & 215.9 & 51.3 & 39.2 & 59 & 149.6 & 104.3 & 80.7 & 70.6 & 85.0 & 119.3 & 15.6 \\
\hline 101_5_50 & 51 & 517.4 & 284.2 & 13 & 199.3 & 106.1 & 59.8 & 62.7 & 19 & 85.1 & 52.1 & 105.1 & 81.7 & 56.2 & 52.0 & 22.8 \\
\hline 10 s_5_70 & 89 & 374.7 & 231.9 & 83 & 272.6 & 177.8 & 18.1 & 23.3 & 100 & 91.9 & 95.9 & 12.9 & 58.6 & 32.3 & 95.9 & 64.9 \\
\hline 10m_5_70 & 84 & 1009.5 & 546.7 & 59 & 427.5 & 243.2 & 41.2 & 55.5 & 75 & 183.2 & 129.1 & 120.3 & 76.4 & 76.6 & 143.1 & 30.2 \\
\hline 101_5_70 & 44 & 1426.0 & 735.0 & 44 & 354.8 & 199.4 & 85.6 & 72.9 & 49 & 98.2 & 73.6 & 82.7 & 90.0 & 33.7 & 69.0 & 48.8 \\
\hline $20 s \_1 \_30$ & 0 & 358.9 & 179.4 & 0 & 190.5 & 95.2 & 14.8 & 46.9 & 18 & 134.7 & 76.4 & 42.2 & 57.4 & 62.8 & 610.5 & 28.4 \\
\hline 20m_1_30 & 0 & 854.5 & 427.2 & 0 & 243.9 & 122.0 & 25.3 & 71.5 & 10 & 200.4 & 105.2 & 38.8 & 75.4 & 83.0 & 118.5 & 17.9 \\
\hline 201_1_30 & 0 & 2741.4 & 1370.7 & 0 & 205.4 & 102.7 & 51.5 & 92.5 & 0 & 195.2 & 97.6 & 269.5 & 92.9 & 67.5 & 130.6 & 31.7 \\
\hline 20 s_1 1 -50 & 4 & 1652.1 & 828.1 & 4 & 539.1 & 271.5 & 21.8 & 67.2 & 44 & 198.5 & 121.3 & 54.8 & 85.4 & 71.0 & 708.3 & 35.2 \\
\hline 20m_1_50 & 0 & 1556.1 & 778.1 & 0 & 467.9 & 233.9 & 32.0 & 69.9 & 28 & 263.9 & 146.0 & 74.2 & 81.2 & 88.2 & 180.2 & 17.2 \\
\hline 201_1_50 & 0 & 904.6 & 452.3 & 0 & 185.7 & 92.9 & 45.4 & 79.5 & 4 & 134.9 & 69.4 & 113.3 & 84.7 & 94.2 & 63.1 & 4.0 \\
\hline 20 s_1_70 & 550 & 2862.2 & 1706.1 & 448 & 629.7 & 538.8 & 24.3 & 68.4 & 485 & 186.1 & 335.6 & 37.8 & 80.3 & 29.5 & 338.1 & 236.6 \\
\hline 20m_1_70 & 258 & 4543.9 & 2401.0 & 242 & 1188.2 & 715.1 & 38.6 & 70.2 & 380 & 402.6 & 391.3 & 47.8 & 83.7 & 62.4 & 486.6 & 147.1 \\
\hline 201_1_70 & 77 & 3110.3 & 1593.6 & 77 & 726.1 & 401.5 & 63.3 & 74.8 & 91 & 241.1 & 166.1 & 113.6 & 89.6 & 61.0 & 212.6 & 64.7 \\
\hline 20 s_3_30 & 100 & 2680.3 & 1390.1 & 54 & 902.8 & 478.4 & 30.1 & 65.6 & 133 & 163.8 & 148.4 & 104.3 & 89.3 & 79.7 & 691.0 & 30.1 \\
\hline 20m_3_30 & 15 & 2364.1 & 1189.6 & 15 & 826.4 & 420.7 & 89.4 & 64.6 & 99 & 273.0 & 186.0 & 159.1 & 84.4 & 89.9 & 370.3 & 18.7 \\
\hline 201_3_30 & 0 & 2033.1 & 1016.6 & 0 & 307.8 & 153.9 & 98.8 & 84.9 & 35 & 148.0 & 91.5 & 168.2 & 91.0 & 92.0 & 227.2 & 7.3 \\
\hline 20 s_3_50 & 225 & 1467.3 & 846.1 & 139 & 603.1 & 371.0 & 36.1 & 56.2 & 174 & 313.4 & 243.7 & 89.4 & 71.2 & 75.9 & 799.1 & 58.8 \\
\hline 20m_3_50 & 130 & 3485.8 & 1807.9 & 97 & 1162.3 & 629.6 & 78.0 & 65.2 & 193 & 257.8 & 225.4 & 197.9 & 87.5 & 86.4 & 273.6 & 30.7 \\
\hline 201_3_50 & 45 & 4481.3 & 2263.1 & 45 & 839.2 & 442.1 & 161.9 & 80.5 & 90 & 151.2 & 120.6 & 300.0 & 94.7 & 74.9 & 313.7 & 30.3 \\
\hline 20 s_3_70 & 543 & 5436.3 & 2989.6 & 438 & 1000.6 & 719.3 & 106.4 & 75.9 & 493 & 210.1 & 351.5 & 102.2 & 88.2 & 42.3 & 459.4 & 202.8 \\
\hline
\end{tabular}


Table 7: Computational results of Dispatching rules, Sequential and Integrated heuristics (continued)

\begin{tabular}{|c|c|c|c|c|c|c|c|c|c|c|c|c|c|c|c|c|}
\hline \multirow[b]{2}{*}{ istance } & \multicolumn{3}{|c|}{ Dispatching rules } & \multicolumn{5}{|c|}{ Sequential } & \multicolumn{6}{|c|}{ Integrated } & \multicolumn{2}{|c|}{ Best MIP bounds } \\
\hline & $E_{L A}$ & $E_{C S}$ & $E_{\tau}$ & $E_{L A}$ & $E_{C S}$ & $E_{\tau}$ & cpu & $\mathrm{G} \%$ & $E_{L A}$ & $E_{C S}$ & $E_{\tau}$ & cpu & G\% & gap & UB & $\mathrm{LB}$ \\
\hline 20m_3_70 & 197 & 4097.8 & 2147.4 & 170 & 1308.0 & 739.0 & 104.8 & 65.6 & 210 & 361.5 & 285.7 & 163.6 & 86.7 & 72.5 & 829.8 & 78.5 \\
\hline 201_3_70 & 307 & 3384.8 & 1845.9 & 275 & 1637.5 & 956.2 & 160.0 & 48.2 & 335 & 250.5 & 292.7 & 300.0 & 84.1 & 65.2 & 1632.0 & 101.8 \\
\hline 20 s_5_30 & 80 & 2458.7 & 1269.4 & 73 & 865.1 & 469.0 & 48.6 & 63.0 & 127 & 273.4 & 200.2 & 99.6 & 84.2 & 88.0 & 931.5 & 24.0 \\
\hline 20m_5_30 & 37 & 2977.7 & 1507.3 & 37 & 1041.4 & 539.2 & 163.6 & 64.2 & 130 & 260.8 & 195.4 & 129.8 & 87.0 & 90.0 & - & 19.5 \\
\hline 201_5_30 & 30 & 3035.3 & 1532.6 & 30 & 850.9 & 440.5 & 212.7 & 71.3 & 80 & 317.1 & 198.5 & 299.6 & 87.0 & - & - & $\mathrm{OOM}$ \\
\hline 20s_5_50 & 210 & 2552.3 & 1381.1 & 194 & 895.0 & 544.5 & 71.2 & 60.6 & 213 & 266.6 & 239.8 & 133.0 & 82.6 & 79.8 & 1289.2 & 48.4 \\
\hline 20m_5_50 & 192 & 2472.6 & 1332.3 & 83 & 1078.2 & 580.6 & 210.9 & 56.4 & 298 & 355.1 & 326.5 & 300.0 & 75.5 & - & - & OOM \\
\hline 201_5_50 & 146 & 2917.4 & 1531.7 & 68 & 953.2 & 510.6 & 201.2 & 66.7 & 81 & 287.6 & 184.3 & 300.0 & 88.0 & - & - & $\mathrm{OOM}$ \\
\hline 20s_5_70 & 390 & 3077.3 & 1733.6 & 321 & 933.3 & 627.1 & 79.1 & 63.8 & 465 & 282.9 & 373.9 & 156.4 & 78.4 & 73.0 & 914.8 & 100.8 \\
\hline 20m_5_70 & 350 & 4295.8 & 2322.9 & 252 & 1521.8 & 886.9 & 156.2 & 61.8 & 321 & 496.2 & 408.6 & 283.6 & 82.4 & 85.2 & 842.2 & 60.6 \\
\hline 201_5_70 & 130 & 5138.0 & 2634.0 & 123 & 1035.4 & 579.2 & 176.2 & 78.0 & 224 & 334.3 & 279.1 & 300.0 & 89.4 & 81.5 & 485.1 & 51.7 \\
\hline $30 s \_1 \_30$ & 0 & 1777.2 & 888.6 & 0 & 447.0 & 223.5 & 53.0 & 74.8 & 35 & 283.2 & 159.1 & 126.8 & 82.1 & - & 1747.9 & 19.7 \\
\hline 30m_1_30 & 0 & 2025.8 & 1012.9 & 0 & 630.3 & 315.2 & 49.1 & 68.9 & 41 & 407.0 & 224.0 & 115.0 & 77.9 & - & 2250.9 & 26.8 \\
\hline 301_1_30 & 0 & 6808.6 & 3404.3 & 0 & 348.5 & 174.3 & 115.5 & 94.9 & 0 & 337.3 & 168.6 & 178.8 & 95.0 & - & 2455.5 & 24.2 \\
\hline $30 s \_1 \_50$ & 113 & 4217.1 & 2165.0 & 104 & 1353.9 & 729.0 & 63.1 & 66.3 & 140 & 458.9 & 299.4 & 114.4 & 86.2 & - & 469.8 & 67.2 \\
\hline 30m_1_50 & 52 & 4486.5 & 2269.2 & 19 & 2011.0 & 1015.0 & 232.2 & 55.3 & 137 & 492.1 & 314.5 & 244.1 & 86.1 & - & 977.9 & 21.6 \\
\hline 301_1_50 & 7 & 2601.7 & 1304.3 & 0 & 1459.3 & 729.6 & 273.2 & 44.1 & 9 & 280.8 & 144.9 & 268.9 & 88.9 & - & 2737.8 & 13.9 \\
\hline $30 s \_1 \_70$ & 1121 & 6534.2 & 3827.6 & 934 & 1450.6 & 1192.3 & 59.5 & 68.8 & 1034 & 345.9 & 689.9 & 109.7 & 82.0 & - & 1675.5 & 43.8 \\
\hline $30 \mathrm{~m} \_1 \_70$ & 616 & 9826.3 & 5221.1 & 548 & 2912.2 & 1730.1 & 153.2 & 66.9 & 777 & 628.3 & 702.7 & 246.5 & 86.5 & 58.1 & 1338.6 & 294.2 \\
\hline 301_1_70 & 287 & 7409.5 & 3848.2 & 249 & 1572.4 & 910.7 & 119.3 & 76.3 & 260 & 390.4 & 325.2 & 300.0 & 91.5 & 57.5 & 945.6 & 138.1 \\
\hline 30s_3_30 & 173 & 5440.2 & 2806.6 & 132 & 1642.9 & 887.4 & 115.0 & 68.4 & 217 & 345.6 & 281.3 & 300.0 & 90.0 & - & 2221.7 & 37.0 \\
\hline 30m_3_30 & 59 & 5332.9 & 2695.9 & 59 & 2237.0 & 1148.0 & 161.4 & 57.4 & 275 & 464.0 & 369.5 & 300.0 & 86.3 & - & - & OOM \\
\hline 301_3_30 & 35 & 3494.1 & 1764.5 & 12 & 1387.4 & 699.7 & 300.0 & 60.3 & 66 & 257.7 & 161.9 & 300.0 & 90.8 & - & - & OOM \\
\hline 30 s_3_50 & 414 & 4759.2 & 2586.6 & 320 & 1649.5 & 984.7 & 96.5 & 61.9 & 419 & 506.4 & 462.7 & 300.0 & 82.1 & - & 1986.6 & OOM \\
\hline 30m_3_50 & 234 & 8504.2 & 4369.1 & 199 & 2816.5 & 1507.7 & 186.2 & 65.5 & 470 & 412.1 & 441.1 & 300.0 & 89.9 & - & - & $\mathrm{OOM}$ \\
\hline 301_3_50 & 115 & 9105.0 & 4610.0 & 112 & 2358.9 & 1235.5 & 186.0 & 73.2 & 206 & 509.7 & 357.9 & 300.0 & 92.2 & - & - & $\mathrm{OOM}$ \\
\hline $30 s \_3 \_70$ & 1080 & 11147.2 & 6113.6 & 918 & 2047.8 & 1482.9 & 172.0 & 75.7 & 913 & 439.3 & 676.2 & 271.5 & 88.9 & 55.5 & 1939.8 & 301.1 \\
\hline 30m_3_70 & 408 & 10669.2 & 5538.6 & 364 & 2798.3 & 1581.1 & 163.7 & 71.5 & 602 & 721.7 & 661.9 & 300.0 & 88.0 & 80.2 & 1882.7 & 130.8 \\
\hline 301_3_70 & 630 & 11675.3 & 6152.7 & 529 & 3183.8 & 1856.4 & 293.6 & 69.8 & 939 & 543.4 & 741.2 & 300.0 & 88.0 & - & 5019.0 & 97.4 \\
\hline 30 s_5_30 & 193 & 5631.7 & 2912.3 & 160 & 1857.8 & 1008.9 & 169.6 & 65.4 & 249 & 561.0 & 405.0 & 176.3 & 86.1 & - & - & OOM \\
\hline 30m_5_30 & 92 & 8734.2 & 4413.1 & 92 & 2129.0 & 1110.5 & 249.6 & 74.8 & 248 & 600.1 & 424.1 & 300.0 & 90.4 & - & - & OOM \\
\hline 301_5_30 & 61 & 6883.5 & 3472.3 & 61 & 1496.6 & 778.8 & 300.0 & 77.6 & 282 & 642.0 & 462.0 & 300.0 & 86.7 & - & - & $\mathrm{OOM}$ \\
\hline 30 s_5_50 & 391 & 7790.2 & 4090.6 & 349 & 2462.8 & 1405.9 & 125.9 & 65.6 & 531 & 408.8 & 469.9 & 300.0 & 88.5 & - & - & 61.0 \\
\hline 30m_5_50 & 275 & 6811.0 & 3543.0 & 166 & 2745.7 & 1455.9 & 252.7 & 58.9 & 665 & 669.1 & 667.0 & 300.0 & 81.2 & - & - & OOM \\
\hline 301_5_50 & 246 & 10671.6 & 5458.8 & 162 & 2531.2 & 1346.6 & 300.0 & 75.3 & 566 & 601.5 & 583.8 & 300.0 & 89.3 & - & - & OOM \\
\hline 30 s_5_70 & 925 & 8077.2 & 4501.1 & 626 & 2258.4 & 1442.2 & 181.2 & 68.0 & 796 & 758.7 & 777.4 & 300.0 & 82.7 & 79.6 & 2624.5 & 158.7 \\
\hline 30m_5_70 & 679 & 8864.2 & 4771.6 & 537 & 3187.9 & 1862.5 & 212.3 & 61.0 & 1152 & 914.8 & 1033.4 & 300.0 & 78.3 & - & - & OOM \\
\hline 301_5_70 & 262 & 10267.7 & 5264.8 & 252 & 2852.5 & 1552.3 & 223.7 & 70.5 & 722 & 715.0 & 718.5 & 300.0 & 86.4 & - & - & $\mathrm{OOM}$ \\
\hline $50 s \_1 \_30$ & 0 & 8471.2 & 4235.6 & 0 & 2143.5 & 1071.8 & 203.5 & 74.7 & 119 & 1139.1 & 629.0 & 226.7 & 85.1 & 89.4 & 8343.5 & 66.5 \\
\hline 50m_1_30 & 0 & 9120.2 & 4560.1 & 0 & 2177.5 & 1088.8 & 199.3 & 76.1 & 139 & 1470.3 & 804.6 & 281.0 & 82.4 & 93.0 & 8822.7 & 56.7 \\
\hline 501_1_30 & 0 & 23604.5 & 11802.2 & 0 & 1661.7 & 830.8 & 225.1 & 93.0 & 4 & 1536.6 & 770.3 & 219.7 & 93.5 & - & - & $\mathrm{OOM}$ \\
\hline 50 s_1_50 & 582 & 16480.0 & 8531.0 & 381 & 4641.6 & 2511.3 & 211.6 & 70.6 & 612 & 1190.7 & 901.4 & 257.3 & 89.4 & 77.9 & 6345.8 & 198.9 \\
\hline
\end{tabular}

(continued on next page) 
Table 7: Computational results of Dispatching rules, Sequential and Integrated heuristics (continued)

\begin{tabular}{|c|c|c|c|c|c|c|c|c|c|c|c|c|c|c|c|c|}
\hline \multirow[b]{2}{*}{ Instance } & \multicolumn{3}{|c|}{ Dispatching rules } & \multicolumn{5}{|c|}{ Sequential } & \multicolumn{6}{|c|}{ Integrated } & \multicolumn{2}{|c|}{ Best MIP bounds } \\
\hline & $E_{L A}$ & $E_{C S}$ & $E_{\tau}$ & $E_{L A}$ & $E_{C S}$ & $E_{\tau}$ & сри & G\% & $E_{L A}$ & $E_{C S}$ & $E_{\tau}$ & сри & G\% & gap & UB & LB \\
\hline 50m_1_50 & 161 & 15745.8 & 7953.4 & 113 & 6542.7 & 3327.9 & 202.5 & 58.2 & 632 & 1854.1 & 1243.0 & 300.0 & 84.4 & 93.3 & 7059.6 & 83.0 \\
\hline 501_1_50 & 39 & 14297.6 & 7168.3 & 12 & 6846.5 & 3429.3 & 300.0 & 52.2 & 151 & 1253.0 & 702.0 & 300.0 & 90.2 & 92.7 & 5006.6 & 51.1 \\
\hline 50 s_1_70 & 2128 & 16748.6 & 9438.3 & 1739 & 4143.1 & 2941.0 & 300.0 & 68.8 & 1993 & 1059.3 & 1526.2 & 239.6 & 83.8 & 47.8 & 3188.3 & 796.8 \\
\hline 50m_1_70 & 1092 & 28900.6 & 14996.3 & 1001 & 7380.2 & 4190.6 & 300.0 & 72.1 & 1747 & 1608.0 & 1677.5 & 286.0 & 88.8 & 69.4 & 10327.3 & 513.7 \\
\hline 501_1_70 & 677 & 25874.3 & 13275.7 & 587 & 7885.2 & 4236.1 & 300.0 & 68.1 & 1044 & 1152.0 & 1098.0 & 300.0 & 91.7 & 71.7 & 10793.5 & 311.1 \\
\hline 50 s_3_30 & 402 & 15748.2 & 8075.1 & 294 & 5025.6 & 2659.8 & 300.0 & 67.1 & 608 & 1131.4 & 869.7 & 300.0 & 89.2 & - & - & OOM \\
\hline 50m_3_30 & 196 & 20272.9 & 10234.5 & 148 & 7168.1 & 3658.0 & 300.0 & 64.3 & 861 & 1522.7 & 1191.8 & 300.0 & 88.4 & - & - & OOM \\
\hline 501_3_30 & 131 & 18198.4 & 9164.7 & 54 & 10340.5 & 5197.3 & 300.0 & 43.3 & 962 & 1361.9 & 1161.9 & 300.0 & 87.3 & - & - & $\mathrm{OOM}$ \\
\hline $50 s \_3 \_50$ & 858 & 18143.2 & 9500.6 & 667 & 5016.4 & 2841.7 & 218.8 & 70.1 & 1237 & 1272.3 & 1254.7 & 300.0 & 86.8 & 89.4 & - & 133.4 \\
\hline 50m_3_50 & 531 & 26273.5 & 13402.3 & 446 & 9864.5 & 5155.3 & 300.0 & 61.5 & 1804 & 1166.2 & 1485.1 & 300.0 & 88.9 & - & - & OOM \\
\hline 501_3_50 & 286 & 30411.5 & 15348.8 & 264 & 10482.6 & 5373.3 & 300.0 & 65.0 & 1785 & 1604.1 & 1694.6 & 300.0 & 89.0 & - & - & OOM \\
\hline 50 s_3_70 & 2236 & 22187.2 & 12211.6 & 1920 & 6035.7 & 3977.8 & 300.0 & 67.4 & 2314 & 1067.4 & 1690.7 & 300.0 & 86.2 & 66.6 & 8068.8 & 564.4 \\
\hline 50m_3_70 & 1072 & 25060.5 & 13066.3 & 870 & 9320.0 & 5095.0 & 300.0 & 61.0 & 2608 & 1606.4 & 2107.2 & 300.0 & 83.9 & - & - & OOM \\
\hline 501_3_70 & 1289 & 41972.4 & 21630.7 & 1106 & 13399.3 & 7252.7 & 300.0 & 66.5 & 3839 & 2053.0 & 2946.0 & 300.0 & 86.4 & - & - & OOM \\
\hline $50 s \_5 \_30$ & 617 & 17668.2 & 9142.6 & 515 & 5204.4 & 2859.7 & 300.0 & 68.7 & 1190 & 1405.5 & 1297.7 & 300.0 & 85.8 & - & - & OOM \\
\hline 50m_5_30 & 346 & 36127.5 & 18236.8 & 346 & 9294.6 & 4820.3 & 300.0 & 73.6 & 1881 & 1872.6 & 1876.8 & 300.0 & 89.7 & - & - & OOM \\
\hline 501_5_30 & 162 & 25689.1 & 12925.5 & 124 & 10134.2 & 5129.1 & 300.0 & 60.3 & 1904 & 1982.9 & 1943.4 & 300.0 & 85.0 & - & - & OOM \\
\hline 50s_5_50 & 1319 & 19176.2 & 10247.6 & 1291 & 8511.1 & 4901.0 & 300.0 & 52.2 & 3012 & 1679.5 & 2345.7 & 300.0 & 77.1 & - & - & OOM \\
\hline 50m_5_50 & 543 & 24680.5 & 12611.8 & 417 & 9208.6 & 4812.8 & 300.0 & 61.8 & 2722 & 2023.6 & 2372.8 & 300.0 & 81.2 & - & - & OOM \\
\hline 501_5_50 & 469 & 37730.4 & 19099.7 & 349 & 13127.9 & 6738.5 & 300.0 & 64.7 & 2327 & 2723.1 & 2525.0 & 300.0 & 86.8 & - & - & OOM \\
\hline 50s_5_70 & 2129 & 21907.9 & 12018.5 & 1689 & 7782.4 & 4735.7 & 300.0 & 60.6 & 3485 & 1736.3 & 2610.6 & 300.0 & 78.3 & - & - & OOM \\
\hline 50m_5_70 & 1803 & 29442.5 & 15622.8 & 1584 & 13188.9 & 7386.5 & 300.0 & 52.7 & 4126 & 4085.9 & 4105.9 & 300.0 & 73.7 & - & - & OOM \\
\hline 501_5_70 & 683 & 35730.4 & 18206.7 & 639 & 16705.5 & 8672.2 & 300.0 & 52.4 & 4363 & 3092.1 & 3727.5 & 300.0 & 79.5 & - & - & $\mathrm{OOM}$ \\
\hline 70s_1_30 & 159 & 21129.6 & 10644.3 & 59 & 9551.3 & 4805.1 & 300.0 & 54.9 & 855 & 2178.6 & 1516.8 & 300.0 & 85.8 & 94.7 & 9834.0 & 80.5 \\
\hline 70m_1_30 & 0 & 20434.8 & 10217.4 & 0 & 4781.7 & 2390.8 & 300.0 & 76.6 & 493 & 2921.1 & 1707.0 & 300.0 & 83.3 & - & - & OOM \\
\hline 701_1_30 & 0 & 40968.3 & 20484.2 & 0 & 4610.7 & 2305.4 & 300.0 & 88.7 & 299 & 2930.8 & 1614.9 & 300.0 & 92.1 & - & - & OOM \\
\hline $70 s \_1 \_50$ & 1618 & 36220.8 & 18919.4 & 1122 & 10137.3 & 5629.7 & 300.0 & 70.2 & 2174 & 2275.6 & 2224.8 & 300.0 & 88.2 & 79.9 & 10748.1 & 447.7 \\
\hline 70m_1_50 & 527 & 35398.5 & 17962.7 & 421 & 14808.9 & 7614.9 & 300.0 & 57.6 & 2503 & 3011.1 & 2757.0 & 300.0 & 84.7 & 93.3 & - & 185.9 \\
\hline 701_1_50 & 277 & 40254.4 & 20265.7 & 119 & 19999.4 & 10059.2 & 300.0 & 50.4 & 1565 & 2625.1 & 2095.1 & 300.0 & 89.7 & - & - & $\mathrm{OOM}$ \\
\hline $70 s \_1 \_70$ & 3854 & 34408.4 & 19131.2 & 3126 & 9247.3 & 6186.6 & 300.0 & 67.7 & 3727 & 2134.4 & 2930.7 & 300.0 & 84.7 & 53.4 & 10011.1 & 1366.7 \\
\hline 70m_1_70 & 2222 & 56536.3 & 29379.1 & 1917 & 17887.0 & 9902.0 & 300.0 & 66.3 & 3786 & 3344.4 & 3565.2 & 300.0 & 87.9 & 74.8 & 15617.5 & 898.4 \\
\hline 701_1_70 & 1339 & 58128.2 & 29733.6 & 1216 & 19929.1 & 10572.6 & 300.0 & 64.4 & 3041 & 2238.2 & 2639.6 & 300.0 & 91.1 & 78.2 & 21421.9 & 575.7 \\
\hline 70s_3_30 & 925 & 30533.0 & 15729.0 & 692 & 12187.0 & 6439.5 & 300.0 & 59.1 & 2229 & 1611.1 & 1920.1 & 300.0 & 87.8 & - & - & OOM \\
\hline 70m_3_30 & 485 & 46373.1 & 23429.1 & 485 & 15703.6 & 8094.3 & 300.0 & 65.5 & 2383 & 2675.3 & 2529.1 & 300.0 & 89.2 & - & - & OOM \\
\hline 701_3_30 & 263 & 38437.7 & 19350.3 & 175 & 26446.0 & 13310.5 & 300.0 & 31.2 & 2363 & 4207.5 & 3285.2 & 300.0 & 83.0 & - & - & OOM \\
\hline 70s_3_50 & 1653 & 40538.0 & 21095.5 & 1220 & 12007.4 & 6613.7 & 300.0 & 68.6 & 2837 & 1971.9 & 2404.4 & 300.0 & 88.6 & - & - & OOM \\
\hline 70m_3_50 & 4039 & 52832.0 & 28435.5 & 973 & 16526.0 & 8749.5 & 300.0 & 69.2 & 4608 & 2439.0 & 3523.5 & 300.0 & 87.6 & - & - & OOM \\
\hline 701_3_50 & 656 & 64629.5 & 32642.8 & 561 & 26877.7 & 13719.4 & 300.0 & 58.0 & 4183 & 3868.6 & 4025.8 & 300.0 & 87.7 & - & - & OOM \\
\hline 70 s_3_70 & 994 & 47335.1 & 24164.6 & 3387 & 13763.5 & 8575.2 & 300.0 & 64.5 & 4277 & 7306.6 & 5791.8 & 300.0 & 76.0 & - & - & OOM \\
\hline 70m_3_70 & 2098 & 53474.1 & 27786.1 & 1780 & 21424.2 & 11602.1 & 300.0 & 58.2 & 4947 & 3678.0 & 4312.5 & 300.0 & 84.5 & - & - & OOM \\
\hline 701_3_70 & 2080 & 69566.2 & 35823.1 & 1898 & 32793.6 & 17345.8 & 300.0 & 51.6 & 5844 & 6006.1 & 5925.0 & 300.0 & 83.5 & - & - & OOM \\
\hline 70s_5_30 & 1189 & 34016.0 & 17602.5 & 1173 & 11377.2 & 6275.1 & 300.0 & 64.4 & 3019 & 2394.4 & 2706.7 & 300.0 & 84.6 & - & - & OOM \\
\hline
\end{tabular}

(continued on next page) 
Table 7: Computational results of Dispatching rules, Sequential and Integrated heuristics (continued)

\begin{tabular}{|c|c|c|c|c|c|c|c|c|c|c|c|c|c|c|c|c|}
\hline \multirow[b]{2}{*}{ Instance } & \multicolumn{3}{|c|}{ Dispatching rules } & \multicolumn{5}{|c|}{ Sequential } & \multicolumn{6}{|c|}{ Integrated } & \multicolumn{2}{|c|}{ Best MIP bounds } \\
\hline & $E_{L A}$ & $E_{C S}$ & $E_{\tau}$ & $E_{L A}$ & $E_{C S}$ & $E_{\tau}$ & сpu & G\% & $E_{L A}$ & $E_{C S}$ & $E_{\tau}$ & сри & $\mathrm{G} \%$ & gap & UB & LB \\
\hline 70m_5_30 & 624 & 73034.1 & 36829.1 & 623 & 18676.8 & 9649.9 & 300.0 & 73.8 & 4391 & 3693.4 & 4042.2 & 300.0 & 89.0 & - & - & OOM \\
\hline 701_5_30 & 414 & 65472.1 & 32943.0 & 414 & 23484.7 & 11949.3 & 300.0 & 63.7 & 3336 & 5458.1 & 4397.0 & 300.0 & 86.7 & - & - & $\mathrm{OOM}$ \\
\hline 70 s_5_50 & 2189 & 41795.0 & 21992.0 & 2255 & 13117.9 & 7686.4 & 300.0 & 65.0 & 4696 & 3542.3 & 4119.2 & 300.0 & 81.3 & - & - & OOM \\
\hline 70m_5_50 & 850 & 53458.1 & 27154.1 & 850 & 17077.1 & 8963.5 & 300.0 & 67.0 & 5336 & 4445.2 & 4890.6 & 300.0 & 82.0 & - & - & OOM \\
\hline 701_5_50 & 739 & 58429.7 & 29584.4 & 739 & 19174.3 & 9956.7 & 300.0 & 66.3 & 5270 & 5840.9 & 5555.4 & 300.0 & 81.2 & - & - & $\mathrm{OOM}$ \\
\hline 70s_5_70 & 3404 & 40241.7 & 21822.9 & 2946 & 16937.8 & 9941.9 & 300.0 & 54.4 & 5891 & 2974.5 & 4432.7 & 300.0 & 79.7 & - & - & $\mathrm{OOM}$ \\
\hline 70m_5_70 & 2901 & 62528.1 & 32714.6 & 2655 & 25866.7 & 14260.8 & 300.0 & 56.4 & 7327 & 8793.2 & 8060.1 & 300.0 & 75.4 & - & - & OOM \\
\hline 701_5_70 & 1332 & 72718.7 & 37025.4 & 1190 & 31762.4 & 16476.2 & 300.0 & 55.5 & 6893 & 7988.2 & 7440.6 & 300.0 & 79.9 & - & - & OOM \\
\hline
\end{tabular}



increase is large.

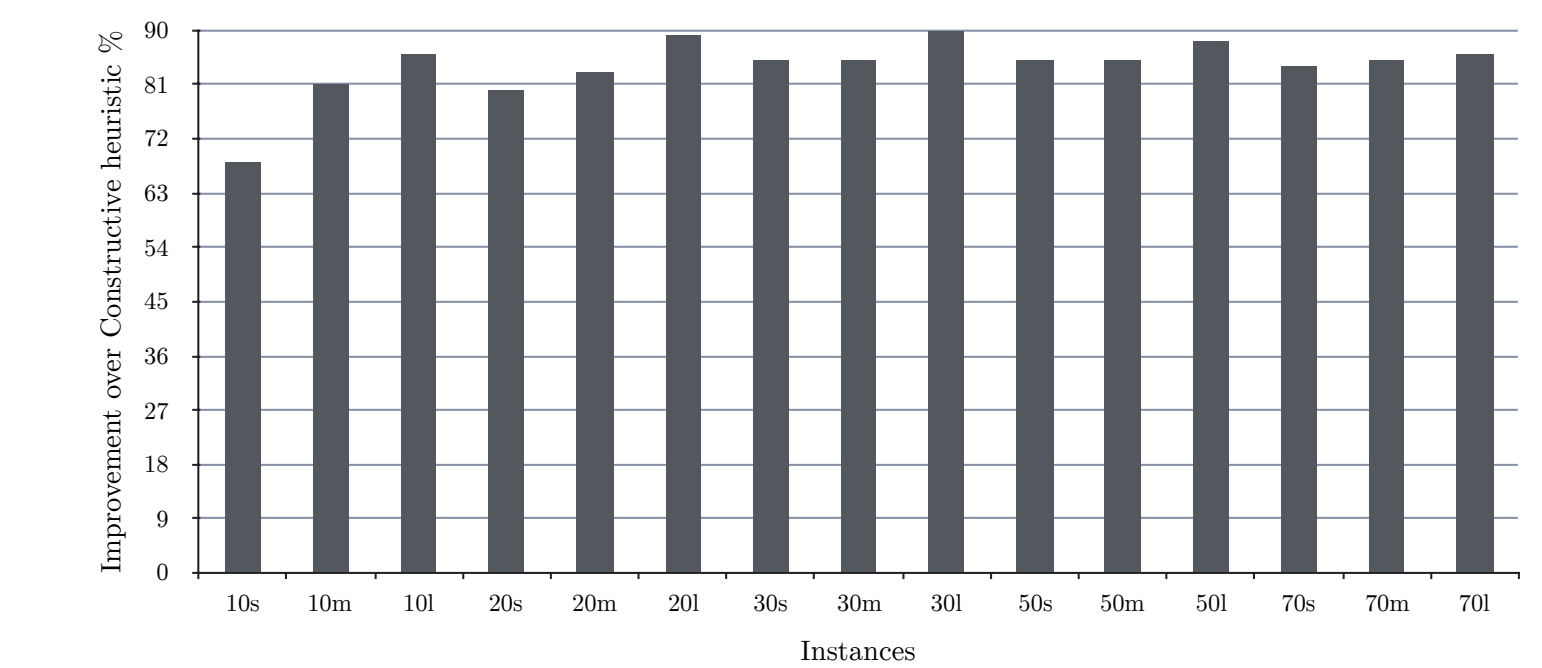

Figure 9: Comparison of the constructive and integrated heuristic's performance

Figure 9 illustrates how the integrated heuristic may improve over the constructive heuristic. The instances are grouped by their number of requests and storage sizes. The horizontal axis represents the instance categories while the vertical axis corresponds to the improvement over constructive heuristics. The results are averaged over each category and demonstrate that the improvement over the constructive heuristic is, on average, $84 \%$ over all instances. Given that the constructive heuristic reflects common operational dispatching rules, the potential performance

\subsection{Weight parameters and instance analysis}

Section 5.2 already briefly touched on the impact of the weight parameters $\alpha$ and $\beta$. In this section, the effect of adjusting these weight parameters is further investigated. Both a small $\left(10 s_{1} 1 \_70\right)$ and large $\left(50 m_{-} 1_{-} 50\right)$ instance were selected to illustrate the weight parameters' effect. Figure 10 presents the impact of adjusting $\alpha$ and $\beta$ for both the small (Figure 10(a), solved by $\mathcal{F}_{L A}+$ $\mathcal{F}_{C S}$ and $\mathcal{F}_{C W S}$ ), and the large instance (Figure 10(b)p, solved by the sequential and integrated heuristic. The figure illustrates the LAP $\left(E_{L A}\right), \operatorname{CSP}\left(E_{C S}\right)$ and weighted objective function $\left(E_{\tau}\right)$ values obtained by the integrated approach as well as the $E_{\tau}$ obtained by the sequential approach.

The weight parameters in the sequential approach do not impact upon the optimization process as the two sub-problems are solved separately. Sequential $E_{L A}$ and $E_{C S}$ are constant for all values of $\alpha$ and $\beta$. Therefore $E_{\tau}(\alpha, \beta)$ is a linear function.

As illustrated for $\frac{\beta}{\alpha}=0$ :

$$
\text { integrated } E_{\tau}=\text { sequential } E_{\tau}=\text { integrated } E_{L A}=\text { sequential } E_{L A}
$$

56 By increasing $\frac{\beta}{\alpha}$, the integrated $E_{L A}$ increases while the integrated $E_{C S}$ decreases. Interestingly the integrated $E_{\tau}$ is always lower than the sequential $E_{\tau}$ and by increasing $\frac{\beta}{\alpha}$ the gap between the integrated and sequential $E_{\tau}$ increases. 


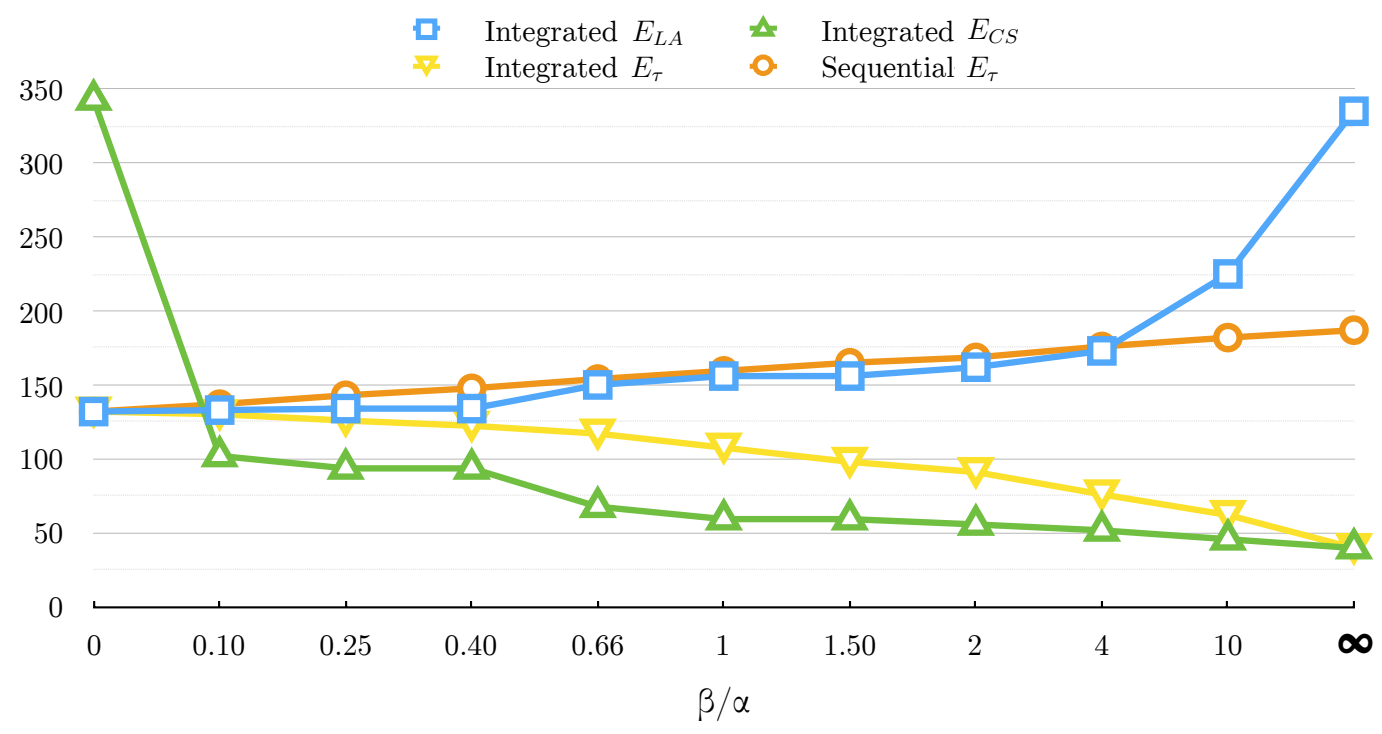

(a) Instance 10s_1_70 solved by the mathematical formulation

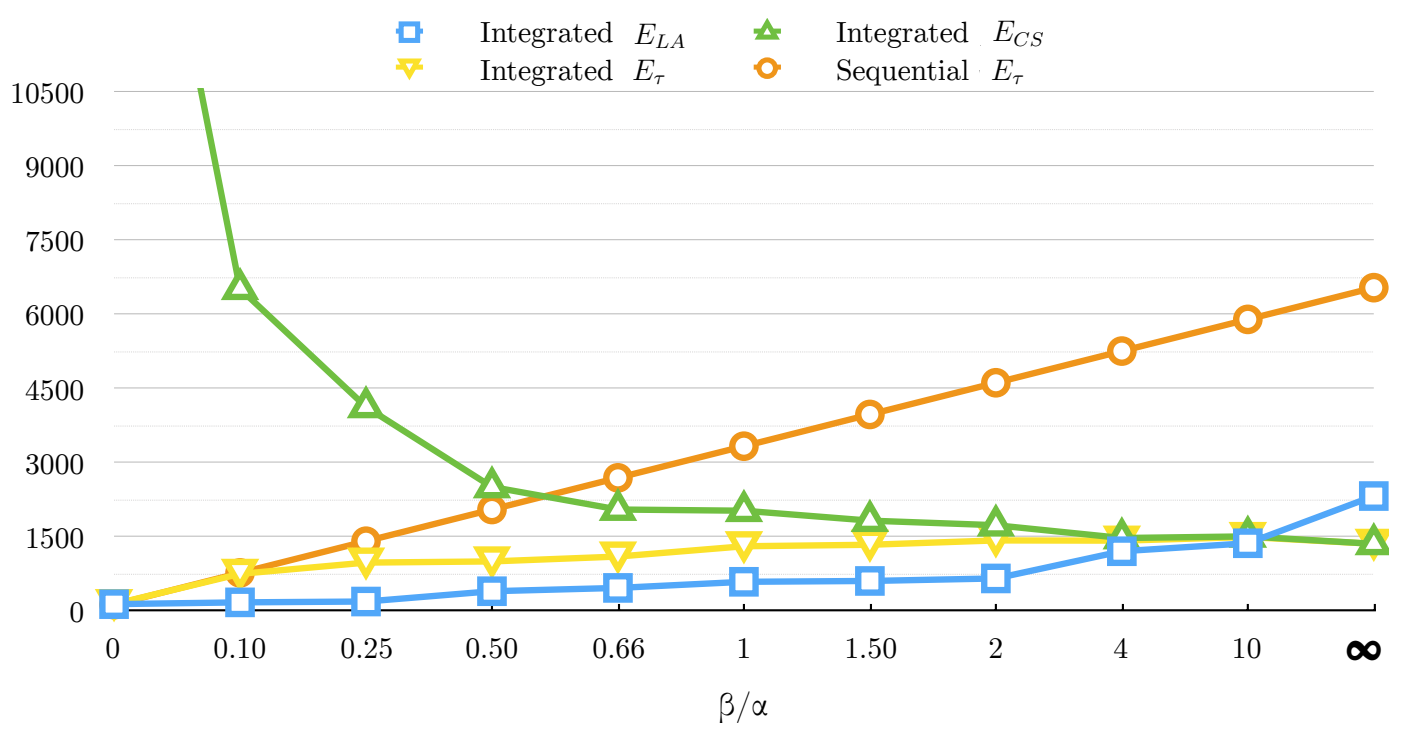

(b) Instance 50m_1_50 solved by the heuristic

Figure 10: Impact of the weight parameters' ratio.

Figure 11 demonstrates how different attributes of instances may impact the improvement of the sequential and integrated heuristic over the constructive heuristic. Figure 11(a) represents the average $E_{\tau}$ obtained by the constructive, sequential and integrated heuristic with respect to the stacking level. By increasing the maximum stacking level, the relative improvement over the solution obtained by the constructive heuristic decreases. By increasing the storage size from small to large (Figure 11(b)], the sequential and integrated heuristics improve the initial solution from $61 \%$ to $68 \%$ and from $80 \%$ to $87 \%$ respectively. Figure 11(d), meanwhile, illustrates how the load factor does not significantly influence the algorithms' improvement over the initial solution. 


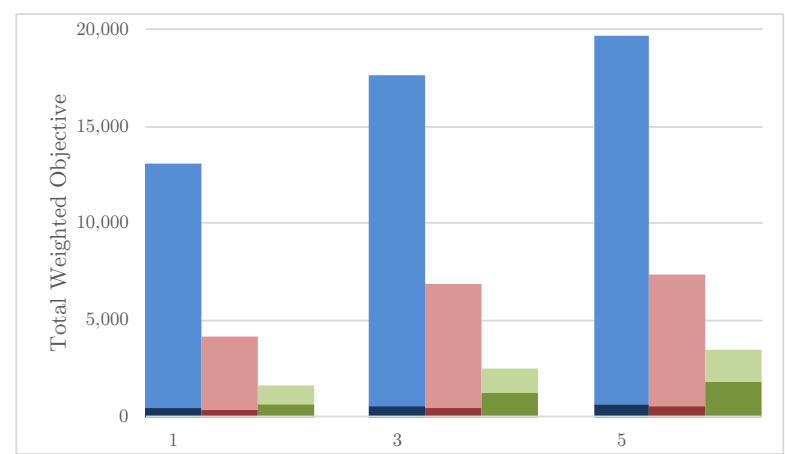

(a) Averaged cost over stacking level

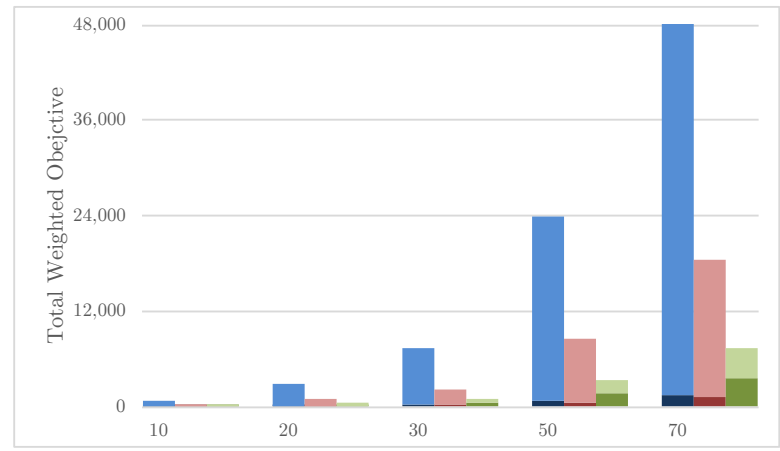

(c) Averaged cost over number of requests

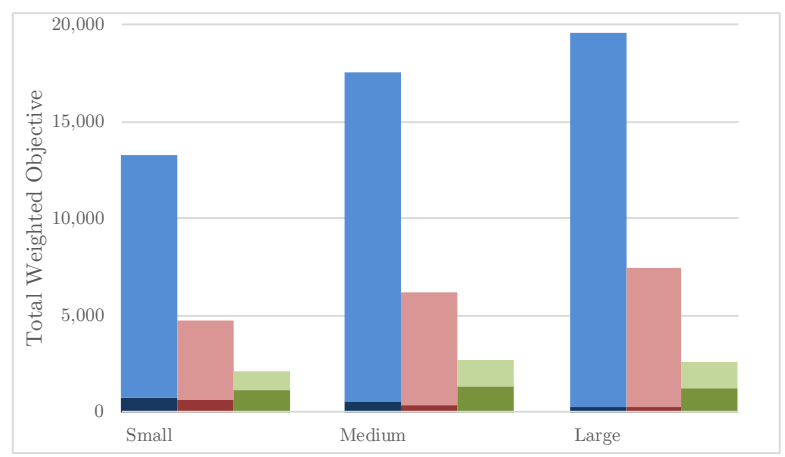

(b) Averaged cost over storage size

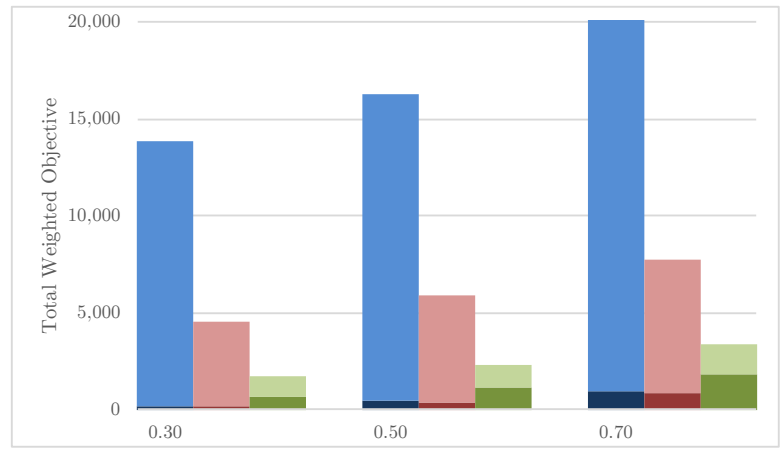

(d) Averaged cost over load factor

- Constructive Heuristic LAP a Sequential Heuristic LAP - Integrated Heuristic LAP

Figure 11: Instance attributes analysis by the constructive, sequential and integrated heuristic when $\alpha=\beta$.

\section{Conclusion}

This paper investigated the impact of integrating a location assignment problem (LAP) and a crane scheduling problem (CSP) in crane-operated warehouses by introducing the integrated Crane-operated Warehouse Scheduling Problem (CWSP). The CWSP assigns a storage location to input requests, assigns a crane to execute each request and decides how the set of requests must be sequenced per crane in such a way that the total storage cost and tardiness is minimized. Mixed Integer Programming (MIP) formulations for the LAP and CSP were presented in addition to a continuous-time MIP formulation which integrates both the LAP and CSP. This model considers realistic crane interactions in the storage area where cranes cannot pass each other and must keep a safety distance. Furthermore, a meta-heuristic based on Late Acceptance Hill Climbing (LAHC) was developed to overcome the limited scaling ability of solving the mathematical formulations by MIP solvers. In addition, 135 instances with various problem specifications were generated to enable validation and encourage future research.

A comprehensive computational study revealed how integrating the LAP and the CSP may lead to $48 \%$ improvement for the CSP while keeping the same level of quality for the LAP solutions. Subsequently, the results showed that by integrating the LAP and CSP into one problem, there is a significant reduction in the total weighted objective of $62 \%$. Furthermore, the benefit of the 
integrated heuristic over the MIP formulation was shown, where better solutions for both medium and large size instances were obtained compared to solving the MIP formulation on the respective instances. Additionally, a simulation of a real-world automated warehouse shows a significant potential for minimizing the storage cost and tardiness of the requests, when comparing the new procedures with typical dispatching rules.

In conclusion, integrating location assignment and crane scheduling coordinates the resources in automated warehouses or container terminals more effectively and eventually leads to efficiently storing the products or containers in the storage areas as well as minimizing the tardiness of the input and output requests.

Several interesting avenues exist to build upon this study in future research. Further solution approaches may be considered and investigated. Particularly, exact solution approaches would be a valuable contribution which may include proposing efficient lower bounding methods. Due to the nature of operations in automated warehouses, other research directions may focus on exploring the development of robust scheduling models which consider uncertain requests' arrival times.

\section{Appendix A. Detailed computational results}

Table A.8 presents detailed computational results obtained by the mathematical formulations. $\operatorname{gap}_{l a} \%$ (location assignment gap) and $g a p_{c s} \%$ (crane scheduling gap) indicate the gap obtained by $\mathcal{F}_{L A}$ and $\mathcal{F}_{C S}$, respectively, when solving the problem sequentially. gap $\%$ denotes the gap obtained by $\mathcal{F}_{A W S}$. 
Table A.8: Detailed computational results obtained by mathematical formulations

\begin{tabular}{|c|c|c|c|c|c|c|c|c|c|c|c|c|c|c|c|c|c|}
\hline \multirow[b]{2}{*}{ Ins. } & \multicolumn{7}{|c|}{$\mathcal{F}_{L A}+\mathcal{F}_{C S}$} & \multicolumn{5}{|c|}{$\mathcal{F}_{C W S}(\alpha>>\beta)$} & \multicolumn{5}{|c|}{$\mathcal{F}_{C W S}(\alpha=\beta)$} \\
\hline & $E_{L A}$ & $E_{C S}$ & $E_{\tau}(\alpha>>\beta)$ & $E_{\tau}(\alpha=\beta)$ & $g a p_{l a} \%$ & $g a p_{c s} \%$ & сpu & $E_{L A}$ & $E_{C S}$ & $E_{\tau}$ & gap\% & сpu & $E_{L A}$ & $E_{C S}$ & $E_{\tau}$ & gap\% & cpu \\
\hline 10s_1_30 & 0 & 267.03 & $2.670 \mathrm{E}+02$ & 133.51 & 0.00 & 0.00 & 107.28 & 0 & 55.13 & $5.513 \mathrm{E}+01$ & 0.00 & 49.79 & 1 & 51.53 & 26.26 & 0.00 & 170.92 \\
\hline 10m_1_30 & 0 & 478.41 & $4.784 \mathrm{E}+02$ & 239.20 & 0.00 & 0.00 & 604.05 & 0 & 37.62 & $3.762 \mathrm{E}+01$ & 0.00 & 60.78 & 0 & 37.62 & 18.81 & 0.00 & 513.46 \\
\hline 101_1_30 & 0 & 515.69 & $5.157 \mathrm{E}+02$ & 257.84 & 0.00 & 0.00 & 302.12 & 0 & 76.82 & $7.682 \mathrm{E}+01$ & 0.00 & 195.32 & 0 & 76.82 & 38.41 & 0.00 & 2044.59 \\
\hline $10 \mathrm{~s} \_1 \_50$ & 0 & 229.25 & $2.293 \mathrm{E}+02$ & 114.63 & 0.00 & 0.00 & 67.69 & 0 & 123.93 & $1.239 \mathrm{E}+02$ & 0.00 & 311.87 & 11 & 78.92 & 44.96 & 0.00 & 1009.35 \\
\hline 10m_1_50 & 0 & 219.18 & $2.192 \mathrm{E}+02$ & 109.59 & 0.00 & 0.00 & 360.00 & 0 & 56.45 & $5.645 \mathrm{E}+01$ & 0.00 & 91.66 & 7 & 40.07 & 23.53 & 0.00 & 475.39 \\
\hline 101_1_50 & 0 & 447.85 & $4.479 \mathrm{E}+02$ & 223.92 & 0.00 & 0.00 & 164.24 & 0 & 44.49 & $4.449 \mathrm{E}+01$ & 0.00 & 97.42 & 4 & 32.49 & 18.24 & 0.00 & 187.29 \\
\hline $10 s \_1 \_70$ & 132 & 186.97 & $1.320 \mathrm{E}+07$ & 159.49 & 0.00 & 0.00 & 115.83 & 132 & 135.97 & $1.320 \mathrm{E}+07$ & 0.00 & 11.06 & 156 & 59.25 & 107.62 & 0.00 & 1227.04 \\
\hline 10m_1_70 & 48 & 348.86 & $4.800 \mathrm{E}+06$ & 198.43 & 0.00 & 0.00 & 121.24 & 48 & 449.55 & $4.800 \mathrm{E}+06$ & 0.00 & 11.09 & 86 & 185.92 & 135.96 & 0.00 & 3290.77 \\
\hline 101_1_70 & 11 & 271.44 & $1.100 \mathrm{E}+06$ & 141.22 & 0.00 & 0.00 & 155.79 & 11 & 148.17 & $1.100 \mathrm{E}+06$ & 0.00 & 27.31 & 25 & 73.78 & 49.39 & 0.00 & 613.51 \\
\hline 10 s_3_30 & 13 & 352.10 & $1.300 \mathrm{E}+06$ & 182.55 & 0.00 & 0.00 & 442.41 & 13 & 179.72 & $1.300 \mathrm{E}+06$ & 0.00 & 119.08 & 49 & 76.07 & 62.53 & 35.51 & 3600.00 \\
\hline 10m_3_30 & 1 & 354.66 & $1.004 \mathrm{E}+05$ & 177.83 & 0.00 & 0.00 & 1254.72 & 1 & 107.51 & $1.001 \mathrm{E}+05$ & 0.00 & 3600.00 & 9 & 70.37 & 39.68 & 80.35 & 3600.00 \\
\hline 101_3_30 & 0 & 295.28 & $2.953 \mathrm{E}+02$ & 147.64 & 0.00 & 0.00 & 746.32 & 0 & 79.51 & $7.951 \mathrm{E}+01$ & 0.00 & 2387.71 & - & - & - & - & 3600.00 \\
\hline 10 s_3_50 & 53 & 317.77 & $5.300 \mathrm{E}+06$ & 185.39 & 0.00 & 0.00 & 879.75 & 53 & 201.77 & $5.300 \mathrm{E}+06$ & 0.00 & 3535.55 & 64 & 109.83 & 86.91 & 49.46 & 3600.00 \\
\hline 10m_3_50 & 31 & 409.77 & $3.100 \mathrm{E}+06$ & 220.39 & 0.00 & 0.00 & 911.50 & 31 & 332.35 & $3.100 \mathrm{E}+06$ & 0.00 & 303.92 & 41 & 126.77 & 83.88 & 80.67 & 3600.00 \\
\hline 101_3_50 & 9 & 454.43 & $9.005 \mathrm{E}+05$ & 231.72 & 0.00 & 0.00 & 1056.12 & 9 & 145.71 & $9.001 \mathrm{E}+05$ & 0.00 & 467.58 & 12 & 85.91 & 48.95 & 0.00 & 2366.16 \\
\hline 10 s_3_70 & 130 & 392.07 & $1.300 \mathrm{E}+07$ & 187.73 & 0.00 & 0.00 & 187.72 & 130 & 279.13 & $1.300 \mathrm{E}+07$ & 0.00 & 217.53 & 190 & 102.40 & 146.20 & 38.42 & 3600.00 \\
\hline 10m_3_70 & 64 & 433.66 & $6.400 \mathrm{E}+06$ & 248.83 & 0.00 & 0.00 & 212.35 & 64 & 492.57 & $6.400 \mathrm{E}+06$ & 0.00 & 92.46 & 93 & 77.90 & 85.45 & 20.80 & 3600.00 \\
\hline 101_3_70 & 98 & 441.02 & $9.800 \mathrm{E}+06$ & 269.51 & 0.00 & 0.00 & 574.97 & 98 & 566.37 & $9.800 \mathrm{E}+06$ & 0.00 & 502.12 & 134 & 118.11 & 126.05 & 55.16 & 3600.00 \\
\hline $10 s \_5 \_30$ & 18 & 322.97 & $1.800 \mathrm{E}+06$ & 170.48 & 0.00 & 0.00 & 2714.13 & 18 & 182.53 & $1.800 \mathrm{E}+06$ & 0.00 & 3600.00 & 35 & 93.23 & 64.11 & 66.30 & 3600.00 \\
\hline 10m_5_30 & 653 & 477.18 & $6.530 \mathrm{E}+07$ & 565.09 & 100.00 & 0.00 & 3224.10 & 9 & 275.46 & $9.002 \mathrm{E}+05$ & 0.01 & 3600.00 & 24 & 76.36 & 50.18 & 78.51 & 3600.00 \\
\hline 101_5_30 & 464 & 457.64 & $4.640 \mathrm{E}+07$ & 460.82 & 100.00 & 0.00 & 2845.26 & 6 & 244.62 & $6.002 \mathrm{E}+05$ & 0.00 & 3600.00 & 16 & 206.88 & 111.44 & 85.09 & 3600.00 \\
\hline 10 s_5_50 & 66 & 435.57 & $6.600 \mathrm{E}+06$ & 250.79 & 0.00 & 0.00 & 2300.19 & 66 & 292.55 & $6.600 \mathrm{E}+06$ & 6.82 & 3600.00 & - & & & & 3600.00 \\
\hline 10m_5_50 & 25 & - & - & - & 0.00 & - & 3600.00 & 25 & 294.46 & $2.500 \mathrm{E}+06$ & 42.16 & 3600.00 & 42 & 196.59 & 119.30 & 86.90 & 3600.00 \\
\hline 101_5_50 & 13 & 602.07 & $1.301 \mathrm{E}+06$ & 307.54 & 0.00 & 0.00 & 2306.55 & 13 & 159.11 & $1.300 \mathrm{E}+06$ & 0.00 & 3391.92 & 21 & 82.93 & 51.96 & 56.24 & 3600.00 \\
\hline 10 s_5_70 & 83 & 247.93 & $8.300 \mathrm{E}+06$ & 165.64 & 0.00 & 0.00 & 197.83 & 83 & 247.93 & $8.300 \mathrm{E}+06$ & 0.00 & 176.25 & 100 & 91.85 & 95.92 & 32.34 & 3600.00 \\
\hline 10m_5_70 & 59 & 419.26 & $5.900 \mathrm{E}+06$ & 239.13 & 0.00 & 0.00 & 1313.89 & 59 & 263.06 & $5.900 \mathrm{E}+06$ & 0.00 & 3600.00 & 75 & 211.27 & 143.13 & 78.93 & 3600.00 \\
\hline 101_5_70 & 43 & 541.38 & $4.301 \mathrm{E}+06$ & 292.19 & 0.00 & 0.00 & 1755.06 & 43 & 418.37 & $4.300 \mathrm{E}+06$ & 0.00 & 1223.19 & 51 & 87.02 & 69.01 & 29.27 & 3600.00 \\
\hline 20 s_1_30 & 0 & 1221.04 & $1.221 \mathrm{E}+03$ & 610.52 & 0.00 & 0.00 & 1970.28 & 0 & 160.38 & $1.603 \mathrm{E}+02$ & 35.40 & 3600.00 & - & - & - & & 3600.00 \\
\hline 20m_1_30 & 0 & 1821.80 & $1.822 \mathrm{E}+03$ & 910.90 & 0.00 & 0.00 & 2098.25 & 0 & 225.29 & $2.252 \mathrm{E}+02$ & 37.60 & 3600.00 & 1 & 235.90 & 118.45 & 84.91 & 3600.00 \\
\hline 201_1_30 & 0 & 2171.42 & $2.171 \mathrm{E}+03$ & 1085.71 & 0.00 & 0.00 & 2425.32 & 0 & 198.40 & $1.984 \mathrm{E}+02$ & 0.00 & 3420.09 & 0 & 261.18 & 130.59 & 75.71 & 3600.00 \\
\hline 20s_1_50 & 4 & 1412.59 & $4.014 \mathrm{E}+05$ & 708.30 & 0.00 & 0.00 & 1898.00 & 4 & 425.09 & $4.004 \mathrm{E}+02$ & 0.09 & 3600.00 & - & - & - & - & 3600.00 \\
\hline 20m_1_50 & 0 & 1373.24 & $1.373 \mathrm{E}+03$ & 686.21 & 0.00 & 0.00 & 1967.59 & 0 & 445.37 & $4.453 \mathrm{E}+02$ & 85.75 & 3600.00 & 9 & 351.42 & 180.21 & 90.47 & 3600.00 \\
\hline 201_1_50 & 0 & 1851.12 & $1.851 \mathrm{E}+03$ & 925.56 & 0.00 & 0.00 & 2205.82 & 0 & 155.85 & $1.558 \mathrm{E}+02$ & 0.00 & 3600.00 & 8 & 118.23 & 63.11 & 93.63 & 3600.00 \\
\hline $20 s \_1 \_70$ & 448 & 801.34 & $4.480 \mathrm{E}+07$ & 624.67 & 0.00 & 0.00 & 2062.93 & 448 & 692.26 & $4.480 \mathrm{E}+07$ & 0.00 & 2151.22 & 480 & 196.16 & 338.08 & 30.03 & 3600.00 \\
\hline 20m_1_70 & 241 & 2396.80 & $2.410 \mathrm{E}+07$ & 1318.90 & 0.00 & 0.00 & 1948.97 & 241 & 1287.71 & $2.410 \mathrm{E}+07$ & 0.00 & 1295.57 & 333 & 640.11 & 486.55 & 69.77 & 3600.00 \\
\hline 201_1_70 & 77 & 1210.96 & $7.701 \mathrm{E}+06$ & 643.98 & 0.00 & 0.00 & 2020.08 & 77 & 758.37 & $7.700 \mathrm{E}+06$ & 0.00 & 358.51 & 88 & 337.18 & 212.59 & 69.57 & 3600.00 \\
\hline 20 s_3_30 & 54 & 1328.04 & $5.401 \mathrm{E}+06$ & 691.02 & 77.77 & 80.41 & 3600.00 & - & - & - & - & 3600.00 & - & - & - & - & 3600.00 \\
\hline 20m_3_30 & 995 & 2031.35 & $9.950 \mathrm{E}+07$ & 1513.18 & 100.00 & 60.03 & 3600.00 & 15 & 740.32 & $1.500 \mathrm{E}+06$ & 0.04 & 3600.00 & 64 & 676.63 & 370.31 & 94.94 & 3600.00 \\
\hline 201_3_30 & 648 & & & & 100.00 & & 3600.00 & 0 & 277.50 & $2.775 \mathrm{E}+02$ & 47.91 & 3600.00 & 37 & 417.42 & 227.21 & 96.77 & 3600.00 \\
\hline $20 s \_3 \_50$ & 168 & 1434.26 & $1.680 \mathrm{E}+07$ & 799.13 & 58.53 & 73.01 & 3600.00 & - & - & - & - & 3600.00 & - & - & - & - & 3600.00 \\
\hline 20m_3_50 & 99 & 2203.80 & $9.902 \mathrm{E}+06$ & 1151.40 & 82.82 & 87.74 & 3600.00 & - & - & - & - & 3600.00 & 181 & 366.17 & 273.58 & 88.77 & 3600.00 \\
\hline 201_3_50 & 1344 & 1852.22 & $1.344 \mathrm{E}+08$ & 1598.11 & 99.62 & 49.66 & 3600.00 & 45 & 1123.48 & $4.501 \mathrm{E}+06$ & 66.67 & 3600.00 & 49 & 578.48 & 313.74 & 90.35 & 3600.00 \\
\hline
\end{tabular}


Table A.8: Detailed computational results obtained by mathematical formulations (continued)

\begin{tabular}{|c|c|c|c|c|c|c|c|c|c|c|c|c|c|c|c|c|c|}
\hline \multirow{2}{*}{ Ins. } & \multicolumn{7}{|c|}{$\mathcal{F}_{L A}+\mathcal{F}_{C S}$} & \multicolumn{5}{|c|}{$\mathcal{F}_{C W S}(\alpha>>\beta)$} & \multicolumn{5}{|c|}{$\mathcal{F}_{C W S}(\alpha=\beta)$} \\
\hline & $E_{L A}$ & $E_{C S}$ & $E_{\tau}(\alpha>>\beta)$ & $E_{\tau}(\alpha=\beta)$ & $\operatorname{gap}_{l a} \%$ & $g a p_{c s} \%$ & сри & $E_{L A}$ & $E_{C S}$ & $E_{\tau}$ & gap $\%$ & cpu & $E_{L A}$ & $E_{C S}$ & $E_{\tau}$ & gap\% & cpu \\
\hline 20 s_3_70 & 423 & 1552.35 & $4.230 \mathrm{E}+07$ & 987.67 & 14.42 & 59.01 & 3600.00 & 424 & 2902.26 & $4.240 \mathrm{E}+07$ & 14.62 & 3600.00 & 528 & 390.82 & 459.41 & 55.85 & 3600.00 \\
\hline 20m_3_70 & 168 & 1491.69 & $1.680 \mathrm{E}+07$ & 829.84 & 0.00 & 76.80 & 3600.00 & 168 & 1407.24 & $1.680 \mathrm{E}+07$ & 11.31 & 3600.00 & - & - & - & - & 3600.00 \\
\hline 201_3_70 & 274 & 2989.96 & $2.740 \mathrm{E}+07$ & 1631.98 & 44.16 & 81.51 & 3600.00 & - & & & - & 3600.00 & - & - & - & - & 3600.00 \\
\hline 20s_5_30 & 923 & 939.96 & $9.230 \mathrm{E}+07$ & 931.48 & 99.89 & 42.39 & 3600.00 & 68 & 1630.26 & $6.801 \mathrm{E}+06$ & 95.45 & 3600.00 & - & - & - & - & 3600.00 \\
\hline 20m_5_30 & 886 & - & & & 100.00 & & 3600.00 & 43 & 2017.09 & $4.302 \mathrm{E}+06$ & 98.66 & 3600.00 & - & - & - & - & 3600.00 \\
\hline $20 s \_5 \_50$ & 1527 & 1051.42 & $1.527 \mathrm{E}+08$ & 1289.21 & 99.21 & 34.04 & 3600.00 & - & - & - & - & 3600.00 & - & - & - & - & 3600.00 \\
\hline 20m_5_50 & 1450 & - & - & - & 99.72 & - & 3600.00 & 87 & 2062.80 & $8.702 \mathrm{E}+06$ & 88.24 & 3600.00 & - & - & - & - & 3600.00 \\
\hline 20s_5_70 & 330 & 1499.62 & $3.300 \mathrm{E}+07$ & 914.81 & 59.09 & 70.46 & 3600.00 & - & - & - & - & 3600.00 & - & - & - & - & 3600.00 \\
\hline 20m_5_70 & 249 & 1435.31 & $2.490 \mathrm{E}+07$ & 842.15 & 83.93 & 76.39 & 3600.00 & 385 & 1738.69 & $3.850 \mathrm{E}+07$ & 78.95 & 3600.00 & - & - & - & - & 3600.00 \\
\hline 201_5_70 & 129 & - & & - & 82.17 & - & 3600.00 & - & - & & - & 3600.00 & 158 & 812.23 & 485.11 & 89.34 & 3600.00 \\
\hline 30s_1_30 & 0 & 3495.86 & $3.496 \mathrm{E}+03$ & 1747.93 & 0.00 & 0.00 & 2214.11 & 0 & 668.80 & $6.688 \mathrm{E}+02$ & 91.89 & 3600.00 & - & - & - & - & 3600.00 \\
\hline 30m_1_30 & 0 & 4501.77 & $4.502 \mathrm{E}+03$ & 2250.88 & 0.00 & 0.00 & 2554.77 & 0 & 921.33 & $9.213 \mathrm{E}+02$ & 90.25 & 3600.00 & - & - & - & - & 3600.00 \\
\hline 301_1_30 & 0 & 4911.02 & $4.911 \mathrm{E}+03$ & 2455.51 & 0.00 & 94.73 & 3600.00 & 0 & 634.49 & $6.344 \mathrm{E}+02$ & 80.19 & 3600.00 & - & - & - & - & 3600.00 \\
\hline $30 \mathbf{s}_{1} 1 \_50$ & 98 & 3299.06 & $9.803 \mathrm{E}+06$ & 1698.53 & 0.00 & 0.00 & 3401.45 & 98 & 1303.66 & $9.801 \mathrm{E}+06$ & 11.74 & 3600.00 & 210 & 729.63 & 469.81 & 85.70 & 3600.00 \\
\hline 30m_1_50 & 19 & 3742.17 & $1.904 \mathrm{E}+06$ & 1880.58 & 100.00 & 95.28 & 3600.00 & 19 & 2616.36 & $1.902 \mathrm{E}+06$ & 99.99 & 3600.00 & 75 & 1880.72 & 977.86 & 97.79 & 3600.00 \\
\hline 301_1_50 & 0 & 4683.93 & $4.684 \mathrm{E}+03$ & 2737.80 & 0.00 & 0.00 & 2737.80 & 0 & 724.51 & $7.245 \mathrm{E}+02$ & 94.12 & 3600.00 & - & - & - & - & 3600.00 \\
\hline 30s_1_70 & 934 & 2416.98 & $9.340 \mathrm{E}+07$ & 1675.49 & 10.38 & 66.61 & 3600.00 & 937 & 2667.16 & $9.370 \mathrm{E}+07$ & 11.20 & 3600.00 & - & - & - & - & 3600.00 \\
\hline 30m_1_70 & 544 & 5919.16 & $5.441 \mathrm{E}+07$ & 3231.58 & 8.63 & 87.56 & 3600.00 & 554 & 3078.50 & $5.540 \mathrm{E}+07$ & 10.29 & 3600.00 & 631 & 2046.16 & 1338.58 & 78.02 & 3600.00 \\
\hline 301_1_70 & 249 & 3310.89 & $2.490 \mathrm{E}+07$ & 1779.94 & 0.00 & 0.00 & 2641.01 & - & - & - & - & 3600.00 & 268 & 1623.10 & 945.55 & 85.39 & 3600.00 \\
\hline 30s_3_30 & 1585 & 2858.33 & $1.585 \mathrm{E}+08$ & 2221.66 & 99.74 & 59.80 & 3600.00 & - & - & - & - & 3600.00 & - & - & - & - & 3600.00 \\
\hline 30 s_3_50 & 330 & 3643.15 & $3.300 \mathrm{E}+07$ & 1986.57 & 70.00 & 84.45 & 3600.00 & - & - & - & - & 3600.00 & - & - & - & - & 3600.00 \\
\hline 30s_3_70 & 821 & 3058.59 & $8.210 \mathrm{E}+07$ & 1939.79 & 18.27 & 69.32 & 3600.00 & 840 & 3613.93 & $8.400 \mathrm{E}+07$ & 19.35 & 3600.00 & - & - & - & - & 3600.00 \\
\hline 30m_3_70 & 371 & 3394.37 & $3.710 \mathrm{E}+07$ & 1882.68 & 39.35 & 84.73 & 3600.00 & - & - & - & - & 3600.00 & - & - & - & - & 3600.00 \\
\hline 301_3_70 & 2589 & & & & 97.29 & & 3600.00 & - & - & - & - & 3600.00 & 684 & 9339.08 & 5019.04 & 98.06 & 3600.00 \\
\hline 30s_5_70 & 756 & 4492.94 & $7.560 \mathrm{E}+07$ & 2624.47 & 85.58 & 80.18 & 3600.00 & - & - & - & - & 3600.00 & - & - & - & - & 3600.00 \\
\hline $50 s \_1 \_30$ & 0 & 16686.92 & $1.669 \mathrm{E}+04$ & 8343.46 & 0.00 & 0.00 & 2892.64 & - & - & - & - & 3600.00 & - & - & - & - & 3600.00 \\
\hline 50m_1_30 & 0 & 17645.35 & $1.765 \mathrm{E}+04$ & 8822.67 & 0.00 & 97.98 & 3600.00 & 0 & 8264.20 & $8.264 \mathrm{E}+03$ & 98.38 & 3600.00 & - & - & - & - & 3600.00 \\
\hline 50s_1_50 & 401 & 12290.55 & $4.011 \mathrm{E}+07$ & 6345.77 & 34.41 & 93.74 & 3600.00 & - & - & - & - & 3600.00 & - & - & - & - & 3600.00 \\
\hline 50m_1_50 & 250 & 13869.28 & $2.501 \mathrm{E}+07$ & 7059.64 & 84.40 & 95.04 & 3600.00 & - & - & - & - & 3600.00 & - & - & - & - & 3600.00 \\
\hline 501_1_50 & 11 & 15907.70 & $1.116 \mathrm{E}+06$ & 7959.35 & 90.90 & 97.80 & 3600.00 & - & - & - & - & 3600.00 & 146 & 9867.24 & 5006.62 & 98.98 & 3600.00 \\
\hline 50s_1_70 & 1743 & 11544.01 & $1.743 \mathrm{E}+08$ & 6643.50 & 16.40 & 83.19 & 3600.00 & 1772 & 6898.15 & $1.772 \mathrm{E}+08$ & 17.77 & 3600.00 & 2370 & 4006.59 & 3188.29 & 75.01 & 3600.00 \\
\hline 50m_1_70 & 1017 & 19637.64 & $1.017 \mathrm{E}+08$ & 10327.32 & 14.06 & 92.16 & 3600.00 & - & - & - & - & 3600.00 & - & - & - & - & 3600.00 \\
\hline 501_1_70 & 599 & 20988.08 & $5.992 \mathrm{E}+07$ & 10793.54 & 12.18 & 95.38 & 3600.00 & - & - & - & - & 3600.00 & - & - & - & - & 3600.00 \\
\hline 50s_3_70 & 3904 & 12233.50 & $3.904 \mathrm{E}+08$ & 8068.75 & 78.32 & 72.48 & 3600.00 & - & - & - & - & 3600.00 & - & - & - & - & 3600.00 \\
\hline 70s_1_30 & 67 & 19600.96 & $6.720 \mathrm{E}+06$ & 9833.98 & 100.00 & 96.20 & 3600.00 & - & - & - & - & 3600.00 & - & - & - & - & 3600.00 \\
\hline 70 s_1_50 & 1436 & 20060.26 & $1.436 \mathrm{E}+08$ & 10748.13 & 48.11 & 90.22 & 3600.00 & - & - & - & - & 3600.00 & - & - & - & - & 3600.00 \\
\hline 70s_1_70 & 3225 & 16797.21 & $3.225 \mathrm{E}+08$ & 10011.10 & 18.63 & 80.97 & 3600.00 & - & - & - & - & 3600.00 & - & - & - & - & 3600.00 \\
\hline 70m_1_70 & 2023 & 29211.91 & $2.023 \mathrm{E}+08$ & 15617.45 & 19.37 & 90.92 & 3600.00 & - & - & - & - & 3600.00 & - & - & - & - & 3600.00 \\
\hline 701_1_70 & 1332 & 41511.74 & $1.332 \mathrm{E}+08$ & 21421.87 & 20.79 & 95.56 & 3600.00 & - & - & - & - & 3600.00 & - & - & - & - & 3600.00 \\
\hline
\end{tabular}




\section{References}

Boysen, N., Emde, S., 2016. The parallel stack loading problem to minimize blockages. European Journal of Operational Research 249, 618-627.

Boysen, N., Stephan, K., 2016. A survey on single crane scheduling in automated storage/retrieval systems. European Journal of Operational Research 254, 691-704.

Burke, E.K., Bykov, Y., 2017. The late acceptance hill-climbing heuristic. European Journal of Operational Research $258,70-78$

Chen, L., Lu, Z., 2012. The storage location assignment problem for outbound containers in a maritime terminal. International Journal of Production Economics 135, 73-80.

Darvish, M., Coelho, L.C., 2018. Sequential versus integrated optimization: Production, location, inventory control, and distribution. European Journal of Operational Research 268, $203-214$

Dorndorf, U., Schneider, F., 2010. Scheduling automated triple cross-over stacking cranes in a container yard. OR Spectrum 32, 617-632.

Escudero, L., 1988. An inexact algorithm for the sequential ordering problem. European Journal of Operational Research 37, 236-249.

Gharehgozli, A.H., Laporte, G., Yu, Y., de Koster, R., 2015. Scheduling Twin Yard Cranes in a Container Block. Transportation Science 49, 686-705.

Gharehgozli, A.H., Vernooij, F.G., Zaerpour, N., 2017. A simulation study of the performance of twin automated stacking cranes at a seaport container terminal. European Journal of Operational Research 261, 108-128.

Gharehgozli, A.H., Yu, Y., de Koster, R., Udding, J.T., 2014. A decision-tree stacking heuristic minimising the expected number of reshuffles at a container terminal. International Journal of Production Research 52, 25922611.

Jovanovic, R., Voß, S., 2014. A chain heuristic for the blocks relocation problem. Computers \& Industrial Engineering $75,79-86$.

Ku, D., Arthanari, T.S., 2016. On the abstraction method for the container relocation problem. Computers \& Operations Research 68, 110-122.

Li, W., Goh, M., Wu, Y., Petering, M., de Souza, R., Wu, Y., 2012. A continuous time model for multiple yard crane scheduling with last minute job arrivals. International Journal of Production Economics 136, 332-343.

Li, W., Wu, Y., Petering, M., Goh, M., Souza, R.D., 2009. Discrete time model and algorithms for container yard crane scheduling. European Journal of Operational Research 198, 165-172.

López-Ibáñez, M., Dubois-Lacoste, J., Cáceres, L.P., Birattari, M., Stützle, T., 2016. The irace package: Iterated racing for automatic algorithm configuration. Operations Research Perspectives 3, 43-58.

Montemanni, R., Smith, D., Rizzoli, A., Gambardella, L., 2009. Sequential ordering problems for crane scheduling in port terminals. International Journal of Simulation and Process Modelling 5, 348-361. doi 10.1504/IJSPM.2009. 032597.

Park, T., Choe, R., Hun Kim, Y., Ryel Ryu, K., 2011. Dynamic adjustment of container stacking policy in an automated container terminal. International Journal of Production Economics 133, 385-392.

Wu, Y., Li, W., Petering, M.E.H., Goh, M., de Souza, R., 2015. Scheduling multiple yard cranes with crane interference and safety distance requirement. Transportation Science 49, 990-1005.

Zhang, C., Wu, T., Zhong, M., Zheng, L., Miao, L., 2014. Location assignment for outbound containers with adjusted weight proportion. Computers \& Operations Research 52, 84-93. 